Alcançabilidade e controlabilidade médias para sistemas lineares com saltos markovianos a tempo contínuo

Alfredo Rafael Roa Narváez 



\title{
Alcançabilidade e controlabilidade médias para sistemas lineares com saltos markovianos a tempo contínuo
}

\author{
Alfredo Rafael Roa Narváez \\ Orientador: Prof. Dr. Eduardo Fontoura Costa
}

Tese apresentada ao Instituto de Ciências Matemáticas e de Computação - ICMC-USP, como parte dos requisitos para obtenção do título de Doutor em Ciências - Ciências de Computação e Matemática Computacional. VERSÃO REVISADA. 
Ficha catalográfica elaborada pela Biblioteca Prof. Achille Bassi e Seção Técnica de Informática, ICMC/USP, com os dados fornecidos pelo(a) autor(a)

Roa Narváez, Alfredo Rafael
Alcançabilidade e controlabilidade médias para
Sistemas lineares com saltos markovianos a tempo
contínuo / Alfredo Rafael Roa Narváez; orientador
Eduardo Fontoura Costa. -- São Carlos, 2015.
$78 \mathrm{p}$.
Tese (Doutorado - Programa de Pós-Graduação em
Ciências de Computaça e Matemática Computacional) --
Instituto de Ciências Matemáticas e de Computação,
Universidade de São Paulo, 2015.
1. Alcançabilidade. 2. Controlabilidade. 3.
Sistemas lineares com saltos markovianos. I. Costa,
Eduardo Fontoura, orient. II. Título.




\section{Dedicatória}

Aos meus amados pais,

Yolima e Alfredo. 



\section{Agradecimentos}

Ao meu orientador Eduardo Fontoura Costa pela excelente orientação oferecida na realização deste trabalho e pela motivação, colaboração e apoio nos momentos difíceis.

Aos meus pais Alfredo Roa Gómez e Yolima Narváez Camargo e às minhas irmãs Karen Roa Narváez e Yolima Roa Narváez por todo amor, suporte, carinho e incentivo.

À minha esposa Jeinny pelo amor e compreensão e ao meu filho Alejandro pela grande motivação para prosseguir na culminação deste trabalho.

À Coordenação de Aperfeiçoamento de Pessoal de Nível Superior - CAPES, pela bolsa concedida a este trabalho.

A todos os colegas, professores e funcionários do Instituto de Ciências Matemáticas e de Computação da Universidade de São Paulo. 



\section{Resumo}

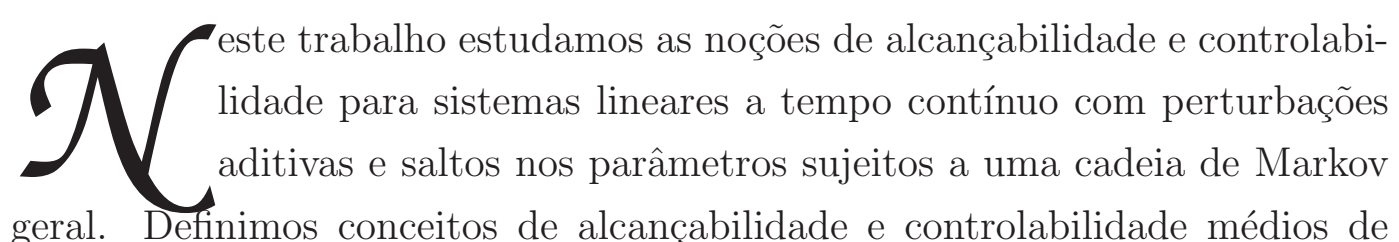

maneira natural exigindo que os valores esperados dos gramianos correspondentes sejam definidos positivos. Visando obter uma condição testável para ambos os conceitos, introduzimos conjuntos de matrizes de alcançabilidade e de controlabilidade para esta classe de sistemas e usamos certas propriedades de invariância para mostrar que: o sistema é alcançável em média, e, analogamente, controlável em média, se e somente se as matrizes respectivas, de alcançabilidade e de controlabilidade, têm posto completo. Usamos alcançabilidade média de sistemas para mostrar que a matriz de segundo momento do estado é definida positiva com uma margem uniforme. Uma consequência deste resultado no problema de estimação linear do estado é que a matriz de covariância do erro de estimação é positiva definida em média, no sentido que existe um nível mínimo de ruído nas estimativas. Na sequência, para estimadores lineares markovianos, estudamos a limitação do valor esperado da matriz de covariância do erro para mostrar que o filtro é estável num certo sentido, sendo esta uma propriedade desejável em aplicações reais. Quanto às aplicações da controlabilidade média, usamos este conceito para estabelecer condições necessárias e suficientes que garantem a existência de um processo de controle que leva a componente contínua do estado do sistema para a origem em tempo finito e com probabilidade positiva. 



\section{Abstract}

$\mathrm{n}$ this work we study the reachability and controllability notions for continuous-time linear systems with exogenous inputs and jump parameters driven by a quite general Markov chain. We define a rather natural average reachability and controllability concepts by requiring that the associated gramians are average positive definite, respectively. Aiming at testable conditions for each concept, we introduce certain sets of matrices linked with the gramians, and employ some invariance properties to find rank-based conditions. We show for average reachable systems that the state second moment is positive definite. One consequence of this result in the context of linear estimation for reachable systems is that the expectation of the error covariance matrix is positive definite. Moreover, for linear markovian filters we study the average boundedness of the error covariance matrix to show that the filter is stable in an appropriate sense, which consists in a property that is desirable in real applications. Regarding the average controllability concept, we show that it is a necessary and sufficient condition for the feasibility of the following control problem: find a control process that drives the continuous component of the state to zero in finite time with positive probability. 




\section{Sumário}

Agradecimentos

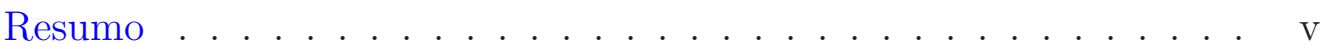

Abstract . . . . . . . . . . . . . . . . vii

1 Introdução 3

2 Notações e definições $\quad 9$

2.1 Sistemas lineares . . . . . . . . . . . . . . . . . . 9 9

2.2 Sistemas lineares com saltos markovianos . . . . . . . . . . . 13

3 Resultados preliminares $\quad 17$

3.1 Propriedades do gramiano de observabilidade de SLSM . . . . 17

3.2 Propriedades dos gramianos de alcançabilidade e controlabilidade de SLSM e relações de dualidade com a observabilidade . . 23

4 Alcançabilidade média e um teste computacional 29

4.1 Alcançabilidade média sujeita a $\pi \ldots$. . . . . . . . . . . 29

4.2 Alcançabilidade Média . . . . . . . . . . . . . . . . . . . . . 34

5 Consequências da alcançabilidade média 37

5.1 Positividade do segundo momento do estado . . . . . . . . . . . 38

5.2 Propriedades da covariância do erro de estimação de SLSM . . . 44

6 Controlabilidade média e propriedades $\quad 55$

6.1 Factibilidade no problema de controlabilidade . . . . . . . . 57

$\begin{array}{lll}7 & \text { Conclusões } & 61\end{array}$ 
A Observabilidade e construtibilidade para sistemas lineares

B Comparação de desempenho do ELQMM e o FKB para SLSM 67

Referências 


\section{Lista de Figuras}

1.1 Uma realização da trajetória $x(t)$ de um SLSM cuja dinâmica é modulada por realizações de uma cadeia de Markov $\{\theta(t)\}_{t \geq 0}$. . 4

4.1 Porção de trajetórias correspondentes a 1000 realizações para o processo $\{x(t)\}, t \geq 0$ do sistema no Exemplo 3. . . . . . . . 34

5.1 Menor autovalor de $X_{j}(t), j \in \mathcal{S}=\{1,2,3\}$, para o sistema no Exemplo 5(a). . . . . . . . . . . . . . . . 43

5.2 Menor autovalor de $X_{j}(t), j \in\{1,2\}$ para o sistema no Exemplo

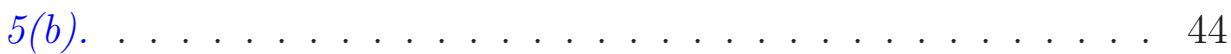

5.3 Menor autovalor de $\mathcal{E}\left\{x(t) x(t)^{\prime}\right\}, t \in[0,5]$, para o sistema no Exemplo 5(c). . . . . . . . . . . . . . . . 44

5.4 Norma das matrizes $\mathcal{E}\{P(t)\}$ para diferentes valores de $\Sigma$ (linhas sólidas) e $\mathcal{E}\{Q(t)\}$ (linha pontilhada) sobre o intervalo $[0,10]$ para o ELQMM do Exemplo 6. . . . . . . . . . . . . . 52

5.5 Norma da matriz $\mathcal{E}\{P(t)\}$ sobre o intervalo [0,30] para o ELQMM do Exemplo 7(a). . . . . . . . . . . . . . . . 53

5.6 Norma da matriz $\mathcal{E}\{P(t)\}$ (linha sólida) e $\mathcal{E}\{Q(t)\}$ (linha pontilhada) sobre o intervalo $[0,10]$ para o ELQMM do Exemplo r(b). . . . . . . . . . . . . . . . . 5 53

6.1 Três realizações diferentes de $x(t)$ para o sistema no Exemplo 12. 59 


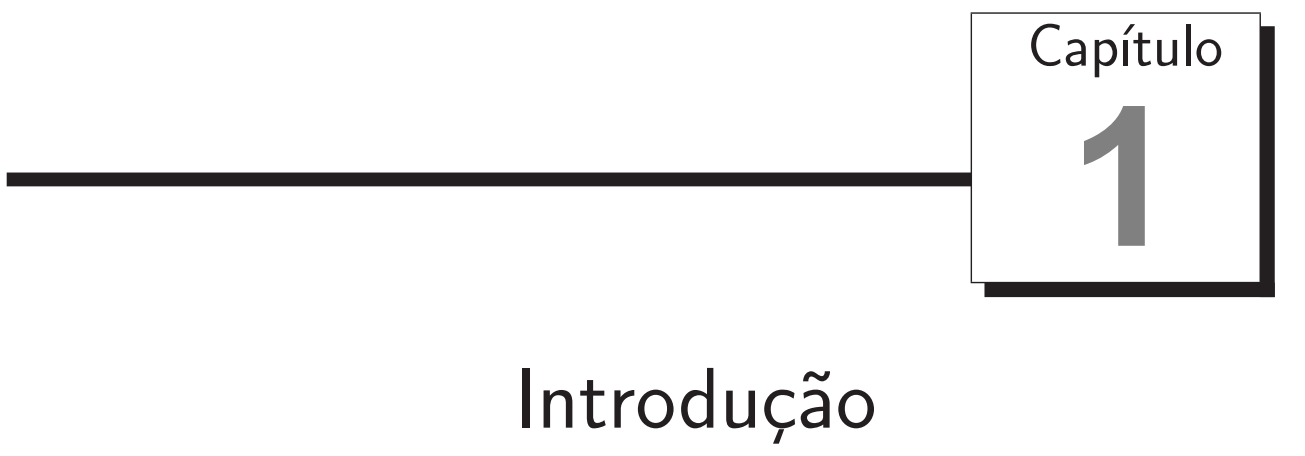

Em situações práticas, os sistemas dinâmicos são muitas vezes vulneráveis a mudanças abruptas em seu comportamento, por exemplo, falhas imprevisíveis em componentes ou perturbações repentinas do ambiente. Estes fenômenos são descritos frequentemente com processos que combinam dinâmicas contínuas com eventos discretos os quais são conhecidos na literatura como sistemas híbridos de chaveamento. Existe uma classe em particular deste tipo de sistemas nos que a lei de chaveamento é uma cadeia de Markov $\{\theta(t)\}_{t \geq 0}$ a tempo contínuo com espaço de estado finito. Os valores que toma $\theta(t)$ modulam a dinâmica da trajetória contínua $x(t)$ a qual é dada por sistemas de equações diferenciais lineares. Os sistemas híbridos de chaveamento desta classe são conhecidos como sistemas lineares com saltos markovianos (SLSM). Abordaremos neste trabalho os SLSM a tempo contínuo, denotando o estado destes sistemas pelo par $(x(t), \theta(t))$. Nos SLSM as matrizes coeficientes dos sistemas lineares dependem dos estados da cadeia de Markov, isto é, quando $\theta(t)$ toma um dos seus valores, digamos $\theta(t)=i$, a dinâmica do sistema está determinada por matrizes coeficientes associadas ao estado $i$, de forma que $x(t)$ evolui de acordo com esta dinâmica; após um certo tempo aleatório, $\theta(t)$ muda para um novo estado $j \neq i$, permanecendo ali até um novo salto ocorrer; desta forma, a dinâmica do sistema está determinada agora pelas matrizes coeficientes associadas ao estado $j$ e assim por diante. A Figura 1.1 ilustra um exemplo da evolução usual da trajetória $x(t)$ de um SLSM a qual muda abruptamente sua dinâmica de acordo com os estados 1,2 e 3 de uma cadeia de Markov. 


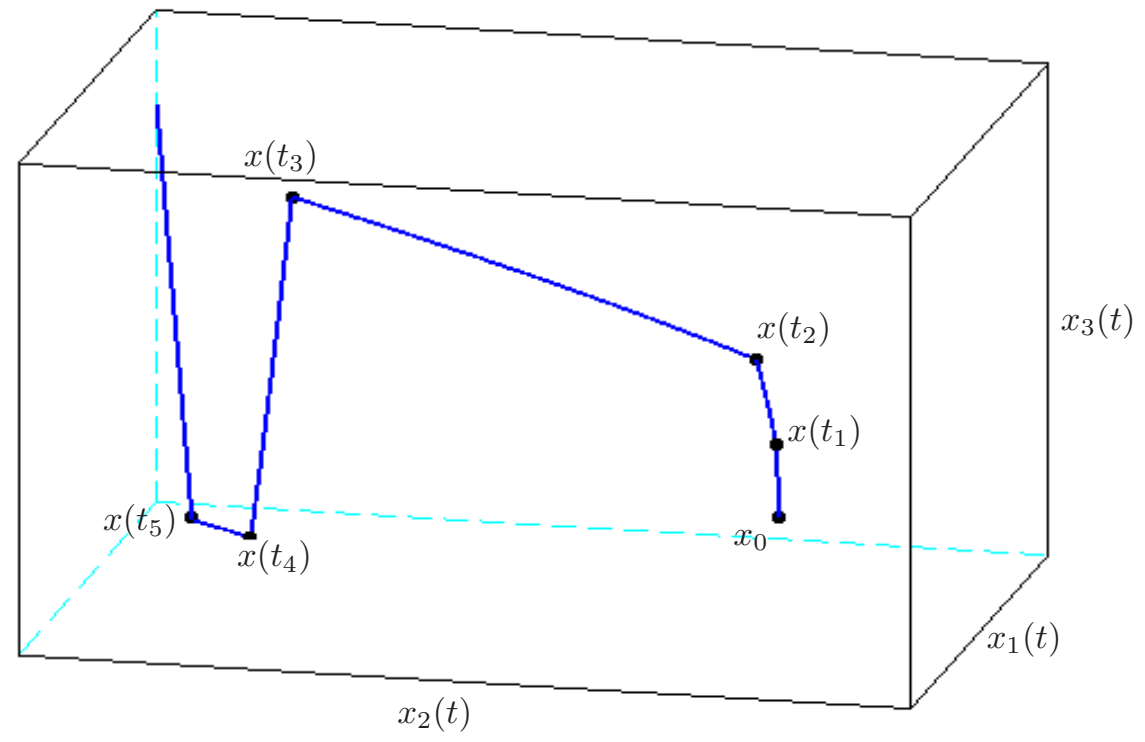

Figura 1.1: Uma realização da trajetória $x(t)$ de um SLSM cuja dinâmica é modulada por realizações de uma cadeia de Markov $\{\theta(t)\}_{t \geq 0}$.

Os SLSM generalizam os sistemas lineares e, ainda, são suficientemente especializados para apresentar resultados fortes que mimetizam propriedades estruturais de sistemas lineares. Devido a isto há uma grande motivação no estudo de SLSM e a pesquisa neste tipo de sistemas adquire uma relevância cada vez maior, dada a diversidade de temas a serem abordados, vide (Costa et al., 2005b, 2013; Dragan et al., 2013). Em particular, algumas propriedades estruturais de SLSM têm sido estudadas muito detalhadamente, tais como a controlabilidade estocástica e a estabilizabilidade as quais são apresentadas no artigo seminal (Ji e Chizeck, 1990) assim como nos trabalhos (Costa et al., 1999; Costa e Fragoso, 1995; Costa e Marques, 2000; Fragoso e Baczynski, 2001; Ji e Chizeck, 1992; Morozan, 1995; Costa et al., 2005b). Existe uma série de noções sob a denominação de conceitos fracos, como observabilidade fraca e detetabilidade fraca (Costa e do Val, 2002a,b; Costa et al., 2005a; do Val e Costa, 2002 , 2005), os quais envolvem funcionais de custo que podem ser expressados em termos da esperança do gramiano de observabilidade. O papel destes conceitos em controle é agora bem conhecido. Neste texto, chamamos estas noções de observabilidade média e detetabilidade média ${ }^{\mathrm{a}}$. Também, foram introdu-

aNós usamos o termo média ao invés de fraca já que é mais precisa em consideração à 
zidos recentemente uma condição para controlabilidade média assim como o conceito de estabilizabilidade média para SLSM a tempo discreto, vide (Costa et al., 2006). Inspirados pela noção de observabilidade média, introduzimos aqui algumas noções de alcançabilidade e controlabilidade médias, baseadas nos gramianos (vide por exemplo (Davis, 2002)), de alcançabilidade

$$
\Upsilon_{\mathrm{rch}}\left(t_{0}, t_{1}\right)=\int_{t_{0}}^{t_{1}} \Phi\left(t_{1}, \tau\right) B_{\theta(\tau)} B_{\theta(\tau)}^{\prime} \Phi\left(t_{1}, \tau\right)^{\prime} d \tau
$$

e de controlabilidade,

$$
\Upsilon_{\mathrm{cnt}}\left(t_{0}, t_{1}\right)=\int_{t_{0}}^{t_{1}} \Phi\left(t_{0}, \tau\right) B_{\theta(\tau)} B_{\theta(\tau)}^{\prime} \Phi\left(t_{0}, \tau\right)^{\prime} d \tau
$$

respectivamente, para um SLSM a tempo contínuo dado por,

$$
\dot{x}(t)=A_{\theta(t)} x(t)+B_{\theta(t)} \zeta(t), \quad x(0)=x_{0}, \quad \theta(0) \sim \pi
$$

denotando o processo $\left\{\Phi\left(t_{0}, t\right)\right\}_{t \geq 0}, t_{0} \geq 0$, a matriz de transição de estados da trajetória contínua $x(t)$.

Neste trabalho, definimos a alcançabilidade média (AM) e apresentamos alguns usos deste conceito em um cenário livre de controle; existe uma clara dissimilaridade na concepção da AM quando comparada com noções disponíveis de alcançabilidade estocástica (AE), como veremos no Capítulo 4. Definimos igualmente a controlabilidade média $(\mathrm{CM})$ e usamos este conceito em um cenário em há um controle atuando na dinâmica do sistema em (1.3). Ambas noções de AM e CM para este sistema requerem que o valor esperado dos gramianos respectivos sejam positivos: $\mathcal{E}\left\{\Upsilon_{\text {rch }}(0, t)\right\}>0$ e $\mathcal{E}\left\{\Upsilon_{\text {cnt }}(0, t)\right\}>0$, para algum $t>0, i \in \mathcal{S}$. Contribuímos com a teoria de SLSM ao desenvolver testes computacionais simples para as noções de AM e CM.

Em contraste com o fato de que AM e CM sejam definidas numa maneira simples em termos dos gramianos associados, as tarefas para obter um teste eficiente e estudar o uso destes conceitos em diferentes contextos são bastante mais complexas. De fato, a verificação da AM e CM diretamente pela definição é obviamente inviável. Para superar isto, fomos à procura de condições testáveis para AM e CM estudando equações em certas variáveis matriciais

noção estudada. A palavra fraca tem diferentes usos na matemática aplicada, tendo pouca relação com este trabalho; além disso, no caso da AM em particular, não há necessariamente enfraquecimento de noções prévias de alcançabilidade, veja Exemplo 3. 
$S_{i}$ e $\mathcal{C}_{i}$ respectivamente, mostrando a igualdade dos espaços nulos de $S_{i}$ com $\mathcal{E}\left\{\Upsilon_{\mathrm{rch}}(0, t) \mid \theta(0)=i\right\}$ e de $\mathcal{C}_{i} \operatorname{com} \mathcal{E}\left\{\Upsilon_{\text {cnt }}(0, t) \mid \theta(0)=i\right\}$. Isto envolve as derivadas de $S_{i}$ e $\mathcal{C}_{i}$ em relação ao tempo, avaliadas pela reformulação de propriedades de invariância em (Narváez e Costa, 2011), que pela sua vez requer usar um sistema auxiliar envolvendo as matrizes $A^{\prime}, B^{\prime}$ e uma cadeia "reversa no tempo" para explorar a relação dual com observabilidade média. As derivadas de $S_{i}$ e $\mathcal{C}_{i}$ em relação ao tempo permitem a construção de coleções de matrizes cujo posto é importante para o teste da AM e da CM, como apresentado nos Teoremas 1 e 5 respectivamente.

Quanto aos usos das noções de AM, abordamos primeiramente a positividade das matrizes $X_{i}=\mathcal{E}\left\{x(t) x(t) 1_{\{\theta=i\}}\right\}$, com a dinâmica de $x(t)$ na equação em (1.3) sendo perturbada aditivamente por um processo de Wiener $\{w(t)\}_{t \geq 0}$ no lugar do processo $\{\zeta(t)\}_{t \geq 0}$. O segundo uso das noções de AM que consideramos aqui é no cenário de filtragem, com aplicações em problemas reais. De fato, os resultados neste tópico decorrem mais diretamente da positividade das matrizes $X_{i}$ (o qual, por sua vez, decorre da AM). Concretamente, mostramos que a AM é condição suficiente para que a esperança da matriz de covariância do erro de estimação de estimadores lineares em geral e, em particular de estimadores lineares markovianos, é definida positiva com a interpretação de que suas estimativas contêm um "nível mínimo de aleatoriedade". Vale a pena dizer que o estimador ótimo desta classe de estimadores é o estimador linear quadrático médio mínimo (ELQMM) proposto para SLSM por Fragoso e Costa (2010). Apesar da presença de ruído prejudicar as estimativas dadas pelo filtro, é precisamente ela que nos dá os argumentos para estudar o que chamamos de estabilidade do estimador, uma característica relevante para filtros (Costa e Astolfi, 2009; Nishimura, 1967; Sangsuk-Iam e Bullock, 1990). Estabilidade fundamentalmente significa a invariância (às perturbações na matriz de covariância do erro inicial) da seguinte propriedade: limitação superior para a esperança da matriz de covariância do erro. Para filtros instáveis, a covariância do erro poderia tender para infinito, deteriorando-se rapidamente a qualidade da estimativa. Mostramos que os estimadores lineares markovianos para sistemas AM são estáveis.

Finalmente, quanto às aplicações da CM, consideramos um cenário em que o sistema em (1.3) está sendo perturbado por uma entrada de controle $\{u(t)\}_{t \geq 0}$ no lugar do processo $\{\zeta(t)\}_{t \geq 0}$. Estudamos o que, aqui, chamamos de Factibilidade no problema de controlabilidade, definido pela existência de uma entrada de controle que leve a trajetória contínua $x(t)$ à origem, com 
probabilidade positiva em tempo finito, dada uma condição inicial. Mostramos que CM é uma condição necessária e suficiente para a factibilidade no problema de controlabilidade. 


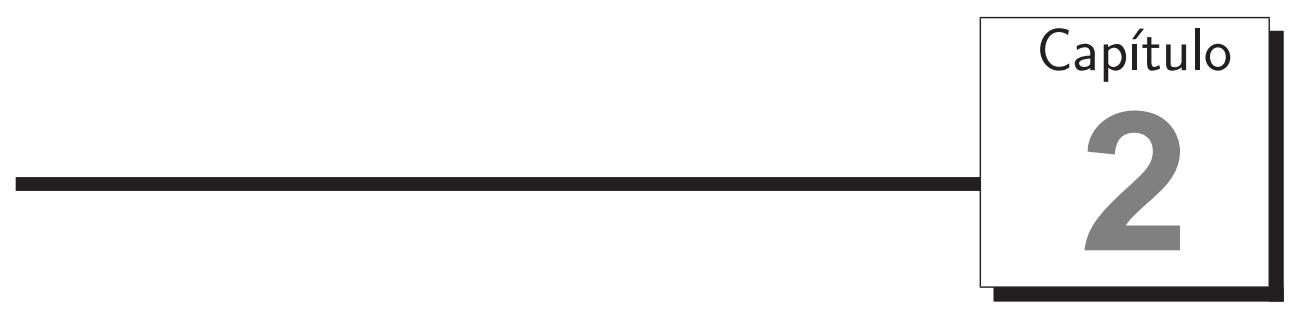

\section{Notações e definições}

Na sequência apresentamos conceitos básicos e alguns resultados bem conhecidos para sistemas lineares, por facilidade de referência. O leitor familiar com estes sistemas pode optar por dirigir-se à Seção 2.2 .

\subsection{Sistemas lineares}

Considere o sistema descrito pelas equações da forma

$$
\begin{aligned}
& \dot{x}(t)=A(t) x(t)+B(t) u(t) \\
& y(t)=C(t) x(t)+D(t) u(t),
\end{aligned}
$$

sendo $x(t) \in \mathbb{R}^{n}, y(t) \in \mathbb{R}^{p}, u(t) \in \mathbb{R}^{m}$ e $A(t), B(t), C(t)$ e $D(t)$ matrizes de dimensões apropriadas. A variável $t$ denota o tempo, $u$ denota a entrada e $y$ a saída do sistema. A primeira equação em (2.1) é chamada de equação de estado e a segunda é chamada equação de saída e ambas equações constituem a descrição espaço-estado de sistemas lineares a tempo contínuo de dimensão finita. Para cada condição inicial $x\left(t_{0}\right)=x_{0}$ e cada entrada dada $u$, a equação de estado possui solução única e contínua para todo $t \in \mathbb{R}$. A solução é dada por:

$$
x(t)=\Phi\left(t, t_{0}\right) x_{0}+\int_{t_{0}}^{t} \Phi(t, \tau) B(\tau) u(\tau) d \tau
$$


com $\Phi\left(t, t_{0}\right)$ denotando a matriz de transição correspondente a $A(t)$. Tal matriz satisfaz a seguintes propriedades (vide (Davis, 1977)):

(a) $\frac{d \Phi(t, \tau)}{d t}=A(t) \Phi(t, \tau) ; \quad \Phi(t, t)=I$

(b) $\Phi(t, s) \Phi(s, \tau)=\Phi(t, \tau)$

(c) $\operatorname{posto}(\Phi(t, \tau))=n, \forall t, \tau$

(d) $\frac{d \Phi(t, \tau)}{d \tau}=-\Phi(t, \tau) A(t)$

Quando $A(t) \equiv A, B(t) \equiv B, C(t) \equiv C$ e $D(t) \equiv D$, são matrizes constantes (ou seja, o sistema é invariante no tempo) temos

$$
\Phi\left(t, t_{0}\right)=e^{A\left(t-t_{0}\right)}=\sum_{k=0}^{\infty} \frac{\left(t-t_{0}\right)^{k}}{k !} A^{k} .
$$

Em algumas circunstancias surge o interesse em usar a entrada do sistema para transferir o estado de $x_{0}$ até algum outro valor $x_{1}$ em um tempo finito $t_{1}>t_{0}$. Se de fato tal transferência de estado for possível, existe $u(t), t \in$ $\left[t_{0}, t_{1}\right]$, tal que a solução da equação de estado em (2.1) toma a forma:

$$
x_{1}=\Phi\left(t_{1}, t_{0}\right) x_{0}+\int_{t_{0}}^{t_{1}} \Phi\left(t_{1}, \tau\right) B(\tau) u(\tau) d \tau
$$

Se em $(2.3), \hat{x}_{1}=x_{1}-\Phi\left(t_{1}, t_{0}\right) x_{0}, u(t)$ não só leva o estado de $x_{0}$ em $t_{0}$ até $x_{1}$ em $t_{1}$ mas também faz o estado alcançar $\hat{x}_{1}$ em $t_{1}$ começando a partir da origem em $t_{0}$.

Definição 1 (Alcançabilidade). O sistema (2.1) é alcançável em $t_{1}$, ou equivalentemente o par $(A(t), B(t))$ é alcançável em $t_{1}$, se para cada estado $x_{1}$ existe uma entrada $u(t), t \in\left[t_{0}, t_{1}\right]$ para algum tempo finito $t_{0}<t_{1}$, que transfere $o$ estado $x(t)$ da origem em $t_{0}$, até $x_{1}$ em $t_{1}$.

O conceito de alcançabilidade é também conhecido como controlabilidade desde a origem. Agora, se em (2.3), $\hat{x}_{0}=x_{0}-\Phi\left(t_{0}, t_{1}\right) x_{1}$, observamos que $u(t)$ é também capaz de transferir o estado $\hat{x}_{0}$ em $t_{0}$ para a origem em $t_{1}$. Temos portanto o conceito de controlabilidade para a origem ou simplesmente controlabilidade como é comumente conhecido.

Definição 2 (Controlabilidade). O sistema (2.1) é controlável em $t_{0}$, ou equivalentemente o par $(A(t), B(t))$ é controlável em $t_{0}$, se para cada estado $x_{0}$ 
existe uma entrada $u(t), t \in\left[t_{0}, t_{1}\right]$ para algum tempo finito $t_{1}>t_{0}$, que transfere o estado $x(t)$ de $x_{0}$ em $t_{0}$ até a origem em $t_{1}$.

Na continuação definimos matrizes importantes envolvidas nos conceitos de alcançabilidade e controlabilidade. Notaremos em geral, para uma matriz $A$, a sua matriz transposta como $A^{\prime}$.

Definição 3. Definimos:

(i) o gramiano de alcançabilidade do sistema (2.1) como sendo a matriz $n \times n$

$$
\Upsilon_{A}\left(t_{0}, t_{1}\right)=\int_{t_{0}}^{t_{1}} \Phi\left(t_{1}, \tau\right) B(\tau) B(\tau)^{\prime} \Phi\left(t_{1}, \tau\right)^{\prime} d \tau
$$

(ii) o gramiano de controlabilidade do sistema (2.1) como sendo a matriz $n \times n$

$$
\Upsilon_{C}\left(t_{0}, t_{1}\right)=\int_{t_{0}}^{t_{1}} \Phi\left(t_{0}, \tau\right) B(\tau) B(\tau)^{\prime} \Phi\left(t_{0}, \tau\right)^{\prime} d \tau
$$

Um resultado que caracteriza os conceitos de alcançabilidade e controlabilidade é o seguinte.

Proposição 1. O sistema (2.1) é alcançável em $t_{1}$ se e somente se existe $t_{0}<t_{1}$ tal que posto $\left(\Upsilon_{A}\left(t_{0}, t_{1}\right)\right)=n$. É controlável em $t_{0}$ se e somente se existe $t_{1}>t_{0}$ tal que posto $\left(\Upsilon_{C}\left(t_{0}, t_{1}\right)\right)=n$.

Observação 1. Existe uma relação importante entre alcançabilidade e controlabilidade de sistemas lineares a tempo continuo. Em virtude de $(i)$ e (ii) da Definição 3 temos $\Upsilon_{A}\left(t_{0}, t_{1}\right)=\Phi\left(t_{1}, t_{0}\right) \Upsilon_{C}\left(t_{0}, t_{1}\right) \Phi\left(t_{1}, t_{0}\right)^{\prime}$ e portanto posto $\left(\Upsilon_{A}\left(t_{0}, t_{1}\right)\right)=\operatorname{posto}\left(\Upsilon_{C}\left(t_{0}, t_{1}\right)\right)$ já que $\Phi\left(t_{1}, t_{0}\right)$ é não singular para todo $t_{0}$ e $t_{1}$. Este é o fato chave na relação entre alcançabilidade e controlabilidade para sistemas lineares variantes no tempo: são conceitos equivalentes. Não ocorre tal propriedade no caso a tempo discreto (controlabilidade não necessariamente implica em alcançabilidade) pois em geral a matriz de transição $\Phi$ não é inversível.

Em sistemas lineares contínuos invariantes no tempo a diferença $T=t_{1}-$ $t_{0}>0$, ao invés dos tempos individuais $t_{0}$ e $t_{1}$, têm um papel importante. Neste caso, consideramos $t_{0}=0$ e $t_{1}=T$ e as definições e propriedades de controlabilidade e alcançabilidade são adaptadas a estas considerações. Seja a matriz de controlabilidade $n \times m n$ (ou de alcançabilidade mais precisamente) definida como

$$
\mathbb{C}(A, B)=\left[\begin{array}{llll}
B & A B & \cdots & A^{n-1} B
\end{array}\right]
$$


Proposição 2 (Teste do posto da matriz de controlabilidade ${ }^{a}$ ). Um sistema linear contínuo invariante no tempo é controlável se, e somente se,

$$
\text { posto } \mathbb{C}(A, B)=n \text {. }
$$

O teste anterior vem do fato de que o conjunto imagem de $\Upsilon_{A}(0, T)$ é igual ao conjunto imagem da matriz $\mathbb{C}(A, B)$ (vide por exemplo Antsaklis e Michel (2006)), independente do valor $T>0$. Além disso, $\mathbb{C}(A, B)$ é invariante respeito da matriz $A$, isto é, para qualquer $x$ no conjunto imagem de $\mathbb{C}(A, B)$ temos que $A x$ também pertence a este conjunto. Por outro lado, consideramos também o cenário em que há perturbações aditivas na equação de estado do sistema em (2.1). Neste sentido, a equação de estado em (2.1) torna-se:

$$
d x(t)=A x(t) d t+B u(t) F d w(t), \quad x(0)=x_{0}
$$

nos limitando apenas ao caso invariante no tempo; $F$ é uma matriz de dimensão $n \times q$ e $\{w(t)\}_{t \geq 0}$ é um processo de Wiener $q$-dimensional. Definições e caracterizações de controlabilidade existentes na literatura para este tipo de sistemas são apresentadas a seguir.

Definição 4 (Zabczyk (1981)). Dizemos que o sistema em (2.7) é: i) Fracamente controlável se para todo $x_{0} \in \mathbb{R}^{n}$, e qualquer conjunto aberto não vazio $V \subset \mathbb{R}^{n}$, existe um controle $\{u(t)\}_{t \geq 0}$ tal que $x\left(T ; x_{0}\right) \in V$ com probabilidade positiva, para algum $T>0$; ii) Controlável se $x\left(T ; x_{0}\right) \in V$ com probabilidade igual a 1; iii) Fortemente controlável se o tempo de alcance $T=i n f_{t>0}\left\{x\left(t ; x_{0}\right) \in V\right\}$ tem valor esperado finito.

O segundo argumento em $x\left(T ; x_{0}\right)$ indica o fato de que o processo em consideração inicia em $x_{0}$.

Proposição 3 (Zabczyk (1981)). O sistema em (2.7) é fracamente controlável se e somente se

$$
\text { posto }\left[\begin{array}{llllllll}
B & A B & \cdots & A^{n-1} B & F & A F & \cdots & A^{n-1} F
\end{array}\right]=n .
$$

Quando $B=0$, a equação em (2.7) é denominada equação diferencial estocástica. Neste caso dizemos simplesmente que o sistema $(A, F)$ é controlável

a Para sistemas lineares contínuos invariantes no tempo falamos só em controlabilidade e desconsideramos a alcançabilidade pois os dois conceitos são equivalentes. 
(vide Zakai e Snyders (1970)) se e somente se posto $\mathbb{C}(A, F)=n$. Se assumirmos $d w(t)$ como um "controle", temos que a sua escolha é dada de maneira aleatória e irregular. Assim, se o par $(A, F)$ é controlável segue que $\{x(t)\}_{t \geq 0}$ deveria ir em todo lugar no sentido exposto no item $i$ ) da Definição 4 . Na sequência apresentamos uma caracterização da controlabilidade de $(A, F)$ relacionada com o segundo momento da trajetória $x(t)$.

Proposição 4 (Davis (1977)). A matriz de covariância do processo $\{x(t)\}_{t \geq 0}$ é não singular para todo $t \geq 0$ se e somente se o par $(A, F)$ é fracamente controlável.

Outra propriedade desejável em sistemas lineares é a capacidade de determinar o estado a partir da observação da saída em tempo finito já que frequentemente é difícil ou impossível medir o estado do sistema diretamente. Tais propriedades levam aos conceitos de observabilidade e construtibilidade. Estes conceitos, suas caracterizações e as relações de dualidade que eles têm com a alcançabilidade e a controlabilidade podem ser vistos no Apêndice A.

\subsection{Sistemas lineares com saltos markovianos}

Seja $\mathbb{Z}^{0}$ o conjunto dos inteiros não negativos. Seja $\mathfrak{R}^{n, q}$ (respectivamente $\mathfrak{R}^{n}$ ) o espaço vetorial formado pelas matrizes de tamanho $n \times q$ (respectivamente $n \times n)$ e $\mathfrak{R}^{r 0}$ o cone convexo de matrizes semi-definidas positivas $\left\{U \in \mathfrak{R}^{r}: U=U^{\prime} \geq 0\right\}\left(\mathfrak{R}^{r+}\right.$ quando as matrizes são definidas positivas). Para $U \in \mathfrak{R}^{n, q}, \mathcal{N}(U)$ e $\mathcal{I} m(U)$ denotam o espaço nulo e o espaço imagem de $U$ respectivamente. $U \geq V$ significa que $U-V \in \mathfrak{R}^{r 0}$ e $U>V$ significa $U-V \in \mathfrak{R}^{r+}$. Notamos a função indicadora por $1_{\{.\}}$, a função esperança matemática é notada por $\mathcal{E}\{\cdot\}$ e a traça de uma matriz por $\operatorname{tr}\{$.$\} . Seja \mathcal{M}^{r, n}$ o espaço vetorial formado por tuplas de $N$ matrizes definido como $\mathcal{M}^{r, n}=\left\{U=\left(U_{1}, \ldots, U_{N}\right): U_{j} \in \mathfrak{R}^{r, n}, j=1, \ldots, N\right\}$; também, $\mathcal{M}^{r} \equiv \mathcal{M}^{r, r}$. Denotamos por $\mathcal{M}^{r 0}\left(\mathcal{M}^{r+}\right)$ o conjunto $\mathcal{M}^{r}$ quando este é formado por $U_{j} \in \mathfrak{R}^{r 0}\left(U_{j} \in \mathfrak{R}^{r+}\right)$ para todo $j=1, \ldots, N$. $\mathcal{M}^{r, n}$ com o produto interno dado por

$$
\langle U, V\rangle=\sum_{j=1}^{N} \operatorname{tr}\left\{U_{j}^{\prime} V_{j}\right\}
$$

é um espaço de Hilbert. Usamos a norma $\|U\|=\langle U, I\rangle$ em $\mathcal{M}^{n 0}$. 
Formalmente, vamos considerar o sistema ${ }^{\mathrm{b}}$ definido sobre o espaço de probabilidade $\left(\Omega, \mathfrak{F},\left\{\mathfrak{F}_{t}\right\}, \mathcal{P}\right)$ por:

$$
\begin{aligned}
& \dot{x}(t)=A_{\theta(t)} x(t)+B_{\theta(t)} \zeta(t), \quad x(0)=x_{0}, \quad \theta(0) \sim \pi \\
& y(t)=C_{\theta(t)} x(t)+D_{\theta(t)} \eta(t)
\end{aligned}
$$

sendo $x(t) \in \mathbb{R}^{n}, y(t) \in \mathbb{R}^{p}$ e $x_{0}$ uma variável aleatória satisfazendo $\mathcal{E}\left\{x_{0}\right\}=\bar{x}_{0}$ e $\mathcal{E}\left\{x_{0} x_{0}^{\prime}\right\}=\Psi$. Os processos de entrada $\{\zeta(t)\}_{t \geq 0}$ e $\{\eta(t)\}_{t \geq 0}$ perturbam aditivamente a dinâmica do sistema e a saída $y(t)$ respectivamente. Como antes dito, $\{\theta(t)\}_{t \geq 0}$ é uma cadeia de Markov geral com espaço de estados finito, isto é, $\theta(t) \in \mathcal{S}=\{1, \ldots, N\}$ para todo $t \geq 0$; a matriz $N \times N$ das taxas de probabilidades de transição associada à cadeia é dada por:

$$
\Lambda=\left[\lambda_{i j}\right] \text {, tal que } \lambda_{i j} \geq 0 \text { para } i \neq j \text {, e } \sum_{j=1}^{N} \lambda_{i j}=0 \text {. }
$$

Em cada instante $t$ temos que $A_{\theta(t)}=A_{i}$ sempre que $\theta(t)=i$, sendo $A_{i}$ tomada de uma coleção de matrizes $A=\left(A_{1}, \ldots, A_{N}\right)$ e similarmente para as matrizes coeficientes $B_{i}, i \in \mathcal{S}$; em certas ocasiões, notaremos o sistema (2.8) simplesmente pela tupla $(A, B, C, D, \Lambda)$. Consideramos $\mathbb{P}(t)=\left[p_{i j}(t)\right], t \geq 0$, como sendo o semi-grupo de transição sobre $\mathcal{S}$, com $p_{i j}(t)=\mathcal{P}(\theta(t+s)=$ $j \mid \theta(s)=i), i, j \in \mathcal{S}$. Fazemos $p(t)=\left[p_{1}(t), \ldots, p_{N}(t)\right]$ sendo $p_{i}(t)=\mathcal{P}(\theta(t)=$ i) e $p(0)=\pi$ a distribuição inicial da cadeia de Markov. As taxas de transição são tais que $\lambda_{i j}=\lim _{\Delta t \rightarrow 0}\left[\mathcal{P}(\theta(t+\Delta t)=j \mid \theta(t)=i)-1_{\{i=j\}}\right] / \Delta t$. Para $\pi$ dada, definimos o conjunto:

$$
\mathcal{S}_{\pi}=\{j \in \mathcal{S}: \exists s \geq 0 \text { tal que } \mathcal{P}(\theta(s)=j)>0\}
$$

o qual contém os estados de Markov com distribuição de probabilidade inicial positiva junto com estados que podem ser alcançados a partir destes. $\mathcal{S}_{\pi \text {,rec }}$ representa o subconjunto de $\mathcal{S}_{\pi}$ que contém os estados de Markov recorrentes. Para um estudo mais detalhado sobre cadeias de Markov veja por exemplo Bhattacharya e Waymire (1990); Norris (1998).

Na literatura de SLSM existem algumas definições e propriedades correspondentes ao conceito de controlabilidade e que são usadas em diversos pro-

\footnotetext{
${ }^{\mathrm{b}} \mathrm{A}$ definição do sistema (2.8) poderá sofrer algumas variações quanto às variáveis $\zeta(t) \mathrm{e}$ $\eta(t)$; por exemplo, no Capítulo 5 serão considerados processos de Wiener no lugar destes processos.
} 
blemas. Tais definições e resultados são obtidos para um SLSM dado pela equação de estado em (2.8), num cenário em que há presença de uma entrada de controle $u(t)$ no lugar da variável $\zeta(t)$; ou seja, se considera um SLSM dado por:

$$
\dot{x}(t)=A_{\theta(t)} x(t)+B_{\theta(t)} u(t) .
$$

Definição 5 (Mariton (1987)). Dizemos que um SLSM é: i) Fracamente controlável se para todo $x_{0} \in \mathbb{R}^{n}, x_{1} \in \mathbb{R}^{n}$, e todo $\epsilon>0$, existe um tempo aleatório $T$ (quase certamente) finito e uma lei de controle u definida sobre $[0, T]$ tal que $\mathcal{P}\left(\left\|x\left(T ; x_{0}\right)-x_{1}\right\| \leq \epsilon\right)>0$; ii) Controlável se $\mathcal{P}\left(\left\|x\left(T ; x_{0}\right)-x_{1}\right\| \leq \epsilon\right)=1$; iii) Fortemente controlável se o tempo de alcance $T=i n f_{t>0}\left\{\left\|x\left(t ; x_{0}\right)-x_{1}\right\| \leq \epsilon\right\}$ tem esperança finita, isto é, $\mathcal{E}\{T\}<\infty$.

Apresentamos também a seguinte versão de controlabilidade estocástica.

Definição 6 (Ji e Chizeck (1990)). Dizemos que um SLSM é estocasticamente controlável se, para qualquer $x_{0} \in \mathbb{R}^{n}, i_{0} \in \mathcal{S}$ e dado um $T>0$ finito, existe uma lei de controle de realimentação de estado $u(t)=L_{\theta(t)} x(t)$ para $0 \leq t \leq T$ tal que $\mathcal{E}\left\{x\left(T ; x_{0}\right)^{\prime} x\left(T ; x_{0}\right)\right\}<\epsilon$.

Caracterizações, propriedades e/ou aplicações das definições anteriores podem ser vistas nas referências citadas respectivas. Relacionado à noção de alcançabilidade, existe também na literatura o chamado problema de alcançabilidade estocástica para sistemas estocásticos híbridos em geral. Tal problema consiste em determinar a probabilidade de certos conjuntos de alcançabilidade em um tempo finito pre-especificado, como exposto em (Bujorianu e Lygeros, 2007). Neste trabalho propomos novas definições de alcançabilidade e controlabilidade para SLSM diferentes às aqui mencionadas como veremos posteriormente. Para tal fim precisaremos de mais algumas ferramentas que serão definidas na sequência.

Seguindo Costa et al. (2005b), usaremos os operadores lineares e inversíveis vec e $\varphi$, com o operador vec empilhando as colunas de uma matriz em um vetor coluna simples, e $\varphi$ sendo definido de uma forma tal que, para $U \in \mathcal{M}^{n}$, $\varphi(U)=\left[\operatorname{vec}\left(U_{1}\right)^{\prime} \operatorname{vec}\left(U_{2}\right)^{\prime} \cdots \operatorname{vec}\left(U_{N}\right)^{\prime}\right]^{\prime}$. 
Sejam também os operadores $\mathcal{L}_{A}, \mathcal{T}_{A}, \mathcal{H}_{A}: \mathcal{M}^{n} \longrightarrow \mathcal{M}^{n}$, definidos como:

$$
\begin{gathered}
\mathcal{L}_{A, i}(U)=A_{i}^{\prime} U_{i}+U_{i} A_{i}+\sum_{j=1}^{N} \lambda_{i j} U_{j}, \\
\mathcal{T}_{A, i}(U)=A_{i} U_{i}+U_{i} A_{i}^{\prime}+\sum_{j=1}^{N} \lambda_{j i} U_{j}, \\
\mathcal{H}_{A, i}(U)=-A_{i} U_{i}-U_{i} A_{i}^{\prime}+\sum_{j=1}^{N} \lambda_{i j} U_{j}
\end{gathered}
$$

para $j=1, \ldots, N$. Denotamos $\mathcal{L}_{A}^{0}(U)=U$ e $\mathcal{L}_{A}^{k}(U)=\mathcal{L}_{A}\left(\mathcal{L}_{A}^{k-1}(U)\right)$, para $k \geq 1$; usamos uma notação semelhante para $\mathcal{T}_{A}$ e $\mathcal{H}_{A}$. Os operadores $\mathcal{T}_{A}$ e $\mathcal{L}_{A}$ são operadores adjuntos entre si e $\mathcal{L}_{-A^{\prime}}=\mathcal{H}_{A}$. A notação empregada apenas enfatiza $A$ como parâmetro do operador pois o outro parâmetro envolvido, $\Lambda$, é sempre fixo; o sinal negativo em $\mathcal{H}$ no parâmetro $A$ será explicado oportunamente. Para $U, V \in \mathcal{M}^{n}$, é simples verificar que $\langle U, V\rangle=\varphi(U)^{\prime} \varphi(V)$. Como em (Costa e do Val, 2002a), usando esta notação podemos escrever $\varphi\left(\mathcal{L}_{A}(U)\right)=\mathcal{A} \varphi(U)$, e $\varphi\left(\mathcal{T}_{A}(U)\right)=\mathcal{A}^{\prime} \varphi(U)$, sendo

$$
\mathcal{A}=\left[\begin{array}{ccc}
\hat{A}_{1}+\lambda_{11} \hat{I} & \ldots & \lambda_{1 N} \hat{I} \\
\lambda_{21} \hat{I} & \ddots & \lambda_{2 N} \hat{I} \\
\vdots & \vdots & \vdots \\
\lambda_{N 1} \hat{I} & \ldots & \hat{A}_{N}+\lambda_{N N} \hat{I}
\end{array}\right]
$$

com $\hat{I}=I \in \mathfrak{R}^{n^{2}}, \hat{A}_{j}=V \otimes A_{j}^{\prime}+A_{j}^{\prime} \otimes V$, e $V=I \in \mathfrak{R}^{n}$, sendo que $\otimes$ denota o produto de Kronecker. 


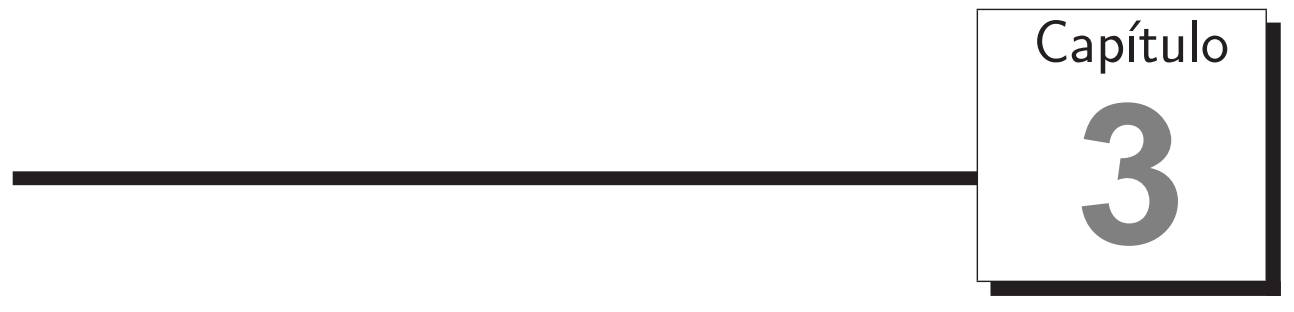

\section{Resultados preliminares}

Neste capítulo exploramos uma conexão entre os gramianos de alcançabilidade/controlabilidade e o de observabilidade.

\subsection{Propriedades do gramiano de observabilidade de SLSM}

Seja o sistema auxiliar $(\tilde{A}, \tilde{B}=0, \tilde{C}, \tilde{D}=0, \tilde{\Lambda})$,

$$
\begin{aligned}
\dot{x}(t) & =\tilde{A}_{\tilde{\theta}(t)} x(t), \quad x(0)=x_{0}, \quad \tilde{\theta}(0) \sim \tilde{\pi}, \\
y(t) & =\tilde{C}_{\tilde{\theta}(t)} x(t),
\end{aligned}
$$

e o gramiano de observabilidade associado

$$
\Upsilon_{\text {obs }}(0, t)=\int_{0}^{t} \tilde{\Phi}(\tau, 0)^{\prime} \tilde{C}_{\tilde{\theta}(\tau)}^{\prime} \tilde{C}_{\tilde{\theta}(\tau)} \tilde{\Phi}(\tau, 0) d \tau
$$

sendo $\tilde{\Phi}(\tau, 0)$ a matriz de transição de estado envolvida na solução de (3.1). Considere

$$
W^{t}(x, j)=x^{\prime} \mathcal{E}\left\{\Upsilon_{\text {obs }}(0, t) \mid \tilde{\theta}(0)=j\right\} x,
$$

para todo $x \in \mathbb{R}^{n}$ e $j \in \mathcal{S}$, o qual é usualmente interpretado como um funcional de custo. Vamos considerar também a sequência de coleções de matrizes $O(k) \in$ 
$\mathcal{M}^{n}$, definidas recursivamente conforme (Costa e do Val, 2002a) por

$$
\begin{aligned}
O_{j}(k+1) & =\mathcal{L}_{\tilde{A}, j}(O(k)), \quad k \in \mathbb{Z}^{0}, j \in \mathcal{S} \\
O_{j}(0) & =\tilde{C}_{j}^{\prime} \tilde{C}_{j} .
\end{aligned}
$$

Seja também $L(t), t \geq 0$, definido pela equação linear diferencial:

$$
\dot{L}_{j}(t)=\mathcal{L}_{\tilde{A}, j}(L(t))+\tilde{C}_{j}^{\prime} \tilde{C}_{j}, \quad L(0)=0, \quad t \geq 0
$$

para cada $j \in \mathcal{S}$. Note que para cada $j \in \mathcal{S}$ se cumpre:

$$
O_{j}(k)=\frac{d^{k+1} L_{j}}{d t^{k+1}}(0)
$$

O seguinte encontra-se em (Costa e do Val, 2002a) e apresenta a relação entre a função $L$ e o funcional $W^{t}(\cdot, j)$.

Proposição 5. A seguinte afirmação é válida:

$$
W^{t}(x, j)=\int_{0}^{t}\left\langle U(\tau), \tilde{C}^{\prime} \tilde{C}\right\rangle d \tau=\langle U, L(t)\rangle,
$$

sendo $\dot{U}_{j}(t)=\mathcal{T}_{\tilde{A}, j}(U(t))$, para todo $j \in \mathcal{S}$, com $U_{j}=x x^{\prime}$ para $\tilde{\theta}(0)=j$ e $U_{i}=0, i \neq j$. Em geral temos que:

$$
W^{t}(U)=\int_{0}^{t}\left\langle U(\tau), \tilde{C}^{\prime} \tilde{C}\right\rangle d \tau=\langle U, L(t)\rangle, \quad \text { para } \quad U \in \mathcal{M}^{n}
$$

As matrizes $O_{j}$ cumprem um papel importante na caracterização da observabilidade de $(\tilde{A}, 0, \tilde{C}, 0, \tilde{\Lambda})$ conforme a seguinte definição (veja (Costa e do Val, 2002a)).

Definição 7 (Observabilidade média). As matrizes $\mathcal{O}_{j} \in \mathcal{M}^{n\left(n^{2} N\right), n}, j \in \mathcal{S}_{\pi}$, dadas como:

$$
\mathcal{O}_{j}=\left[\begin{array}{llll}
O_{j}^{\prime}(0) & O_{j}^{\prime}(1) & \ldots & O_{j}^{\prime}\left(n^{2} N-1\right)
\end{array}\right]^{\prime}
$$

são denominadas matrizes de observabilidade do sistema $(\tilde{A}, 0, \tilde{C}, 0, \tilde{\Lambda})$. Se $\mathcal{O}_{j}$ é de posto completo para cada $j \in \mathcal{S}_{\pi}$ então o sistema é $O M$.

O resultado a seguir apresenta uma aproximação da invariância do espaço nulo de $L$ em relação às suas derivadas avaliadas em zero as quais constituem as matrizes de observabilidade, sendo portanto esta uma propriedade associada à $\mathrm{OM}$ do sistema como explicado em (Costa e do Val, 2002a). 
Lema 1. a Sejam $x \in \mathbb{R}^{n}, j \in \mathcal{S}_{\pi}, V \in \mathcal{M}^{n}$ definida como $V_{j}=x x^{\prime}$ e $V_{i}=0$, $\forall i \neq j$ e $\ell(t), w \in \mathbb{R}^{n^{2} N}$ definidos como $\ell(t)=\varphi(L(t))$ e $w=\varphi(V)$. As seguintes afirmações são equivalentes:

(i) $w^{\prime} \frac{d^{k+1} \ell}{d t^{k+1}}(0)=0$, para $k=0, \ldots, n^{2} N-1$.

(ii) $x \in \mathcal{N}\left(L_{j}(t)\right)$ ou, equivalentemente, $w^{\prime} \ell(t)=0$ para todo $t \geq 0$.

Da Proposição 5 e do Lema 1 deriva-se o resultado a seguir, o qual foi desenvolvido em (Narváez e Costa, 2010) inspirado em resultados de (Costa e do Val, 2002a).

Corolário 1. $x^{\prime} O_{j}(k) x=0$ para todo $k=0, \ldots, n^{2} N-1$, se e somente se $W^{t}(x, j)=0, t \geq 0$.

Demonstração. Seja $V \in \mathcal{M}^{n}$ definido como $V_{j}=x x^{\prime}$ e $V_{i}=0, \forall i \neq j$ e $w \in \mathbb{R}^{n^{2} N}$ como $w=\varphi(V)$. As seguintes implicações são obtidas de uma maneira simples para cada $k=0, \ldots, n^{2} N-1$,

$$
\begin{aligned}
x^{\prime} O_{j}(k) x=0 \Leftrightarrow x^{\prime} \frac{d^{k+1} L_{j}}{d t^{k+1}}(0) x=0 & \Leftrightarrow\left\langle V, \frac{d^{k+1} L}{d t^{k+1}}(0)\right\rangle=0 \\
& \Leftrightarrow w^{\prime} \frac{d^{k+1} \ell}{d t^{k+1}}(0)=0,
\end{aligned}
$$

sendo $\ell(t)=\varphi(L(t)) \in \mathbb{R}^{n^{2} N}$ para todo $t \geq 0$. De (3.10) e o Lema 1 temos que $x^{\prime} O_{j}(k) x=0, k=0, \ldots, n^{2} N-1$, é equivalente a $0=w^{\prime} \ell(t)=\langle V, L(t)\rangle$, $t \geq 0$; $\log$ o (3.8) completa a prova.

No lema anterior vimos a relação existente entre o funcional de custo $W^{t}$ e as matrizes de observabilidade $O_{j}$ que quase caracteriza o conceito de $\mathrm{OM}$ exceto pelo fato de que as matrizes $O_{j}$ não necessariamente são definidas positivas. A respeito, uma consequência indesejável da positividade das matrizes $O_{j}$ seria a possibilidade de que ocorra que $W^{t}(x, j)=\infty$. Porém, estes resultados prévios, partindo da hipótese de que $x^{\prime} O_{j}(k) x=0$, são ferramenta importante para garantir que as derivadas de $x(t)$ permanecem no espaço nulo de $C_{\tilde{\theta}}$ sempre que $\tilde{\theta}$ seja acessível de $j$, levando finalmente na caracterização da OM.

Lema 2. Assuma que a sequência de estados $\tilde{\theta}(t), i_{1}, i_{2}, \ldots, i_{m-1}, i_{m} d a c a$ deia $\tilde{\theta}$ é tal que $\tilde{\lambda}_{\tilde{\theta}(t), i_{1}} \tilde{\lambda}_{i_{1}, i_{2}} \cdots \tilde{\lambda}_{i_{m-1}, i_{m}} \neq 0$. Se $Q \tilde{A}_{\tilde{\theta}(t)}^{p} x(t)=0$ (q.c.), $t \geq 0$, então $Q \tilde{A}_{i_{m}}^{p} x(t)=0$ (q.c.), $t \geq 0, p \geq 0$.

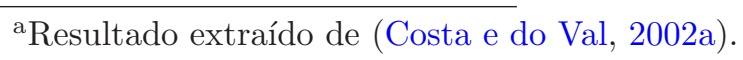


Demonstração. Assumiremos o contrário, isto é, que existe $T$, tal que $Q \tilde{A}_{i_{m}}^{p} x(T) \neq 0$ com probabilidade positiva $\alpha$, ou seja, $P(x(T) \notin \mathcal{N})=\alpha>0$, $\operatorname{com} \mathcal{N}=\mathcal{N}\left(Q \tilde{A}_{i_{m}}^{p}\right)$. Então, usando (Narváez e Costa, 2010, Proposição 2), podemos mostrar que para uma certa vizinhança $\mathcal{V}$ de $x(T)$, existe $\bar{t}>0$ (possivelmente dependendo de $x(T)$ ) tal que $x(l) \in \mathcal{V}, T<l \leq T+\bar{t}$, com $\mathcal{N} \cap \mathcal{V}=\emptyset$. Ou seja:

$$
Q \tilde{A}_{i_{m}}^{p} x(l) \neq 0, \quad \text { (q.c.) } \quad T<l \leq T+\bar{t} .
$$

Agora, assumimos que $T_{1}$ seja o tempo da primeira visita ao estado $i_{m}$ começando de $T$, isto é, $T_{1}=\inf _{t \geq 0}\left\{t \geq T: \tilde{\theta}(t)=i_{m}\right\}-T$, então temos que $P\left(T_{1}<\bar{t}\right)>0$. Assim,

$$
\begin{aligned}
P\left(Q \tilde{A}_{\tilde{\theta}(l)}^{p} x(l) \neq 0\right)= & P\left(Q \tilde{A}_{\tilde{\theta}(l)}^{p} x(l) \neq 0 \mid x(T) \in \mathcal{N}\right)(1-\alpha) \\
& +P\left(Q \tilde{A}_{\tilde{\theta}(l)}^{p} x(l) \neq 0 \mid x(T) \notin \mathcal{N}\right) \alpha \\
\geq & P\left(Q \tilde{A}_{\tilde{\theta}(l)}^{p} x(l) \neq 0 \mid x(T) \notin \mathcal{N}\right) \alpha
\end{aligned}
$$

e, com $l=T_{1}$ obtemos,

$$
\begin{aligned}
& P\left(Q \tilde{A}_{\tilde{\theta}\left(T_{1}\right)}^{p} x\left(T_{1}\right) \neq 0\right) \geq P\left(Q \tilde{A}_{\tilde{\theta}\left(T_{1}\right)}^{p} x\left(T_{1}\right) \neq 0 \mid x(T) \notin \mathcal{N}\right) \alpha \\
& \quad \geq P\left(Q \tilde{A}_{\tilde{\theta}\left(T_{1}\right)}^{p} x\left(T_{1}\right) \neq 0 \mid x(T) \notin \mathcal{N}, T_{1}<\bar{t}\right) \alpha P\left(T_{1}<\bar{t}\right) \\
& \quad=\alpha P\left(T_{1}<\bar{t}\right)>0
\end{aligned}
$$

o qual contradiz a hipótese que $Q \tilde{A}_{\tilde{\theta}(t)}^{p} x(t)=0$ (q.c.), $t \geq 0$.

$\mathrm{Na}$ continuação obtemos a avaliação chave para mostrar a igualdade dos espaços nulos do funcional de custo $W^{t}$ e as matrizes $O_{j}(k)$.

Corolário 2. Para cada $x \in \mathbb{R}^{n}$ e cada sequência de estados de Markov $j, i_{1}, \ldots, i_{m}, i$ tal que $x^{\prime} O_{j}(k) x=0, k=0, \ldots, n^{2} N-1, e \tilde{\lambda}_{j, i_{1}} \tilde{\lambda}_{i_{1}, i_{2}} \cdots \tilde{\lambda}_{i_{m}, i} \neq$ 0 , temos

$$
\tilde{C}_{i} \tilde{A}_{i_{m}}^{p_{1}} \tilde{A}_{i_{m-1}}^{p_{2}} \cdots \tilde{A}_{j}^{p_{m}} x=0
$$

para cada inteiro $p_{\ell} \geq 0, \ell=1, \ldots, m$.

Demonstração. Se para cada $k=0, \ldots, n^{2} N-1$ nós temos $x^{\prime} O_{j}(k) x=0$ então, usando (Narváez e Costa, 2010, Corolários 1 e 2) obtemos

$$
\tilde{C}_{i} x(t)=0 \quad \text { (q.c.), } \quad t \geq 0
$$


e também (vide (Narváez e Costa, 2010, Lema 4))

$$
\tilde{C}_{i} \tilde{A}_{\theta(t)}^{p_{1}} x(t)=0 \quad \text { (q.c.), } \quad \forall t \geq 0 .
$$

Assuma que $\tilde{\theta}(t)=j$ (desde que $\tilde{\theta}(0)=j$ temos que $\tilde{\theta}(t)=j$ com probabilidade positiva). Por hipótese, $\tilde{\theta}(t)$ alcança $i_{m}$ e, assim, aplicando o Lema 2 obtemos,

$$
\tilde{C}_{i} \tilde{A}_{i_{m}}^{p_{1}} x(t)=0 \quad \text { (q.c.), } \quad \forall t \geq 0 .
$$

Se fizermos $Q=\tilde{C}_{i} \tilde{A}_{i_{m}}^{p_{1}}$, procedendo similarmente como antes (substituindo $\tilde{C}_{i}$ por $Q$ em (3.12)), obtemos

$$
Q \tilde{A}_{\tilde{\theta}(t)}^{p_{2}} x(t)=0 \quad \text { (q.c.) } \quad \forall t \geq 0
$$

e, lembrando que assumimos $\tilde{\theta}(t)=i$, temos novamente por hipótese que $\tilde{\theta}(t)$ alcança $i_{m-1}$, levando em

$$
\tilde{C}_{i} \tilde{A}_{i_{m}}^{p_{1}} \tilde{A}_{i_{m-1}}^{p_{2}} x(t)=0 \quad \text { (q.c.) } \quad \forall t \geq 0
$$

Procedendo recursivamente,

$$
\tilde{C}_{i} \tilde{A}_{i_{m}}^{p_{1}} \tilde{A}_{i_{m-1}}^{p_{2}} \cdots \tilde{A}_{j}^{p_{m}} x(t)=0 \quad \text { (q.c.) } \quad \forall t \geq 0
$$

e, em particular para $t=0$, podemos ver que (3.11) se cumpre.

Exemplo 1. Considere $(\tilde{A}, 0, \tilde{C}, 0, \tilde{\Lambda})$ com

$$
\begin{array}{ll}
\tilde{A}_{1}=\left[\begin{array}{ccc}
2 & 0 & 0 \\
0.01 & 1 & 0 \\
0.9 & 1 & 0.1
\end{array}\right], \quad \tilde{C}_{1}=\left[\begin{array}{lll}
1 & 0 & 0 \\
0 & 0 & 0 \\
0 & 0 & 0
\end{array}\right], \\
\tilde{A}_{2}=\left[\begin{array}{ccc}
0.5 & 0 & 0 \\
0 & 0.99 & 0 \\
1 & 1 & 1
\end{array}\right], \quad \tilde{C}_{2}=\left[\begin{array}{lll}
0 & 1 & 0 \\
0 & 0 & 0 \\
0 & 0 & 0
\end{array}\right],
\end{array}
$$




$$
\tilde{A}_{3}=\left[\begin{array}{ccc}
1 & 0 & 0 \\
0.1 & 0.25 & 0 \\
0 & 0 & 1
\end{array}\right], \quad \tilde{C}_{3}=0, \quad \tilde{\Lambda}=\left[\begin{array}{ccc}
-3 & 1 & 2 \\
2 & -5 & 3 \\
0.5 & 0.5 & -1
\end{array}\right] .
$$

Note que $\mathcal{S}=\{1,2,3\}, n=3, N=3$. Consideramos a condição inicial $(x(0), \tilde{\theta}(0)) \operatorname{com} x(0)=\left[\begin{array}{lll}0 & 0 & 1\end{array}\right]^{\prime} e \tilde{\theta}(0)=1$ (compativel com a distribuição inicial $\tilde{\pi}=\left[\begin{array}{lll}1 & 0 & 0\end{array}\right]$ ). Considere a sequência $1,3,1,2,3,2$, para a qual $\tilde{\lambda}_{1,3} \tilde{\lambda}_{3,1} \tilde{\lambda}_{1,2} \tilde{\lambda}_{2,3} \tilde{\lambda}_{3,2} \neq 0$. Verificamos que $x^{\prime} O_{1}(k) x=0, k=0, \ldots, 26$, satisfazendo assim as hipóteses do Corolário $2, e$

$$
\tilde{C}_{2} \tilde{A}_{3}^{p_{1}} \tilde{A}_{2}^{p_{2}} \tilde{A}_{1}^{p_{3}} \tilde{A}_{3}^{p_{4}} \tilde{A}_{1}^{p_{5}} x(0)=0
$$

para cada $p_{\ell}=0, \ldots, 20, \ell=1, \ldots, 5$. Isto confirma (3.11).

O seguinte é uma reunião dos resultados nos Corolários 1 e 2. A propriedade de invariância apresentada para o espaço nulo de $\tilde{C}$ é parecida com um resultado para sistemas com leis de chaveamento geral como em (Sun e Ge, 2005, Teorema 4.26).

Proposição 6. Consideremos o sistema $(\tilde{A}, \tilde{B}=0, \tilde{C}, \tilde{D}=0, \tilde{\Lambda})$. Para cada $x \in \mathbb{R}^{n}$, as seguintes afirmações são equivalentes:

(i) $W^{t}(x, j)=0, t \geq 0, j \in \mathcal{S}_{\pi}$.

(ii) $x^{\prime} O_{j}(k) x=0, k \in \mathbb{Z}^{0}, j \in \mathcal{S}_{\pi}$.

(iii) $\tilde{C}_{i} \tilde{A}_{i_{m}}^{p_{0}} \tilde{A}_{i_{m-1}}^{p_{1}} \cdots \tilde{A}_{j}^{p_{m}} x=0$, para qualquer sequência de estados de Markov $j, i_{1}, \ldots, i_{m}, i$ contida em $\mathcal{S}_{\pi}$ tal que $\tilde{\lambda}_{j, i_{1}} \tilde{\lambda}_{i_{1}, i_{2}} \cdots \tilde{\lambda}_{i_{m}, i} \neq 0$ com $p_{\ell} \geq 0$ $\ell=0, \ldots, m$.

Demonstração. $(i) \Leftrightarrow(i i)$. Veja Corolário $1(i i) \Rightarrow($ iii $)$. Veja Corolário 2.(iii) $\Rightarrow($ ii $)$. Temos

$$
x^{\prime} \tilde{A}_{j}^{\prime q_{k}} \cdots \tilde{A}_{j_{k-1}}^{q_{1}} \tilde{A}_{j_{k}}^{\prime q_{0}} \tilde{C}_{i}^{\prime} \tilde{C}_{i} \tilde{A}_{i_{m}}^{p_{0}} \tilde{A}_{i_{m-1}}^{p_{1}} \cdots \tilde{A}_{j}^{p_{m}} x=0
$$

para quaisquer sequências de estados $j, i_{1}, \ldots, i_{m}, i$ e $j, j_{1}, \ldots, j_{k}, i$ tais que $\tilde{\lambda}_{j, i_{1}} \tilde{\lambda}_{i_{1}, i_{2}} \cdots \tilde{\lambda}_{i_{m}, i} \neq 0 \mathrm{e} / \mathrm{ou} \tilde{\lambda}_{j, j_{1}} \tilde{\lambda}_{j_{1}, j_{2}} \cdots \tilde{\lambda}_{j_{m}, i} \neq 0, \operatorname{com} p_{\ell}, q_{\nu} \geq 0, \ell=0, \ldots, m$ $\nu=0, \ldots, k$. Ao expandir $x^{\prime} O_{j}(k) x$ usando (3.4) obtemos somas de expressões como as do lado esquerdo de (3.13) (cada um de seus termos multiplicado por um produto de taxas de probabilidade), e desta forma (3.13) produz $x^{\prime} O_{j}(k) x=0$. 
É amplamente conhecido no caso de sistemas lineares determinísticos que o gramiano de alcançabilidade de $(A, B)$ é igual ao gramiano de observabilidade de $\left(A^{\prime}, B^{\prime}\right)$; nós usaremos este tipo de construção na seção a seguir visando explorar resultados de OM derivados nesta seção.

\subsection{Propriedades dos gramianos de alcançabilidade e con- trolabilidade de SLSM e relações de dualidade com a observabilidade}

Nesta seção retomamos o sistema (2.8). No caso particular "sem saltos" sendo $N=1, \tilde{\Lambda}=\Lambda=0$ e $\tilde{A}_{1}=A_{1}^{\prime}, \tilde{C}_{1}=B_{1}^{\prime}$, é bem sabido, considerando uma certa mudança de variável em relação ao tempo, que $\Upsilon_{\text {obs }}(0, t)=\Upsilon_{\text {rch }}(0, t)$ (possivelmente isto seja mais disseminado que a alcançabilidade de $\left(A_{1}, B_{1}\right)$ é equivalente à observabilidade de $\left(A_{1}^{\prime}, B_{1}^{\prime}\right)$, no sentido usual de sistemas determinísticos). Estendemos agora esta propriedade para SLSM. Consideraremos o sistema em (3.1) com o cenário

$$
\left\{\begin{array}{l}
\tilde{A}=A^{\prime}, \tilde{B}=0, \tilde{C}=B^{\prime}, \tilde{D}=0, \\
\tilde{\lambda}_{\kappa \ell}=\lambda_{\ell \kappa}, \text { para } \kappa \neq \ell, \text { e } \tilde{\lambda}_{\ell \ell}=-\sum_{\kappa \neq \ell} \tilde{\lambda}_{\ell \kappa} .
\end{array}\right.
$$

Lema 3. Considere o sistema (2.8) e o sistema (3.1) com os parâmetros em (3.14). Então, para cada realização da cadeia de Markov começando em i e visitando $j$ no tempo $t \geq 0$, com $i, j \in \mathcal{S}_{\pi}$ sendo estados de Markov comunicantes, temos

$$
\Upsilon_{\text {rch }}(0, t)=\Upsilon_{\text {obs }}(0, t)
$$

Demonstração. Consideremos a sequência $j=: i_{0}, i_{1}, \ldots, i_{m-1}, i_{m}:=i$ tal que $\tilde{\lambda}_{j, i_{1}} \tilde{\lambda}_{i_{1}, i_{2}} \cdots \tilde{\lambda}_{i_{m-1}, i} \neq 0$ com sua respectiva sequência de tempos de salto $0 \leq$ $s_{1} \leq \ldots \leq s_{m} \leq t$. Ao denotar $s_{0}:=0$ e $s_{m+1}:=t$, temos,

$$
\begin{aligned}
\Upsilon_{\text {obs }}(0, t)= & \sum_{k=0}^{m} \int_{s_{k}}^{s_{k+1}} \tilde{\Phi}(s, 0)^{\prime} \tilde{C}_{i_{k}}^{\prime} \tilde{C}_{i_{k}} \tilde{\Phi}(s, 0) d s=\int_{0}^{s_{1}} e^{\tilde{A}_{j}^{\prime} s} \tilde{C}_{j}^{\prime} \tilde{C}_{j} e^{\tilde{A}_{j} s} d s \\
& +\ldots+e^{\tilde{A}_{j}^{\prime} s_{1}} \cdots e^{\tilde{A}_{i_{m-1}}^{\prime}\left(s_{m}-s_{m-1}\right)}\left[\int_{s_{m}}^{t} e^{\tilde{A}_{i}^{\prime}\left(s-s_{m}\right)} \tilde{C}_{i}^{\prime} \tilde{C}_{i} e^{\tilde{A}_{i}\left(s-s_{m}\right)} d s\right] \\
& \times e^{\tilde{A}_{i_{m-1}}\left(s_{m}-s_{m-1}\right)} \cdots e^{\tilde{A}_{j} s_{1}} .
\end{aligned}
$$


Definamos $t_{0}, \ldots, t_{m+1}$ satisfazendo $0=t_{0} \leq\left(t-s_{m}=t_{1}\right) \leq \ldots \leq\left(t-s_{1}=\right.$ $\left.t_{m}\right) \leq t=t_{m+1}$. Desde que $\tilde{A}=A^{\prime}, \tilde{C}=B^{\prime}, \tilde{B}=0, \tilde{D}=0$ e a matriz de transição $\tilde{\Lambda}$ é tal que $\tilde{\lambda}_{\kappa \ell}=\lambda_{\ell \kappa}$, para $\kappa \neq \ell$, obtemos de (3.15) com $\tau=t-s$ :

$$
\begin{aligned}
\Upsilon_{\mathrm{obs}}(0, t)= & e^{A_{i_{j}}\left(t-t_{m}\right)} \cdots e^{A_{i_{m-1}}\left(t_{2}-t_{1}\right)}\left[\int_{0}^{t_{1}} e^{A_{i}\left(t_{1}-\tau\right)} B_{i} B_{i}^{\prime} e^{A_{i}^{\prime}\left(t_{1}-\tau\right)} d \tau\right] \\
& \times e^{A_{i_{m-1}}^{\prime}\left(t_{2}-t_{1}\right)} \cdots e^{A_{j}^{\prime}\left(t-t_{m}\right)}+\ldots+\int_{t_{m}}^{t} e^{A_{j}(t-\tau)} B_{j} B_{j}^{\prime} e^{A_{j}^{\prime}(t-\tau)} d \tau \\
= & \sum_{k=0}^{m} \int_{t_{k}}^{t_{k+1}} \Phi(t, \tau) B_{i_{m-k}} B_{i_{m-k}}^{\prime} \Phi(t, \tau)^{\prime} d \tau=\Upsilon_{\mathrm{rch}}(0, t),
\end{aligned}
$$

sendo $\lambda_{i, i_{m-1}} \cdots \lambda_{i_{1}, j} \neq 0$.

Precisamos comparar o conjunto $\left\{x: W^{t}(x, j)=0\right\}$ com o espaço nulo de $\mathcal{E}\left\{\Upsilon_{\mathrm{rch}}(0, t) \mid \theta(0)=i\right\}$. Uma dificuldade é que as condições iniciais da cadeia de Markov poderiam ser diferentes, $i \neq j$, de tal maneira que o Lema 3 por si só não responde a esta incógnita.

Proposição 7. Suponha a hipótese do Lema 3. Para $t>0$ dado e cada $x \in \mathbb{R}^{n}$ e $j \in \mathcal{S}_{\pi}$, temos que $W^{t}(x, j)=0$ é equivalente a $x^{\prime} \mathcal{E}\left\{\Upsilon_{\mathrm{rch}}(0, t) \mid \theta(0)=i\right\} x=0$ sempre que $i$ e $j$ sejam estados comunicantes.

Demonstração. Apresentamos a prova da necessidade somente, pois a suficiência é muito similar. Assumimos $x^{\prime} \mathcal{E}\left\{\Upsilon_{\mathrm{rch}}(0, t) \mid \theta(0)=i\right\} x=0$, sendo $j$ alcançado por $i$. Podemos verificar para cada $t_{f} \geq 0$ que $x^{\prime} \mathcal{E}\left\{\Upsilon_{\text {rch }}\left(0, t_{f}\right) \mid \theta(0)=\right.$ $i\} x=0$. Isto leva em $x^{\prime} \mathcal{E}\left\{\Upsilon_{\text {rch }}\left(0, t_{f}\right) \mid \theta(0)=i, \theta\left(t_{f}\right)=j\right\} x=0$, o qual pelo Lema 3 é equivalente a $x^{\prime} \mathcal{E}\left\{\Upsilon_{\text {obs }}\left(0, t_{f}\right) \mid \tilde{\theta}(0)=j, \tilde{\theta}\left(t_{f}\right)=i\right\} x=0$. Isto e a desigualdade $\Upsilon_{\text {obs }}\left(0, t_{f}\right) \geq \Upsilon_{\text {obs }}(0, t)$ (quando $t_{f} \geq t$ ) produzem $x^{\prime} \mathcal{E}\left\{\Upsilon_{\text {obs }}(0, t) \mid \tilde{\theta}(0)=\right.$ $\left.j, \tilde{\theta}\left(t_{f}\right)=i\right\} x=0$. Desde que $i, j$ são comunicantes, qualquer $\theta(t)$ que possa ser alcançado por $\theta(0)=j$ pode pela sua vez alcançar $\theta\left(t_{f}\right)=i$, o qual permite concluir que a informação $\tilde{\theta}\left(t_{f}\right)=i$ é irrelevante quando é calculado o último valor esperado, implicando que $x^{\prime} \mathcal{E}\left\{\Upsilon_{\text {obs }}(0, t) \mid \tilde{\theta}(0)=j\right\} x=0$ e de (3.3), $W(x, j)=0$.

Consideremos a sequência de coleção de matrizes $R(k) \in \mathcal{M}^{n}, k \in \mathbb{Z}^{0}$, satisfazendo

$$
\begin{aligned}
R_{j}(k+1) & =\mathcal{T}_{A, j}(R(k)), \quad k \in \mathbb{Z}^{0}, j \in \mathcal{S}, \\
R_{j}(0) & =1_{\left\{j \in \mathcal{S}_{\pi}\right\}} B_{j} B_{j}^{\prime} .
\end{aligned}
$$

$R(k)$ pode ser interpretada como um dual de $O(k)$ definida em (3.4) e cumprirá um importante papel no teste computacional para AM. O Lema a seguir faz 
uma extensão dos resultados da Proposição 6 ao sistema original, levando em uma propriedade de invariância para as matrizes $B_{i}$.

Lema 4. Considere o sistema $(A, B, C=0, D=0, \Lambda)$. Para cada $x \in \mathbb{R}^{n} e$ $i, j \in \mathcal{S}_{\pi}$ estados comunicantes de estados de Markov, as seguintes afirmações são equivalentes:

(i) $x^{\prime} \mathcal{E}\left\{\Upsilon_{\mathrm{rch}}(0, t) \mid \theta(0)=i\right\} x=0, t \geq 0$.

(ii) $x^{\prime} R_{j}(k) x=0, k \in \mathbb{Z}^{0}$.

(iii) $B_{i}^{\prime} A_{\iota_{1}}^{p_{0}} A_{\iota_{2}}^{p_{1}} \cdots A_{j}^{p_{m}} x=0$, para cada sequência de estados de Markov $i, \iota_{1}, \ldots, \iota_{m}, j$ contida em $\mathcal{S}_{\pi}$ tal que $\lambda_{i, \iota_{1}} \lambda_{\iota_{1}, \iota_{2}} \cdots \lambda_{\iota_{m}, j} \neq 0$ com $p_{\ell} \geq 0$, $\ell=0, \ldots, m$.

Demonstração. $(i) \Leftrightarrow($ iii $)$. Considerando o sistema (3.1) com os parâmetros em (3.14), concluímos que a afirmação $(i)$ da Proposição 6 é válida. Consequentemente temos que (iii) da Proposição 6 é válida e, pela sua vez, pode ser rescrita como (iii) acima. $(i i i) \Rightarrow(i i)$. Similar à prova da implicação $($ iii $) \Rightarrow($ ii $)$ da proposição 6 . $(i i) \Rightarrow(i)$. Considerando o sistema (3.1) com os parâmetros em (3.14), usando (3.17) podemos escrever

$$
\begin{aligned}
R_{j}(k+1) & =\tilde{A}_{j}^{\prime} R_{j}(k)+R_{j}(k) \tilde{A}_{j}+\sum_{i \in \mathcal{S}} \tilde{\lambda}_{j i} R_{i}(k)+\lambda_{j j} R_{j}(k)-\tilde{\lambda}_{j j} R_{j}(k) \\
& =\bar{A}_{j}^{\prime} R_{j}(k)+R_{j}(k) \bar{A}_{j}+\sum_{i \in \mathcal{S}} \tilde{\lambda}_{j i} R_{i}(k) \\
& =\mathcal{L}_{\bar{A}, j}(R(k))
\end{aligned}
$$

sendo $\bar{A}_{j}=\tilde{A}_{j}+\kappa_{j} I$, com $\kappa_{j}=\frac{1}{2}\left(\lambda_{j j}-\tilde{\lambda}_{j j}\right)=\frac{1}{2} \sum_{i \in \mathcal{S}} \lambda_{i j}$. Isto significa que $R(k)$ satisfaz (3.4) (com a matriz $\tilde{A}$ substituída com $\bar{A}$ ), e podemos usar a Proposição 6 para concluir que

$$
\tilde{C}_{i} \bar{A}_{i_{m}}^{p_{0}} \bar{A}_{i_{m-1}}^{p_{1}} \cdots \bar{A}_{j}^{p_{m}} x=0
$$

para quaisquer estados de Markov $j, i_{1}, \ldots, i_{m}, i$ tais que $\tilde{\lambda}_{j, i_{1}} \tilde{\lambda}_{i_{1}, i_{2}} \cdots \tilde{\lambda}_{i_{m}, i} \neq$ 0 com $p_{\ell} \geq 0, \ell=0, \ldots, m$. Expandindo a expressão $\tilde{C}_{i} \tilde{A}_{i_{m}}^{q_{0}} \tilde{A}_{i_{m-1}}^{q_{1}} \cdots \tilde{A}_{j}^{q_{m}} x \mathrm{com}$ $q_{\ell} \geq 0, \ell=0, \ldots, m$, ao substituir $\tilde{A}_{j}=\bar{A}_{j}-\kappa_{j} I$ para cada $j \in \mathcal{S}_{\pi}$, obtemos uma soma de expressões exatamente como no lado esquerdo de (3.19), cada uma das quais sendo igual a zero. Isto produz $\tilde{C}_{i} \tilde{A}_{i_{m}}^{q_{0}} \tilde{A}_{i_{m-1}}^{q_{1}} \cdots \tilde{A}_{j}^{q_{m}} x=0$, e da Proposição 6 temos $W^{t}(x, j)=0$. O resultado segue diretamente pela Proposição 7. 
Corolário 3. Considere $j \in \mathcal{S}_{\pi}$ e $v \in \mathbb{R}^{n}$. Então, para todo $k \in \mathbb{Z}^{0}$,

$$
v^{\prime} R_{j}(k) v=0 \quad \text { é equivalente a } \quad R_{j}(k) v=0 .
$$

Demonstração. A implicação $(\Leftarrow)$ é trivial e a contrária pode ser obtida usando a equivalência em $($ ii $)$ e (iii) do Lema 4.

Em analogia com a observabilidade é possível também obter resultados de invariância envolvendo o gramiano de controlabilidade. Consideremos agora o sistema em (3.1) com o cenário

$$
\tilde{A}=-A^{\prime}, \tilde{B}=0, \tilde{C}=B^{\prime}, \tilde{D}=0, \tilde{\Lambda}=\Lambda \text {. }
$$

De fato, assumindo (3.20) e dado $t>0$, para qualquer realização da cadeia até o instante $\tau \in[0, t]$, visitando os estados arbitrários $i, i_{1}, \ldots, i_{q}$ com correspondentes tempos de salto, $t_{1}, \ldots, t_{q}$, temos:

$$
\begin{aligned}
\Phi(0, \tau) & =\left[\Phi\left(\tau, t_{q}\right) \Phi\left(t_{q}, t_{q-1}\right) \cdots \Phi\left(t_{1}, 0\right)\right]^{-1} \\
& =\left(\left[\Phi^{-1}\left(\tau, t_{q}\right)\right]^{\prime}\left[\Phi^{-1}\left(t_{q}, t_{q-1}\right)\right]^{\prime} \cdots\left[\Phi^{-1}\left(t_{1}, 0\right)\right]^{\prime}\right)^{\prime} \\
& =\left[e^{-A_{i_{q}}^{\prime}\left(\tau-t_{q}\right)} e^{-A_{i_{q-1}}^{\prime}\left(t_{q}-t_{q-1}\right)} \cdots e^{-A_{i}^{\prime} t_{1}}\right]^{\prime} \\
& =\tilde{\Phi}(\tau, 0)^{\prime} .
\end{aligned}
$$

Portanto, de (3.21) temos $\Upsilon_{\text {obs }}(0, t)=\Upsilon_{\text {cnt }}(0, t)$, o que nos leva de maneira direta no seguinte resultado.

Lema 5. Considere o sistema (2.8) e o sistema (3.1) com os parâmetros em (3.20). Então, para um dado $t>0$ e cada $x \in \mathbb{R}^{n}$ e $i \in \mathcal{S}_{\pi}$, temos que $W^{t}(x, i)=0$ é equivalente a $x^{\prime} \mathcal{E}\left\{\Upsilon_{\mathrm{cnt}}(0, t) \mid \theta(0)=i\right\} x=0$.

Seja agora $\mathcal{C}(k) \in \mathcal{M}^{n 0}$ definida recursivamente para $k \in \mathbb{Z}^{0}$ como:

$$
\begin{aligned}
\mathcal{C}_{i}(k+1) & =\mathcal{H}_{A, i}(\mathcal{C}(k)), \quad k \in \mathbb{Z}^{0}, i \in \mathcal{S}_{\pi} \\
\mathcal{C}_{i}(0) & =B_{i} B_{i}^{\prime} .
\end{aligned}
$$

Observe-se que a definição da sequência $\mathcal{C}(k), k \in \mathbb{Z}^{0}$ de coleção de matrizes é baseada no operador $\mathcal{H}_{A}$ sendo que o sinal negativo envolvido nesta recursão mantem a compatibilidade com a definição das sequências $O(k)$ e $R(k), k \in$ $\mathbb{Z}^{0}$. Este sinal negativo obedece à configuração em (3.20), a qual é usada no resultado que segue: as propriedades na Proposição 6 serão adaptadas ao 
sistema em (2.8) para obter propriedades de invariância associadas ao gramiano de controlabilidade.

Lema 6. Consideremos o sistema $(A, B, C=0, D=0, \Lambda)$. Para cada $x \in \mathbb{R}^{n}$, as seguintes afirmações são equivalentes:

(i) $x^{\prime} \mathcal{E}\left\{\Upsilon_{\mathrm{cnt}}(0, t) \mid \theta(0)=i\right\} x=0, t \geq 0, i \in \mathcal{S}_{\pi}$.

(ii) $x^{\prime} \mathcal{C}_{i}(k) x=0, k \in \mathbb{Z}^{0}, i \in \mathcal{S}_{\pi}$.

(iii) $B_{j} A_{j_{m}}^{p_{0}} A_{j_{m-1}}^{p_{1}} \cdots \tilde{A}_{i}^{p_{m}} x=0$, para qualquer sequência de estados de Markov $i, j_{1}, \ldots, j_{m}, j$ contida em $\mathcal{S}_{\pi}$ tal que $\lambda_{i, j_{1}} \lambda_{j_{1}, j_{2}} \cdots \lambda_{j_{m}, j} \neq 0$ com $p_{\ell} \geq 0$, $\ell=0, \ldots, m$.

Demonstração. $(i) \Leftrightarrow($ iii). Usando (3.20), a prova é similar à da implicação $(i) \Leftrightarrow($ iii $)$ no Lema 4. (i) $\Leftrightarrow($ ii). Considerando o sistema em (3.1) com os parâmetros em (3.20), notamos que (3.22) pode ser escrita como:

$$
\begin{aligned}
\mathcal{C}_{i}(k+1) & =\tilde{A}_{i}^{\prime} \mathcal{C}_{i}(k)+\mathcal{C}_{i}(k) \tilde{A}_{i}+\sum_{j \in \mathcal{S}} \tilde{\lambda}_{i j} \mathcal{C}_{i}(k) \\
& =\mathcal{L}_{\tilde{A}, i}(\mathcal{C}(k))
\end{aligned}
$$

com $\mathcal{C}_{i}(0)=\tilde{C}_{i}^{\prime} \tilde{C}_{i}$. Isto significa que $\mathcal{C}(k)$ é dada pela recursão em (3.4); Portanto, de (3.23) e Proposição 6 temos que $x^{\prime} \mathcal{C}_{i}(k) x=0$ é equivalente a $W^{t}(x, i)=0$. O Lema 5 leva no resultado.

Corolário 4. Considere $i \in \mathcal{S}_{\pi} e v \in \mathbb{R}^{n}$. Então, para todo $k \in \mathbb{Z}^{0}$,

$$
v^{\prime} \mathcal{C}_{i}(k) v=0 \quad \text { é equivalente a } \quad \mathcal{C}_{i}(k) v=0 .
$$

Demonstração. A implicação $(\Leftarrow)$ é trivial e a contrária pode ser obtida usando a equivalência em $($ ii $)$ e (iii) do Lema 6. 


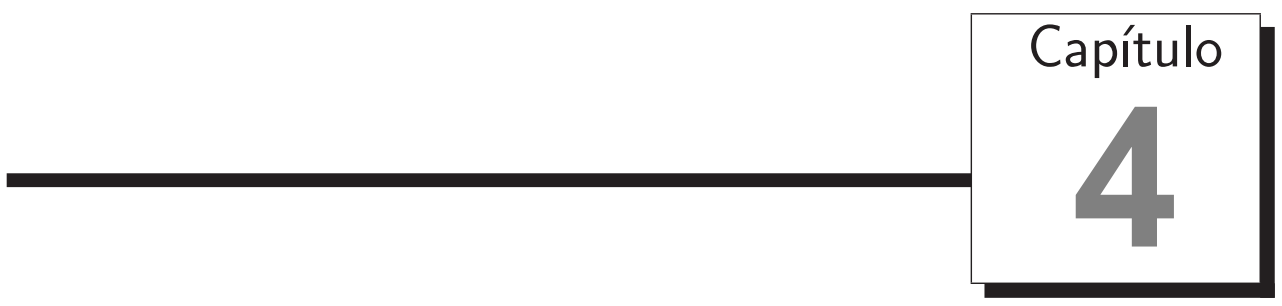

\section{Alcançabilidade média e um teste computacional}

Vamos considerar duas noções de AM exigindo a positividade do gramiano em diversos sentidos: AM requer positividade para qualquer distribuição inicial $\pi$ da cadeia de Markov; $\pi$-AM considera uma distribuição inicial particular $\pi$ dada. Nós também devotamos uma pequena parte deste trabalho a uma terceira noção, chamada П-AM, a qual assume $\pi \in \Pi$, sendo $\Pi$ um conjunto de distribuições tais que os estados transientes nunca são visitados.

\subsection{Alcançabilidade média sujeita a $\pi$}

Quando procuramos condições para a positividade da esperança do segundo momento de $x$, é importante notar que esta quantidade depende não somente da estrutura de $A$ e $\Lambda$ mas também da distribuição de probabilidade inicial $\pi$, já que os estados de Markov que são visitados estão "selecionados" essencialmente por $\pi$ e $\Lambda$, como ilustrado no seguinte exemplo.

Exemplo 2. Consideremos o sistema $(A, B, \Lambda) \operatorname{com} A_{1}=A_{2}=B_{2}=0$, $B_{1}=1, \Lambda=0$ e $x_{0}=0$; suponha que a dinâmica da componente contínua do estado é perturbada por um processo de Wiener com operador covariância incremental Idt. Para qualquer $0 \leq \alpha \leq 1$ temos $E\left\{x(t) x^{\prime}(t) \mid \pi=[\alpha 1-\alpha]\right\}=$ $\alpha E\left\{w(t) w^{\prime}(t)\right\}=\alpha$. Então $E\left\{x(t) x(t)^{\prime} \mid \pi\right\}>0$ se e somente se $\alpha \neq 0$. 
Definição 8 ( $\pi$-AM). A terna $(A, B, \Lambda)$ é alcançável em média em relação a $\pi(\pi-A M)$ se existe $t>0$ tal que $\mathcal{E}\left\{\Upsilon_{\text {rch }}(0, t) \mid \theta(0)=i\right\}>0$, para todo $i \in \mathcal{S}_{\pi}$.

Observação 2. Um raciocínio similar como aquele da prova de $(i i) \Rightarrow(i)$ do Lema 4 permite concluir que $\pi$-AM é invariante a perturbações da forma $\kappa_{j} I$ na matriz $A_{j}$, com $\kappa_{j} \in \mathbb{R}$. Em particular $(A, B, \Lambda)$ é $\pi-A M$ se o somente se $(A+\kappa I, B, \Lambda)$ é $\pi-A M$.

Introduzimos na continuação uma equação diferencial ordinária (EDO) bastante comum cuja solução $S_{j}(t), j \in \mathcal{S}, t \geq 0$, está relacionada com o gramiano de alcançabilidade via $R$ definido em (3.17) e o Lema 7. $S(t)$ satisfaz

$$
\dot{S}_{j}(t)=\mathcal{T}_{A, j}(S(t))+1_{\left\{j \in \mathcal{S}_{\pi}\right\}} B_{j} B_{j}^{\prime}, \quad S_{j}(0)=0, j \in \mathcal{S} .
$$

A facilidade para manipular a solução exponencial de (4.1) é útil no que segue. A interpretação para $S$ será adiada para a Seção 5.1 quando seja estudado a sua relação com o segundo momento condicional $X_{i}=\mathcal{E}\left\{x(t) x(t) 1_{\{\theta=i\}}\right\}$. Usando os operadores vec e $\varphi$, podemos vetorizar (4.1) para obter um sistema linear estândar invariante no tempo da forma $\dot{\sigma}(t)=\mathcal{A}^{\prime} \sigma(t)+b$ sendo $\mathcal{A}$ como em $(2.11)$ e $b=\varphi\left(B_{\pi} B_{\pi}^{\prime}\right)$, sendo $B_{\pi} B_{\pi}^{\prime}$ a coleção formada pelas matrizes $1_{\left\{j \in \mathcal{S}_{\pi}\right\}} B_{j} B_{j}^{\prime}$. Notando que existem $n(n-1) N / 2$ entradas repetidas, tanto em $\sigma$ quanto em $b$ (devido à simetria de $S_{j}(t), \mathcal{T}_{A}(\cdot)$ e $B_{j} B_{j}^{\prime}$ em $(4.1)$ ), estabelecemos $\bar{N}:=n^{2} N-\frac{n(n-1) N}{2}=\frac{n(n+1) N}{2}$ e consideramos a matriz de projeção $\mathfrak{P} \in$ $\mathfrak{R}^{n^{2} N, \bar{N}}$ que omite estas entradas. Definimos $\hat{\sigma} \in \mathbb{R}^{\bar{N}}, \hat{b} \in \mathbb{R}^{\bar{N}}$ e $\hat{\mathcal{A}} \in \mathfrak{R}^{\bar{N}, \bar{N}}$ dado como $\hat{\sigma}=\mathfrak{P} \sigma, \hat{b}=\mathfrak{P} b$ e $\hat{\mathcal{A}}=\mathfrak{P}^{\prime} \mathcal{A} \mathfrak{P}$ respectivamente, levando no sistema de ordem reduzida

$$
\dot{\hat{\sigma}}(t)=\hat{\mathcal{A}}^{\prime} \hat{\sigma}(t)+\hat{b}, \quad \hat{\sigma}(0)=0 .
$$

Note que as derivadas de ordem $k, k \geq 1$, de $\hat{\sigma}(t)$ calculadas em $t=0$ são dadas por:

$$
\frac{d^{k} \hat{\sigma}}{d t^{k}}(0)=\hat{\mathcal{A}}^{\prime k-1} \hat{b}
$$

O seguinte resultado fornece a relação entre a função $S$ e as suas derivadas.

Lema 7. Seja $j \in \mathcal{S}_{\pi, \text { rec }}$ e $v \in \mathbb{R}^{n}$. Defina $V=\left(V_{1}, \ldots, V_{N}\right)$ tal que $V_{j}=v v^{\prime}$ e, se $i \neq j, V_{i}=0$ e, $\hat{w}=\mathfrak{P} \varphi(V)$. As seguintes afirmações são equivalentes:

(i) $v^{\prime} S_{j}(s) v=0$ ou, equivalentemente, $\hat{w}^{\prime} \hat{\sigma}(s)=0$ para algum $s>0$.

(ii) $\hat{w}^{\prime} \frac{d^{k+1} \hat{\sigma}}{d t^{k+1}}(0)=0$, para $k \in \mathbb{Z}^{0}$ 
(iii) $v^{\prime} S_{j}(t) v=0$ ou, equivalentemente, $\hat{w}^{\prime} \hat{\sigma}(t)=0$ para todo $t \geq 0$.

(iv) $v^{\prime} \mathcal{E}\left\{\Upsilon_{r c h}(0, t) \mid \theta(0)=i\right\} v=0$, para todo $i \in \mathcal{S}_{\pi}$ comunicante com $j$ e todo $t \geq 0$.

Demonstração. $(i) \Rightarrow(i i)$. Suponha que existe $s>0$ tal que $v^{\prime} S_{j}(s) v=0$. Então,

$$
\begin{aligned}
\hat{w}^{\prime} \hat{\sigma}(s)=0 & \Rightarrow \hat{w}^{\prime} \int_{0}^{s} e^{\hat{\mathcal{A}}^{\prime}(s-\tau)} \hat{b} d \tau=0 \Rightarrow \hat{w}^{\prime} e^{\hat{\mathcal{A}}^{\prime}(s-\tau)} \hat{b}=0, \quad 0 \leq \tau \leq s \\
& \Rightarrow \hat{w}^{\prime} \hat{\mathcal{A}}^{\prime k} e^{\hat{\mathcal{A}}^{\prime}(s-\tau)} \hat{b}=0, \quad 0 \leq \tau \leq s
\end{aligned}
$$

Em particular para $\tau=s$ temos $\hat{w}^{\prime} \hat{\mathcal{A}}^{\prime k} \hat{b}=0$, ou, melhor, $\hat{w}^{\prime} \frac{d^{k+1} \hat{\sigma}}{d t^{k+1}}(0)=0$, para todo $k=0,1, \ldots$ Note que $e^{\hat{\mathcal{A}}^{\prime}(s-\tau)} \hat{b}=\mathfrak{P} \varphi\left(S_{B_{\pi}}(t)\right)$, sendo $S_{B_{\pi}}$ uma coleção de funções matriciais definida de uma maneira tal que satisfaz $\varphi\left(\dot{S}_{B_{\pi}}(t)\right)=$ $\mathcal{A}^{\prime} \varphi\left(S_{B_{\pi}}(t)\right)$, com $S_{B_{\pi}}(0)=B_{\pi} B_{\pi}^{\prime}$. Assim, $S_{B_{\pi}} \in \mathcal{M}^{n 0}$ e daqui $v^{\prime} S_{B_{\pi}, j}(s-$ $\tau) v=\left\langle V, S_{B_{\pi}}(s-\tau)\right\rangle \geq 0$, implicando em $\hat{w}^{\prime} e^{\hat{\mathcal{A}}^{\prime}(s-\tau)} \hat{b} \geq 0$ o qual nos leva na segunda implicação em (4.4).

$($ ii $) \Rightarrow($ iii $)$. Usando a expansão em série de potências obtemos $e^{\hat{\mathcal{A}}^{\prime}(t-\tau)}=$ $\sum_{k=0}^{\infty} \hat{\alpha}_{k}(t-\tau) \hat{\mathcal{A}}^{\prime k}$, sendo $\hat{\alpha}_{k}(t-\tau)=\frac{(t-\tau)^{k}}{k !}$ para cada $k \in \mathbb{Z}^{0}$. Então,

$$
\hat{w}^{\prime} \hat{\sigma}(t)=\int_{0}^{t} \hat{w}^{\prime} \sum_{k=0}^{\infty} \hat{\alpha}_{k}(t-\tau) \hat{\mathcal{A}}^{\prime k} \hat{b} d \tau=\sum_{k=0}^{\infty}\left(\int_{0}^{t} \hat{\alpha}_{k}(t-\tau) d \tau\right) \hat{w}^{\prime} \hat{\mathcal{A}}^{\prime k} \hat{b}
$$

e, já que (4.3) e (ii) ambas produzem $\hat{w}^{\prime} \hat{\mathcal{A}}^{\prime k} \hat{b}=0$, obtemos $\langle S(t), V\rangle=\hat{w}^{\prime} \hat{\sigma}(t)=$ 0 . Equivalentemente $v^{\prime} S_{j}(t) v=0$, para todo $t \geq 0$.

$($ iii $) \Rightarrow(i)$. Trivial.

$($ iii $) \Leftrightarrow(i v)$. Segue do Lema 4 e a equivalência entre (ii) e (iii) acima.

Podemos agora usar o Lema 7 no estudo do espaço nulo de $R$ e seu papel ao testar $\pi$-AM. O enlace entre a função $S$ e $R$ segue diretamente de (3.17) e (4.1):

$$
R_{j}(k)=\frac{d^{k+1} S_{j}}{d t^{k+1}}(0), \quad k \in \mathbb{Z}^{0}, j \in \mathcal{S} .
$$

Para facilitar a notação, vamos definir as matrizes de alcançabilidade $\mathbf{R}_{j} \in$ $\mathfrak{R}^{n, n \bar{N}}$ por:

$$
\mathbf{R}_{j}=\left[\begin{array}{llll}
R_{j}(0) & R_{j}(1) & \ldots & R_{j}(\bar{N}-1)
\end{array}\right],
$$

para todo $j \in \mathcal{S}_{\pi, \text { rec }}$ Escrevemos $\mathbf{R}_{j}(A, B, \Lambda, \pi)$ em algumas passagens para enfatizar os parâmetros requeridos para calcular $\mathbf{R}_{j}$. 
Proposição 8. Seja $j \in \mathcal{S}_{\pi, \text { rec }}$. Temos que $v \in \mathcal{N}\left(R_{j}(k)\right), k=0, \ldots, \bar{N}-1$, é equivalente a $v \in \mathcal{N}\left(R_{j}(k)\right), k=0, \ldots, m$, para $m \geq \bar{N}$.

Demonstração. Vamos mostrar só umas das implicações já que a inversa é trivial.Da hipótese e (4.6) segue que $v^{\prime} \frac{d^{k+1} S_{j}}{d t^{k+1}}(0) v=0, k=0, \ldots, \bar{N}-1$, o qual é equivalente a $w^{\prime} \hat{\mathcal{A}}^{\prime \prime} \hat{b}=0, k=0, \ldots, \bar{N}-1$, sendo $\hat{w}$ definido como no Lema 7. Usando o Teorema de Cayley-Hamilton obtemos $w^{\prime} \hat{\mathcal{A}}^{\prime m} \hat{b}=0$, para $m \geq \bar{N}$. Isto é, $v^{\prime} R_{j}(m) v=0$, para $m \geq \bar{N}$. Corolário 3 completa a prova.

Proposição 9. $\mathcal{I} m\left(\mathbf{R}_{j}\right)=\mathcal{I} m\left(\left[R_{j}(0) R_{j}(1) \ldots R_{j}(m)\right]\right)$, para todo $m \geq \bar{N}$.

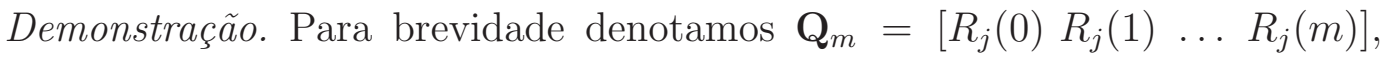
$m \geq \bar{N}$. Assuma $v \in \mathcal{I m}\left(\mathbf{Q}_{m}\right)$, tal que $v=\mathbf{Q}_{m} \xi$ para algum $\xi \in \mathbb{R}^{n(m+1)}$. Agora, tomamos um $\eta \in \mathcal{N}\left(\mathbf{R}_{j}^{\prime}\right)$ arbitrário e, pela definição de $\mathbf{R}_{j}$ temos $R_{j}(k) \eta=0, k=0, \ldots, \bar{N}-1$. Então, a Proposição 8 produz $R_{j}(k) \eta=0$, $k=0, \ldots, m$, sendo $m \geq \bar{N}$, levando em $\eta^{\prime} \mathbf{Q}_{m}=0$ e $\eta^{\prime} v=0$; em consequência $v \in\left(\mathcal{N}\left(\mathbf{R}_{j}^{\prime}\right)\right)^{\perp}$. Do teorema fundamental da álgebra linear temos que $v \in$ $\operatorname{Im}\left(\mathbf{R}_{j}\right)$. A implicação inversa pode ser mostrada de uma forma similar.

Proposição 10. Para quaisquer $i$ e $j$ estados de Markov comunicantes em $\mathcal{S}_{\pi}$ temos,

$$
\mathcal{I} m\left(\mathcal{E}\left\{\Upsilon_{\mathrm{rch}}(0, t) \mid \theta(0)=i\right\}\right)=\mathcal{I} m\left(\mathbf{R}_{j}\right)
$$

Demonstração. Assumimos $v \in \mathcal{I} m\left(\mathbf{R}_{j}\right)$. Então existe $\xi \in \mathbb{R}^{n \bar{N}}$ tal que $v=\mathbf{R}_{j} \xi$. Mais ainda, para qualquer $\eta \in \mathcal{N}\left(\mathcal{E}\left\{\Upsilon_{\text {rch }}(0, t) \mid \theta(0)=i\right\}\right.$, o Lema 7 implica em $\eta^{\prime} S_{j}(t) \eta=0$. Do Lema 7 e o Corolário 3 obtemos $\eta^{\prime} R_{j}(k)=0$, $k=0, \ldots, \bar{N}-1$. Consequentemente, temos que $\eta^{\prime} \mathbf{R}_{j}=0$ e de aqui $\eta^{\prime} \mathbf{R}_{j} \xi=0$, levando pela sua vez em $\eta^{\prime} v=0$. Assim, pelo fato de que $\eta$ é arbitrário em $\mathcal{N}\left(\mathcal{E}\left\{\Upsilon_{\text {rch }}(0, t) \mid \theta(0)=i\right\}\right.$ concluímos que $v \in\left(\mathcal{N}\left(\mathcal{E}\left\{\Upsilon_{\text {rch }}(0, t) \mid \theta(t)=i\right\}\right)\right)^{\perp}$. Portanto, segundo o teorema fundamental da álgebra linear obtemos $v \in$ $\operatorname{Im}\left(\mathcal{E}\left\{\Upsilon_{\text {rch }}(0, t) \mid \theta(0)=i\right\}\right)$. A implicação recíproca pode ser mostrada de uma maneira similar, substituindo $\mathbf{R}_{j}$ por $\mathcal{E}\left\{\Upsilon_{\text {rch }}(0, t) \mid \theta(0)=i\right\}$ (e vice-versa) na prova acima.

Teorema 1. $(A, B, \Lambda)$ é $\pi-A M$ se e somente se $\operatorname{posto}\left(\mathbf{R}_{j}\right)=n$, para todo $j \in \mathcal{S}_{\pi, \text { rec }}$.

Demonstração. Necessidade. É imediato da definição de $\pi$-AM e a Proposição 10 que $\mathcal{I} m\left(\mathbf{R}_{j}\right)=\mathbb{R}^{n}$ para qualquer $j$ tal que $i, j$ sejam estados comunicantes 
e $i \in \mathcal{S}_{\pi}$. Isto produz $\operatorname{posto}\left(\mathbf{R}_{j}\right)=n, j \in \mathcal{S}_{\pi, \text { rec }}$. Suficiência. Desde que $\operatorname{posto}\left(\mathbf{R}_{j}\right)=n$ temos da Proposição 10 que

$$
\mathcal{E}\left\{\Upsilon_{\text {rch }}(0, \bar{t}) \mid \theta(0)=i\right\}>0
$$

para qualquer $\bar{t}>0$ e $i \in \mathcal{S}_{\pi}$ comunicante com $j$. Só resta mostrar a positividade do gramiano para estados de Markov iniciais que não se comunicam com $j$, isto é, para estados transientes $\theta(0)=\ell$ que alcançam $j$. Seja $T^{*}$ o primeiro tempo de visita à classe recorrente $\mathcal{S}_{\pi \text {,rec }}$ que contem $j$, e seja $s \geq 0$. Então,

$$
\begin{aligned}
\mathcal{E}\left\{\Upsilon_{\mathrm{rch}}(0, \bar{t}+s) \mid \theta(0)=\ell\right\}=\mathcal{E}\left\{\Upsilon_{\mathrm{rch}}(0, \bar{t}+s) \mid \theta(0)=\ell, T^{*} \leq s\right\} \\
\quad \times \mathcal{P}\left(T^{*} \leq s \mid \theta(0)=\ell\right)+\mathcal{E}\left\{\Upsilon_{\mathrm{rch}}(0, \bar{t}+s) \mid \theta(0)=\ell, T^{*}>s\right\} \\
\times \mathcal{P}\left(T^{*}>s \mid \theta(0)=\ell\right) .
\end{aligned}
$$

É evidente que $\mathcal{P}\left(T^{*} \leq s \mid \theta(0)=\ell\right)>0$; Ademais, $\mathcal{E}\left\{\Upsilon_{\mathrm{rch}}(0, \bar{t}+s) \mid \theta(0)=\right.$ $\left.\ell, T^{*} \leq s\right\} \geq \mathcal{E}\left\{\Upsilon_{\mathrm{rch}}\left(T^{*}, \bar{t}+s\right) \mid \theta(0)=\ell, T^{*} \leq s\right\}=\mathcal{E}\left\{\Upsilon_{\mathrm{rch}}\left(T^{*}, \bar{t}+s\right) \mid \theta\left(T^{*}\right)=\right.$ $\left.i \in \mathcal{S}_{\pi, \text { rec }}\right\} \geq \mathcal{E}\left\{\Upsilon_{\text {rch }}\left(T^{*}, T^{*}+\bar{t}\right) \mid \theta\left(T^{*}\right)=i \in \mathcal{S}_{\pi, \text { rec }}\right\}>0$, sendo que a última desigualdade advém do uso de translação do tempo e propriedades de homogeneidade em (4.8). Substituindo estas desigualdades em (4.9) obtemos $\mathcal{E}\left\{\Upsilon_{\mathrm{rch}}(0, \bar{t}+s) \mid \theta(0)=\ell\right\}>0$ para $s, \bar{t}>0$ arbitrários, concluindo a prova.

O fato de que $\pi$-AM e, por exemplo, AE sejam em geral não comparáveis é ilustrado nos exemplos a seguir.

Exemplo 3. Considere o sistema $(A, B, \Lambda)$ com

$$
\begin{gathered}
A_{1}=\left(\begin{array}{ll}
0 & 0 \\
1 & 0
\end{array}\right), \quad A_{2}=\left(\begin{array}{ll}
1 & 0 \\
0 & 0
\end{array}\right), \quad B_{1}=\left(\begin{array}{l}
0 \\
0
\end{array}\right), \quad B_{2}=\left(\begin{array}{l}
1 \\
0
\end{array}\right), \\
\Lambda=\left(\begin{array}{cc}
0 & 0 \\
0.1 & -0.1
\end{array}\right) \quad x(0)=\left(\begin{array}{l}
1 \\
1
\end{array}\right), \quad \pi=\left(\begin{array}{ll}
0 & 1
\end{array}\right) .
\end{gathered}
$$

Note que $\mathcal{S}_{\pi, \mathrm{rec}}=\{1\}$. Calculamos as matrizes de alcançabilidade (4.7) para verificar que posto $\left(\mathbf{R}_{1}\right)=2$ e concluir do Teorema 1 que o sistema é $\pi$-AM. Simulamos a variável $x(t)$ para 1.000 realizações de estados de Markov, e o resultado é ilustrado na Figura 4.1, a qual mostra que $\pi$-AM não significa que $x(t)$ eventualmente alcança o espaço estado inteiro (diferentemente a sistemas lineares sem saltos, vide (Davis, 197r)). Também notamos da Figura 4.1 que se considerarmos $x_{t g t}=\left(\begin{array}{ll}10 & 0\end{array}\right)^{\prime}$ como um "estado alvo", então a bola $\{x: \| x-$ 
$\left.x_{t g t} \|_{\infty}<1 / 2\right\}$ é visitada por $x(t), t \geq 0$, com probabilidade zero, significando que o sistema não é fracamente controlável como definido em (Mariton, 1987). Considerando o problema de alcançabilidade estocástica, como estudado em (Bujorianu e Lygeros, 2007), e o fato de que $\mathcal{P}\left(x(t) \in\left\{x:\left\|x-x_{t g t}\right\|_{\infty}<1 / 2\right\}\right)$, temos então que o sistema não é $A E .^{\mathrm{a}}$

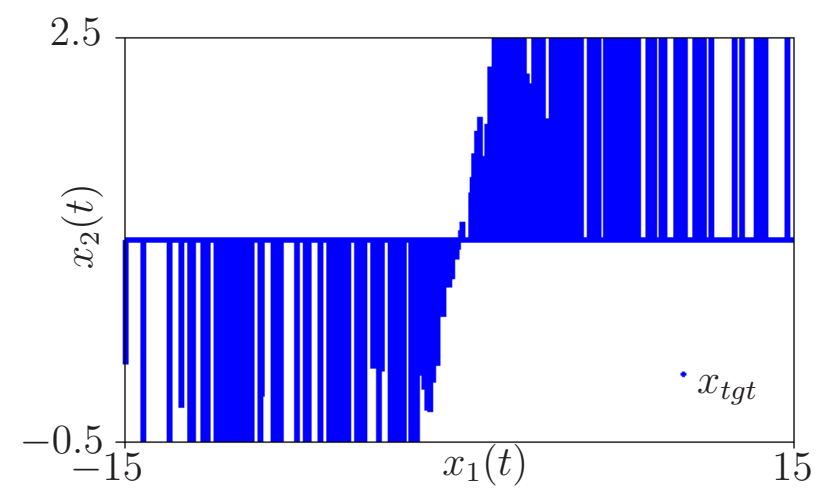

Figura 4.1: Porção de trajetórias correspondentes a 1000 realizações para o processo $\{x(t)\}, t \geq 0$ do sistema no Exemplo 3.

Exemplo 4. Considere o sistema $(A, B, \Lambda)$ com $A_{1}=A_{2}=B_{1}=0, B_{2}=1$, e $\Lambda$ e $\pi$ como no Exemplo 3. Ao implementar o teste do posto do Teorema 1 concluímos que o sistema é não $\pi-A M$, pois $\mathbf{R}_{1}=0$. Mostraremos na sequência que o sistema é $A E$. De fato, desde que o sistema $\left(A_{2}, B_{2}\right)$ é controlável no sentido estândar para sistemas lineares determinísticos, é simples projetar um controle determinístico $w(t), 0 \leq t \leq t_{f}$ resultando em $x\left(t_{f}\right)=x_{t g t}$, devido a que não há saltos neste intervalo de tempo. Assim, $\mathcal{P}\left(\left\|x(t)-x_{t g t}\right\|<\epsilon\right) \geq$ $\mathcal{P}\left(\theta(t)=2,0 \leq t \leq t_{f}\right)=e^{-t_{f}}$.

\subsection{Alcançabilidade Média}

As vezes, a distribuição de probabilidade inicial $\pi$ pode ser desconhecida, o que nos força considerar uma distribuição $\pi$ geral.

Definição 9 (AM). A trinca $(A, B, \Lambda)$ é alcançável em média (AM) se existe $t>0$ tal que $\mathcal{E}\left\{\Upsilon_{\mathrm{rch}}(0, t) \mid \theta(0) \sim \pi\right\}>0$ para qualquer distribuição inicial $\pi$.

\footnotetext{
${ }^{a}$ Assumimos neste trabalho que um sistema é AE se a referida probabilidade é positiva.
} 
Um teste de posto para a AM pode ser obtido em uma maneira bastante direta baseada no teste de $\pi-\mathrm{AM}$ dado no Teorema 1 . A ideia é verificar se o sistema é $\pi^{(\ell)}$-AM para certas distribuições iniciais $\pi^{(\ell)}$, cada uma delas "excitando" um conjunto de estados de estados de Markov recorrentes. Formalmente, sejam os conjuntos irreduzíveis de estados de Markov recorrentes denotados por:

$$
\mathcal{S}_{\text {rec }, 1}, \ldots, \mathcal{S}_{\text {rec }, \bar{m}}
$$

(desta forma a cadeia de Markov está formada por um total de $\bar{m}$ blocos de estados recorrentes mais os estados transientes) e toma as distribuições iniciais associadas:

$$
\pi^{(1)}, \ldots, \pi^{(\bar{m})}
$$

sendo cada $\pi^{(\ell)}$ concentrada em um estado arbitrário pertencendo a $\mathcal{S}_{\text {rec }, \ell}$, isto é, $\pi_{i_{\ell}}^{(\ell)}=\mathcal{P}\left(\theta(0)=i_{\ell}\right)=1$ para algum $i_{\ell} \in \mathcal{S}_{\text {rec }, \ell}$.

Teorema 2. Considere as distribuições iniciais $\pi^{(1)}, \ldots, \pi^{(\bar{m})}$ como definidas acima. As seguintes afirmações são equivalentes:

(i) $(A, B, \Lambda)$ é $A M$.

(ii) $(A, B, \Lambda)$ é $\pi^{(\ell)}-A M$ para todo $\ell=1, \ldots, \bar{m}$.

(iii) $(A, B, \Lambda)$ é $\pi$-AM para toda distribuição de probabilidade $\pi \in \mathbb{R}^{N}$.

Demonstração. Os fatos de que (iii) e (i) sejam equivalentes e que (iii) implica (ii) são triviais. Mostramos $(i i) \Rightarrow(i i i)$ por contradição. Primeiro negamos (iii) assumindo que o sistema é não $\pi$-AM para algum $\pi$, e do Teorema 1 temos

$$
\operatorname{posto}\left(\mathbf{R}_{j^{*}}(A, B, \Lambda, \pi)\right)<n, \text { para algum } j^{*} \in \mathcal{S}_{\pi, \text { rec }}
$$

Agora, assuma (ii) válido e considere $\ell \in\{1, \ldots, \bar{m}\}$ para o qual $j^{*} \in \mathcal{S}_{\text {rec }, \ell}$. O Teorema 1 produz $\operatorname{posto}\left(\mathbf{R}_{j}\left(A, B, \Lambda, \pi^{(\ell)}\right)\right)=n$ para todo $j \in \mathcal{S}_{\pi^{(\ell)} \text {,rec }}$ e em particular para $j=j^{*}$,

$$
\operatorname{posto}\left(\mathbf{R}_{j^{*}}\left(A, B, \Lambda, \pi^{(\ell)}\right)\right)=n
$$

Desde que $j^{*} \in \mathcal{S}_{\text {rec }, \ell}$ temos $\mathcal{S}_{\pi^{(\ell)}, \text { rec }} \subset \mathcal{S}_{\pi, \text { rec }}$, levando em

$$
\operatorname{posto}\left(\mathbf{R}_{j^{*}}\left(A, B, \Lambda, \pi^{(\ell)}\right)\right) \leq \operatorname{posto}\left(\mathbf{R}_{j^{*}}(A, B, \Lambda, \pi)\right) .
$$


Combinando (4.14) e (4.15) obtemos $\operatorname{posto}\left(\mathbf{R}_{j^{*}}(A, B, \Lambda, \pi)\right)=n$, o qual é absurdo em vista de (4.13).

Lema 8. As seguintes afirmações são válidas:

(i) Para uma distribuição de probabilidade inicial dada $\pi$ temos que $(A, B, \Lambda)$ é $\pi-A M$ se e somente se $S_{j}(t)>0$ para todo $t>0$ e $j \in \mathcal{S}_{\pi, \text { rec }}$.

(ii) $(A, B, \Lambda)$ é $A M$ se e somente se para cada distribuição de probabilidade inicial $\pi$ temos que $S_{j}(t)>0$ para todo $t>0$ e $j \in \mathcal{S}_{\pi, \mathrm{rec}}$.

Demonstração. (i). Provamos a necessidade por contradição. Supondo que existe $j \in \mathcal{S}_{\pi \text {,rec }}$ e $\tilde{t}>0$ tais que $S_{j}(\tilde{t})$ não é definida positiva então temos $v^{\prime} S_{j}(\tilde{t}) v=0$ para um certo $v \in \mathbb{R}^{n}$ diferente de zero. Portanto, a equivalência entre $(i)$ e (iii) do Lema 7 produz $v^{\prime} S_{j}(t) v=0$, para todo $t \geq 0$, o qual por sua vez, pela equivalência entre $(i i i)$ e $(i v)$ do Lema 7 leva em $v^{\prime} \mathcal{E}\left\{\Upsilon_{\text {rch }}(0, t) \mid \theta(0)=\right.$ $i\} v=0$ sempre que $i \in \mathcal{S}_{\pi}$ e $j$ sejam estados de Markov comunicantes. Assim, temos que $(A, B, \Lambda)$ é não $\pi$-AM. A prova da suficiência é similar. (ii) Aqui provamos a suficiência por contradição. Suponha que $(A, B, \Lambda)$ é não AM. Então, pelo Teorema 2 existe $\pi^{(\ell)}$ como em (4.12) tal que $(A, B, \Lambda)$ é não $\pi^{(\ell)}$-AM. Então, pela Definição 8 , para todo $t>0$ existe $v \neq 0$ in $\mathbb{R}^{n}$ tal que $v^{\prime} \mathcal{E}\left\{\Upsilon_{\text {rch }}(0, t) \mid \theta(0)=i\right\} v=0$ para algumfor some $i \in \mathcal{S}_{\pi^{(\ell)}}=\mathcal{S}_{\text {rec }, \ell}$. Considere $t>0$ fixo e $j \in \mathcal{S}_{\pi^{(\ell)} \text {,rec }}=\mathcal{S}_{\text {rec }, \ell}$. A equivalência de $(i i i)$ com $(i v)$ do Lema 7 leva em $v^{\prime} S_{j}(t) v=0, t>0$ o qual conclui a prova da suficiência. A necessidade segue de maneira simples usando (iii), (iv) do Lema 7 . 


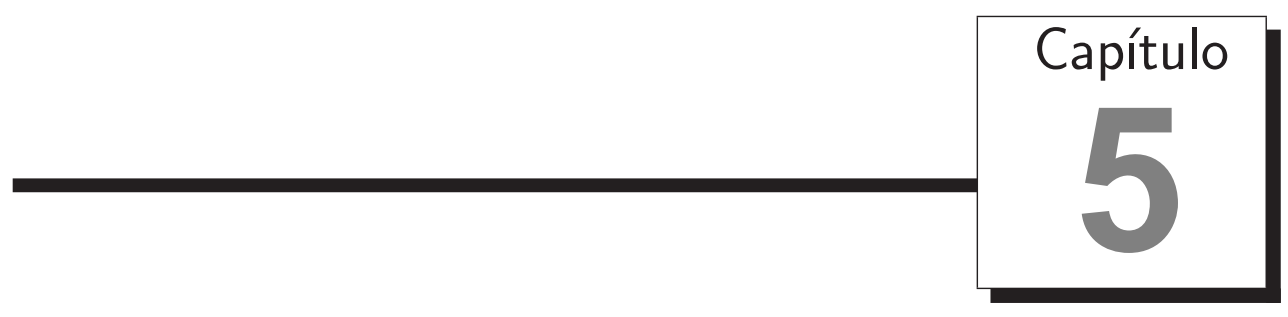

\section{Consequências da alcançabilidade média}

Neste capítulo estudamos aplicações ou usos da AM, considerando o sistema dado por:

$$
\begin{aligned}
& d x(t)=A_{\theta(t)} x(t) d t+B_{\theta(t)} d w(t), \quad x(0)=x_{0}, \quad \theta(0) \sim \pi \\
& d y(t)=C_{\theta(t)} x(t) d t+D_{\theta(t)} d v(t)
\end{aligned}
$$

sendo $\{w(t)\}_{t \geq 0}$ e $\{v(t)\}_{t \geq 0}$ processos de Wiener com operador covariância incremental $I d t$. Durante este capítulo usaremos a notação $(A, B, \Lambda)$ para referir-nos especificamente à equação de estado no sistema em (5.1) e, lembremos que, para o sistema completo usamos a notação $(A, B, C, D, \Lambda)$. Primeiramente, caracterizamos a AM com a positividade das matrizes de segundo momento condicionado, com a interpretação de que existe um "nível mínimo de ruído" no estado, como detalhado na Seção 5.1. Em segundo lugar, no contexto de estimação linear da componente de valor contínuo $x(t)$ do estado, com observação completa da variável de salto, mostramos que AM garante duas propriedades: (i) a matriz de covariância do erro de estimação $Q(t)$ é uma matriz definida positiva (basicamente significando que a estimativa tem um nível mínimo de ruído) e (ii) para estimadores lineares markovianos, a propriedade de que a esperança de $Q(t)$ seja limitada superiormente é invariante a certas perturbações sobre a condição inicial, a qual é algumas vezes referida como estabilidade do filtro. Este resultado de estabilidade do filtro independe da dinâmica do sistema. 


\subsection{Positividade do segundo momento do estado}

Na continuação estendemos a positividade de $S$, mostrada no capítulo anterior, a uma coleção de matrizes denotada por $X(t) \in \mathcal{M}^{n 0}$, a qual está associada ao segundo momento de $x(t)$. Como vimos, $S_{j}$ é definida positiva sempre que $j$ seja um estado de Markov recorrente. Nesta seção obteremos um limitante inferior para $S_{i}(t)$ em um intervalo finito usando o teorema do valor extremo de Weierstrass junto com um procedimento de "reinicializar" $S$ (como na equação (5.6) adiante), assim produzindo $S(t) \geq \gamma I$. Infelizmente, $X$ não é um limitante superior para $S$, o que nos obriga a introduzir um fator de escala $\delta$ para obter $\delta^{-1} X>S(t)$. Isto nos leva a demonstrar que $\mathcal{E}\left\{x(t) x^{\prime}(t)\right\}$ e $X_{i}, i$ recorrente (estas são matrizes componentes da matriz $X$ como veremos a seguir), são limitados inferiormente por matrizes definidas positivas. Seja $X_{j}(t) \in \mathcal{R}^{n 0}$ definida, para cada $j \in \mathcal{S}$, por:

$$
X_{j}(t)=\mathcal{E}\left\{x(t) x(t)^{\prime} 1_{\{\theta(t)=j\}}\right\}, \quad t \geq 0 .
$$

Estas matrizes satisfazem as equações lineares matriciais (veja por exemplo (Fragoso e Costa, 2005)):

$$
\dot{X}_{j}(t)=\mathcal{T}_{A, j}(X(t))+p_{j}(t) B_{j} B_{j}^{\prime}, \quad \text { com } \quad X_{j}(0)=p_{j}(0) \Psi
$$

Observação 3. Uma comparação direta entre (4.1) e (5.3) nos permite concluir que, se $x(0)=0$ e o ruído $w(t)$ é multiplicado por $1 / \sqrt{p_{\theta(t)}(t)}$ sempre que $\theta(t) \in \mathcal{S}_{\pi}$ (de outra forma é multiplicado por zero), então $S$ coincide com $X$. Isto nos dá uma interpretação para $S$ como sendo o segundo momento condicional do sistema quando a amplitude do ruído é convenientemente "modulada" e a condição inicial é estabelecida como zero.

Em vista da Observação 3 , não é uma surpresa que $p_{j}(t)>0$ seja requerido positivo na nossa análise (de forma que $1 / \sqrt{p_{\theta(t)}(t)}$ é bem posto). Uma propriedade bem conhecida para cadeias de Markov a tempo contínuo e espaço estado finito é que dada a continuidade da sua distribuição de probabilidade $p(t)$ existe $\delta>0$ e um intervalo fechado $\left[T_{1}, T_{2}\right] \subset \mathbb{R}, 0<T_{1}<T_{2}$ tal que,

$$
p_{j}(t) \geq \delta \quad j \in \mathcal{S}_{\pi}, t \in\left[T_{1}, T_{2}\right]
$$

(e $\left.p_{j}(t)=0, j \notin \mathcal{S}_{\pi}\right)$, para estados de Markov que podem ser alcançados do 
estado inicial. Para $t \in\left[T_{1}, T_{2}\right]$ definimos $U(t) \in \mathcal{M}^{n 0}$ satisfazendo:

$$
\dot{U}_{j}(t)=\mathcal{T}_{A, j}(U(t))+p_{j}(t) B_{j} B_{j}^{\prime}, \quad \text { com } \quad U_{j}\left(T_{1}\right)=0 .
$$

Também, definimos una variável auxiliar $V(t) \in \mathcal{M}^{n 0}$ para $t \geq T_{1}$ via

$$
\dot{V}_{j}(t)=\mathcal{T}_{A, j}(V(t))+1_{\left\{j \in \mathcal{S}_{\pi}\right\}} B_{j} B_{j}^{\prime}, \quad \text { com } \quad V_{j}\left(T_{1}\right)=0 .
$$

A relação entre $S, V, U$ e $X$ é dada a seguir.

Lema 9. Seja $j \in \mathcal{S}_{\pi, \text { rec. Para } \delta} e\left[T_{1}, T_{2}\right]$ como em (5.4) as seguintes afirmações são válidas:

(i) $V_{j}\left(T_{1}+s\right)=S_{j}(s)$ para todo $s \geq 0$.

(ii) $S e S_{j}(t)>0$ para todo $t>0$, então existe $\gamma>0$ tal que $S_{j}(t) \geq \gamma I$, para todo $t \geq T_{1}$.

(iii) $U_{j}(t) \geq \delta V_{j}(t)$ para todo $t \in\left[T_{1}, T_{2}\right]$.

(iv) $X_{j}(t) \geq U_{j}(t)$ para todo $t \in\left[T_{1}, T_{2}\right]$.

(v) Se para qualquer $\tilde{s}>0$ fixo existe $\gamma>0$ tal que $S_{j}(s) \geq \gamma I$ para todo $s \geq \tilde{s}$, então existe $\rho>0$ tal que $X_{j}(t) \geq \rho I$ para todo $t$ pertencendo a um intervalo $\mathcal{J} \subset\left[T_{1}, T_{2}\right]$.

Demonstração. (i). Imediato de (4.1) e (5.6). (ii). A função definida como $\tilde{S}_{j}=S_{j}-V_{j}$ evolui, para $t \geq T_{1}$, de acordo com:

$$
\dot{\tilde{S}}_{j}(t)=\mathcal{T}_{A, j}(\tilde{S}(t)), \operatorname{com} \quad \tilde{S}_{j}\left(T_{1}\right)=S_{j}\left(T_{1}\right)
$$

De (5.7) e o fato de que $S\left(T_{1}\right) \in \mathcal{M}^{n 0}$ temos que $\tilde{S}_{j}(t) \geq 0$. Isto significa que $S_{j}(t) \geq V_{j}(t)$ para todo $t \geq T_{1}$ e, consequentemente,

$$
S_{j}\left(T_{1}+s\right) \geq S_{j}(s)
$$

para todo $s \geq 0$. Agora, vamos considerar $j \in \mathcal{S}_{\pi \text {,rec }}$ e o conjunto compacto $D_{0}:=\mathbb{S}^{1} \times\left[T_{1}, 2 T_{1}\right]$ sendo $\mathbb{S}^{1}=\left\{v \in \mathbb{R}^{n}:\|v\|=1\right\}$, e definamos para cada $j$ a função $g_{j}: D_{0} \longrightarrow \mathbb{R}^{+}$como $g_{j}(v, s)=v^{\prime} S_{j}(s) v$. $g_{j}$ é contínua e, pelo teorema do valor extremo de Weierstrass, existe $(\bar{v}, \bar{s}) \in D_{0}$ tal que 
$\gamma=\bar{v}^{\prime} S_{j}(\bar{s}) \bar{v}=\min _{(v, s) \in D_{0}} g_{j}(v, s)$. Lembrando que $S_{j}(t)>0$ para todo $t>0$, então temos que $\gamma>0$. Isto produz:

$$
S_{j}(s) \geq \gamma I, \quad s \in\left[T_{1}, 2 T_{1}\right] .
$$

De $(5.8)$ e $(5.9)$ temos que $S_{j}(2 \tilde{t}+\zeta) \geq S_{j}(\tilde{t}+\zeta) \geq \gamma I$, sendo $\zeta$ um número real qualquer tal que $0 \leq \zeta \leq \tilde{t}$. Consequentemente, obtemos $S_{j}(s) \geq \gamma I$ para todo $s \in\left[2 T_{1}, 3 T_{1}\right]$; recursivamente obtemos o resultado. (iii). Considere $V_{j}$ como em (5.6). Para $t \in\left[T_{1}, T_{2}\right]$, definimos $Z(t)=\delta V(t)$, a qual evolui de acordo com:

$$
\dot{Z}_{j}(t)=\mathcal{T}_{A, j}(Z(t))+\delta 1_{\left\{j \in \mathcal{S}_{\pi}\right\}} B_{j} B_{j}^{\prime}, \quad \text { com } \quad Z_{j}\left(T_{1}\right)=0 .
$$

Agora, a função definida como $\tilde{Z}_{j}=U_{j}-Z_{j}$ satisfaz

$$
\dot{\tilde{Z}}_{j}(t)=\mathcal{T}_{A, j}(\tilde{Z}(t))+\left(p_{j}(t)-\delta 1_{\left\{j \in \mathcal{S}_{\pi}\right\}}\right) B_{j} B_{j}^{\prime}, \quad \text { com } \quad \tilde{Z}_{j}\left(T_{1}\right)=0,
$$

sendo $p_{j}(t)-\delta 1_{\left\{j \in \mathcal{S}_{\pi}\right\}} \geq 0$. Então temos que $\tilde{Z}_{j}(t) \geq 0$ para todo $t \in\left[T_{1}, T_{2}\right]$. Portanto $U_{j}(t) \geq \delta V_{j}(t)$ para todo $t \in\left[T_{1}, T_{2}\right]$. (iv). A função definida como $\tilde{U}_{j}=X_{j}-U_{j}$ evolui para $t \in\left[T_{1}, T_{2}\right]$ de acordo com:

$$
\dot{\tilde{U}}_{j}(t)=\mathcal{T}_{A, j}(\tilde{U}(t)), \operatorname{com} \tilde{U}_{j}\left(T_{1}\right)=X_{j}\left(T_{1}\right)
$$

produzindo $\tilde{U}_{j}(t) \geq 0$ para todo $t \in\left[T_{1}, T_{2}\right]$. Assim, obtemos que $X_{j}(t) \geq U_{j}(t)$ para todo $t \in\left[T_{1}, T_{2}\right]$. $(v)$. Seja $T^{*} \in \mathbb{R}$ fixo tal que $0<T^{*}<T_{2}-T_{1}$. Ao estabelecer $\tilde{s}=T^{*}$ temos que existe $\gamma>0$ tal que $S_{j}(s) \geq \gamma I$, para todo $s \geq T^{*}$. Agora, considerando a função $V_{j}$ como em (5.6) e usando $(i i)$ obtemos $V_{j}\left(T_{1}+s\right)=S_{j}(s) \geq \gamma I$ para todo $s \in\left[T^{*}, T_{2}-T_{1}\right]$, notando que $t=T_{1}+s \in \mathcal{J}=\left[T_{1}+T^{*}, T_{2}\right] \subset\left[T_{1}, T_{2}\right]$ sempre que $s \in\left[T^{*}, T_{2}-T_{1}\right]$. Então, ao multiplicar $V$ e $\gamma I$ na última desigualdade por $\delta$ e usando (iii) obtemos $U_{j}(t) \geq \delta V_{j}(t) \geq \rho I$, para todo $t \in \mathcal{J}$ sendo $\rho=\delta \gamma$. Finalmente, usando (iv) concluímos a prova.

Observação 4. Note que, quando consideramos uma distribuição de probabilidade inicial $\pi^{(\ell)}$ como em (4.12) para algum $\ell \in\{1, \ldots, \bar{m}\}$, temos $X_{j}(t)=0$ para todo $t \geq 0$ e $j \notin \mathcal{S}_{\text {rec }, \ell}$. De fato, para um tal $j, p_{j}(t)=0$ para todo $t \geq 0$ $e$ de (5.2) temos $X_{j}(t)=\mathcal{E}\left\{x(t) x(t)^{\prime} \mid \theta(t)=j\right\} p_{j}(t)=0$.

Teorema 3. As seguintes afirmações são equivalentes: 
(i) A trinca $(A, B, \Lambda)$ é $A M$.

(ii) Existe $\rho>0$ tal que para quaisquer condições iniciais $x_{0}$ e $\pi$ temos $X_{j}(t) \geq \rho I$, para todo $t$ que pertence a um intervalo $\mathcal{J} \subset\left[T_{1}, T_{2}\right]$ e todo $j \in \mathcal{S}_{\pi, \text { rec }}$.

(iii) Existe $\rho>0$ tal que para quisquer condições iniciais $x_{0}$ e $\pi$ temos $\mathcal{E}\left\{x(t) x(t)^{\prime}\right\} \geq \rho I$, para todo $t$ que pertence a um intervalo $\mathcal{J} \subset\left[T_{1}, T_{2}\right]$.

Demonstração. $(i) \Rightarrow(i i)$. Suponha que $(A, B, \Lambda)$ é AM. Do Lema 8 (ii) obtemos $S_{j}(t)>0$, para todo $t>0$ e $j \in \mathcal{S}_{\pi, \text { rec }}$, para cada distribuição de probabilidade inicial $\pi$. A afirmação $(i)$ do Lema 9 leva em que existe $\gamma>0$ tal que $S_{j}(s) \geq \gamma I$, para todo $s \geq T_{1}$. Pelo Lema $9(i v)$ temos que existe $\rho>0$ tal que $X_{j}(t) \geq \rho I$ para todo $t \in \mathcal{J} \subset\left[T_{1}, T_{2}\right] .($ ii $) \Rightarrow$ (iii). O fato de que $\mathcal{E}\left\{x(t) x(t)^{\prime}\right\}=\sum_{i \in \mathcal{S}} X_{i}(t) \geq X_{j}(t)$ conclui o resultado. (iii) $\Rightarrow(i)$. Procedemos por contradição, assumindo que $(A, B, \Lambda)$ é não AM. Então, pelo Teorema 2 existe $\pi^{(\ell)}$ como em (4.12) tal que $(A, B, \Lambda)$ é não $\pi^{(\ell)}-\mathrm{AM}$. Lema $8(i)$ implica que $S_{j}(\bar{t})$ é não definida positiva para um certo $\bar{t}>0$ e algum $j \in \mathcal{S}_{\pi^{(\ell)} \text {,rec }}=\mathcal{S}_{\text {rec }, \ell}$, isto é, existe $v \in \mathbb{R}^{n}$ diferente de zero tal que $v^{\prime} S_{j}(\bar{t}) v=0$. Esta igualdade pode ser estendida a $t \geq 0$ geral usando a equivalência de $(i)$ e (ii) do Lema 7 e ao derivar obtemos $v^{\prime} \dot{S}_{j}(t) v=0$ para todo $t \geq 0$. Note que, pela extensão do resultado da descomposição de Cholesky para matrizes semidefinidas positivas temos que para cada $t>0$ existe uma matriz apropriada $Y_{t} \in \mathfrak{R}^{n}$ tal que $S_{j}(t)=Y_{t} Y_{t}^{\prime}$, o qual pode ser empregado para mostrar que $S_{j}(t) v=0$. Desta forma, os termos $v^{\prime}\left(A_{j} S_{j}(t)+S_{j}(t) A_{j}^{\prime}\right) v$ e $\lambda_{j j} v^{\prime} S_{j}(t) v$ são iguais a zero para todo $t \geq 0$ na derivada $v^{\prime} \dot{S}_{j}(t) v$ (veja (4.1)). Portanto temos $\sum_{i \in \mathcal{S}_{\mathrm{rec}, \ell}, i \neq j} v^{\prime} \lambda_{i j} S_{i}(t) v+v^{\prime} B_{j} B_{j}^{\prime} v=0$, o qual é uma soma que envolve matrizes semi-positivas definidas. Em consequência obtemos $v^{\prime} S_{i}(t) v=0$ para

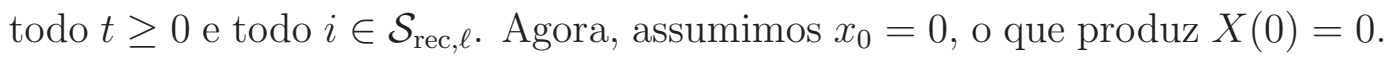
Assim $X(t) \leq S(t)$ o qual implica em $v^{\prime} X_{i}(t) v=0$ para todo $t \geq 0$ e todo $i \in \mathcal{S}_{\text {rec }, \ell}$. Finalmente, obtemos $X_{j}(t)=0$ para todo $t \geq 0$ e $j \notin \mathcal{S}_{\text {rec }, \ell}$ (veja Observação 4) e então $v^{\prime} \mathcal{E}\left\{x(t) x(t)^{\prime}\right\} v=\sum_{i \in \mathcal{S}_{\text {rec }, \ell}} v^{\prime} X_{i}(t) v=0$ para todo $t \geq 0$.

A razão simples pela qual não podemos tomar $T_{2}$ tendendo pro infinito nos resultados prévios é que (5.4) é válida somente para $T_{2}$. Porém, se restringirmos a análise à situação em que só estados de Markov recorrentes são visitados, podemos substituir $\mathcal{S}_{\pi}$ por $\mathcal{S}_{\pi, \text { rec }}$ em (5.4) (e em outras passagens relevantes incluindo(4.1)) a qual é válida agora para todo $t \geq T_{1}$. No caso particular 
em que for desejado somente positividade (não uniforme) da esperança do segundo momento, poderíamos estabelecer $T_{1}=0$. Juntando estes argumentos, obtemos o seguinte resultado. Seja $\Pi$ o conjunto de distribuições que "excitam somente estados de Markov recorrentes"; formalmente, se estabelecermos $\mathcal{S}_{\text {rec }}=\bigcup_{\ell=1}^{m} \mathcal{S}_{\text {rec }, \ell}$, com $\mathcal{S}_{\text {rec }, \ell}$ como em (4.11), então:

$$
\Pi=\left\{\pi \in \mathbb{R}^{N}: \sum_{i \in \mathcal{S}_{\text {rec }}} \pi_{i}=1\right\}
$$

Definição 10. O sistema $(A, B, \Lambda)$ é $\Pi-A M$ se e somente se este é $\pi$-AM para todo $\pi \in \Pi$.

Corolário 5. As seguintes afirmações são equivalentes:

(i) A trinca $(A, B, \Lambda)$ é $\Pi-A M$.

(ii) Existem $\rho>0$ e $T>T_{1}$ tais que para quaisquer condições iniciais $x_{0} e$ $\pi \in \Pi$ temos $X_{j}(t) \geq \rho I$ para todo $t \geq T$ e todo $j \in \mathcal{S}_{\pi, \text { rec }}$.

(iii) Existem $\rho>0$ e $T>T_{1}$ tais que para quaisquer condições iniciais $x_{0} e$ $\pi \in \Pi$ temos $\mathcal{E}\left\{x(t) x(t)^{\prime}\right\} \geq \rho I$ para todo $t \geq T$.

(iv) Para quaisquer condições $x_{0} e \pi \in \Pi$ temos $X_{j}(t)>0$ para todo $t>0 e$ todo $j \in \mathcal{S}_{\pi, \text { rec }}$.

Exemplo 5 (AM e a estrutura da cadeia de Markov). Considere o sistema $(A, B, \Lambda)$ com

$$
\begin{gathered}
A_{1}=\left(\begin{array}{cc}
-1 & 0.05 \\
10 & -1
\end{array}\right), A_{2}=\left(\begin{array}{cc}
1 & -0.9 \\
1.1 & 0.6
\end{array}\right), A_{3}=\left(\begin{array}{cc}
0 & -1.7 \\
1.4 & -0.5
\end{array}\right), \\
B_{1}=\left(\begin{array}{ccc}
1 & 0.2 & -1.9 \\
-0.1 & 1.4 & -0.3
\end{array}\right), \quad B_{2}=B_{3}=0,
\end{gathered}
$$

x(0) com distribuição Gaussiana com média zero e matriz covariância $100 I$. Deveremos considerar valores diferentes para $\pi$ e $\Lambda$. Sejam $\pi_{a}=\pi_{c}=\left(\begin{array}{lll}0 & 0 & 1\end{array}\right)$, $\pi_{b}=\left(\begin{array}{lll}0 & 1 & 0\end{array}\right)$,

$$
\Lambda_{a}=\left(\begin{array}{ccc}
-1 & 0.5 & 0.5 \\
0.5 & -2 & 1.5 \\
0.9 & 0.1 & -1
\end{array}\right), \quad \Lambda_{b}=\left(\begin{array}{ccc}
-1 & 1 & 0 \\
2 & -2 & 0 \\
0.9 & 0.1 & -1
\end{array}\right), \quad \Lambda_{c}=\left(\begin{array}{ccc}
-1 & 1 & 0 \\
2 & -2 & 0 \\
0 & 0 & 0
\end{array}\right)
$$



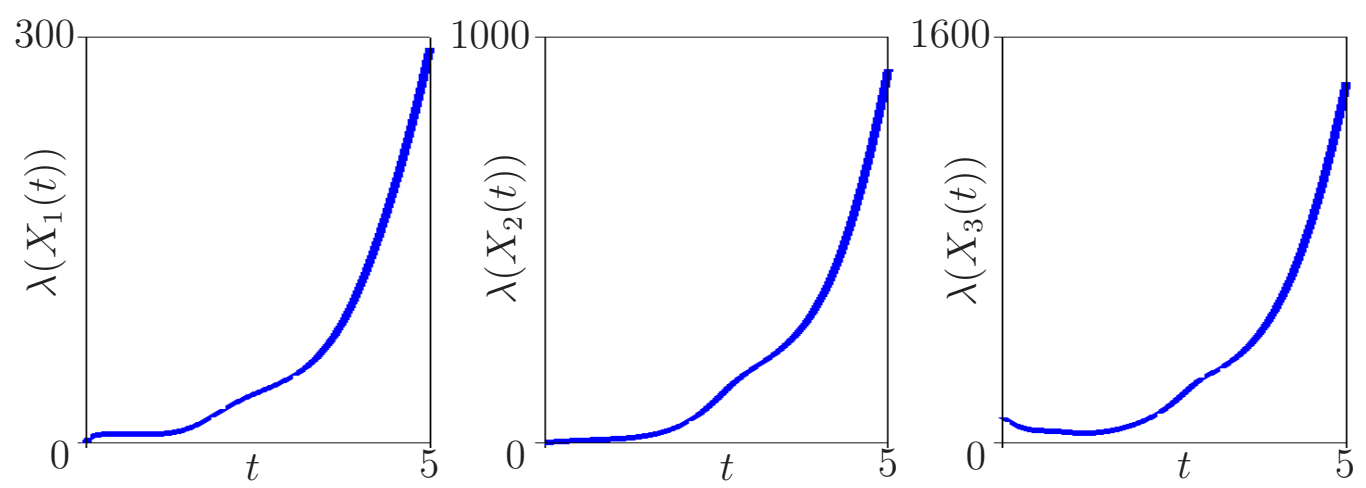

Figura 5.1: Menor autovalor de $X_{j}(t), j \in \mathcal{S}=\{1,2,3\}$, para o sistema no Exemplo 5(a).

(a) Sejam $\pi=\pi_{a}$ e $\Lambda=\Lambda_{a}$. A cadeia de Markov é ergódica de forma que dispomos só de um conjunto irredutivel $(\bar{m}=1)$, dado por $\mathcal{S}_{\mathrm{rec}, 1}=\{1,2,3\}$. Tomamos por exemplo $\pi^{(1)}=\left(\begin{array}{lll}1 & 0 & 0\end{array}\right)$, calculamos as matrizes de alcançabilidade usando (4.7) e vemos que posto $\left(\mathbf{R}_{j}\right)=2$, para $j \in \mathcal{S}=\{1,2,3\}$. Do Teorema 2 concluímos que os sistemas são $\pi^{(1)}-A M$ e AM. Note da Figura 5.1 que $X_{j}(t)$ é definida positiva para $t>0$ e $j \in \mathcal{S}$ e consequentemente $\mathcal{E}\left\{x(t) x(t)^{\prime}\right\}>0$ confirmando o Teorema 3. AM implica П-AM, e a Figura 5.1 confirma também o Corolário 5 (obtivemos $\rho \approx 45$ and $T=2.5$ ).

(b) Considere $\pi=\pi_{b}$ e $\Lambda=\Lambda_{b}$. Temos $\bar{m}=1, \mathcal{S}_{\text {rec }, 1}=\{1,2\}$. Para $\pi^{(1)}=\left[\begin{array}{lll}1 & 0 & 0\end{array}\right]$ calculamos as matrizes de alcançabilidade por (4.7) e concluímos que $(A, B, \Lambda)$ é $\pi^{(1)}-A M$ e AM. Obtemos que $X_{3}(t)=0$ e $X_{j}$ é positiva definida somente para estados de Markov recorrentes (como visto na Figura 5.2), confirmando Teorema 3 e Corolário 5.

(c) Sejam $\pi=\pi_{c}$ e $\Lambda=\Lambda_{c}$. Neste caso temos $\bar{m}=2, \mathcal{S}_{\text {rec }, 1}=\{1,2\}$ e $\mathcal{S}_{\text {rec }, 2}=\{3\}$; podemos estabelecer $\pi^{(1)}=\left[\begin{array}{lll}1 & 0 & 0\end{array}\right]$ e $\pi^{(2)}=\left[\begin{array}{lll}0 & 0 & 1\end{array}\right]$ e usamos (4.7) para obter $\operatorname{posto}\left(\mathbf{R}_{j}\right)=2$, para $j \in \mathcal{S}_{\pi^{(1)} \text {,rec }}=\mathcal{S}_{\text {rec }, 1}$, e posto $\left(\mathbf{R}_{3}\right)=0$, assim concluindo que o sistema é $\pi^{(1)}-A M$ e não $\pi^{(2)}-A M$. Consequentemente, $(A, B, \Lambda)$ é não $A M$. Note também que $\mathcal{E}\left\{x(t) x(t)^{\prime}\right\}$ tende a zero quando $t$ cresce como ilustrado na Figura 5.3. Em este exemplo, $\pi \notin \Pi$ portanto Corolário 5 não aplica. 

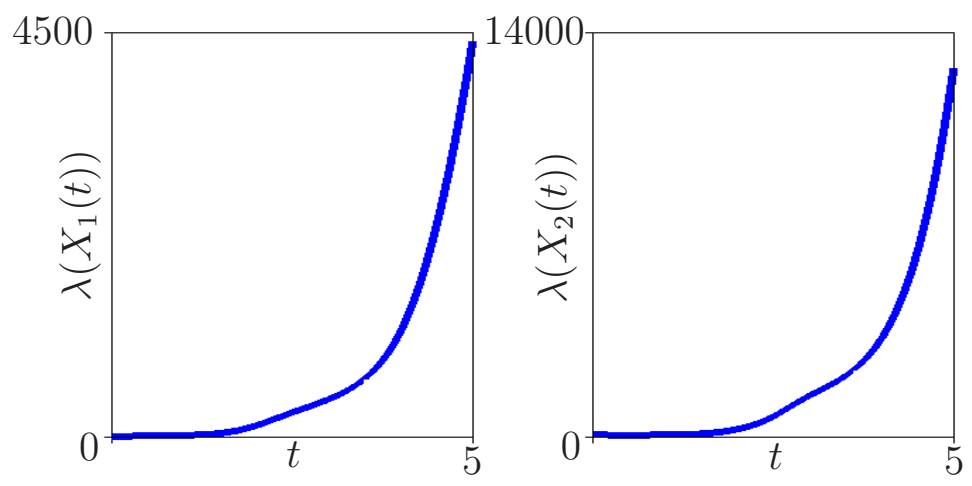

Figura 5.2: Menor autovalor de $X_{j}(t), j \in\{1,2\}$ para o sistema no Exemplo $5(b)$.

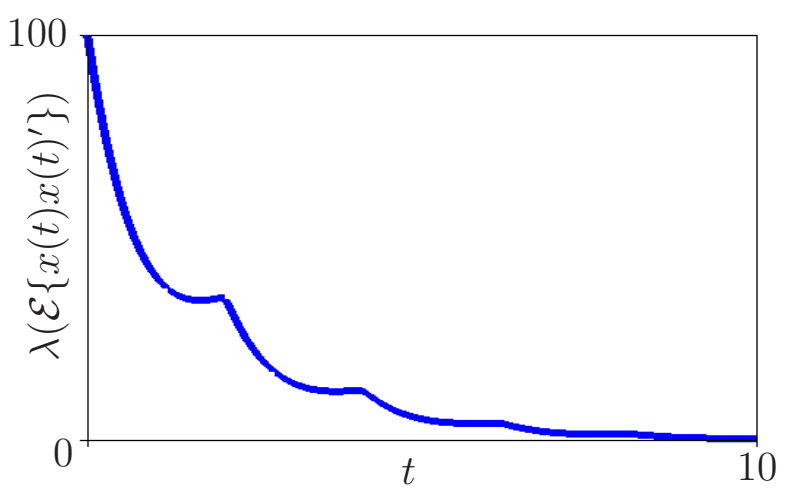

Figura 5.3: Menor autovalor de $\mathcal{E}\left\{x(t) x(t)^{\prime}\right\}, t \in[0,5]$, para o sistema no Exemplo 5(c).

\subsection{Propriedades da covariância do erro de estimação de SLSM}

Em esta seção estudamos estimadores lineares para SLSM a tempo contínuo. É sabido que, o melhor estimador linear para esta classe de sistemas é o filtro de Kalman-Bucy (FKB) aparecendo por exemplo em (Ji e Chizeck, 1992); porém, a implementação do FKB envolve resolver uma equação matricial diferencial parametrizada por $\theta$, tornando muito mais complexo pre-calcular a sua solução e o ganho de Kalman. Motivado por esta limitação do FKB surge então o ELQMM proposto em (Costa et al., 2013) como filtro alternativo não só linear mas também markoviano, com a principal vantagem (em relação ao FKB) de que seus ganhos e matrizes de covariância do erro de estimação po- 
dem ser pre-computadas, fazendo com que a estimativa seja muito mais fácil de ser calculada em aplicações reais. O ELQMM tem algumas características importantes: ele é ótimo em um certo sentido e satisfaz um princípio de separação quando usado em problemas de controle. No Apêndice B é apresentado um estudo de caso comparativo entre o FKB e o ELQMM.

\section{Estimadores lineares e positividade da covariância do erro}

Vamos estudar propriedades de estimadores lineares de SLSM tais como a positividade da covariância do erro de estimação. Um estimador linear (veja (Kwakernaak e Sivan, 1972)) é dado como:

$$
\begin{aligned}
d \hat{x}_{K}(t) & =A_{\theta(t)}(t) \hat{x}_{K}(t) d t+K_{t}\left(d y(t)-C_{\theta(t)}(t) \hat{x}_{K}(t) d t\right), \\
\hat{x}_{K}(0) & =\hat{x}_{K}
\end{aligned}
$$

sendo $\hat{x}_{K}$ um certo vetor em $\mathbb{R}^{n}$ e o processo $K=\left\{K_{t}\right\}_{t \geq 0}$ pertencendo ao conjunto de ganhos $\mathfrak{K}=\left\{K: \mathbb{R}_{0}^{+} \longrightarrow \mathcal{R}^{n, r}\right\}$. De fato, os elementos em $\mathfrak{K}$ poderiam realmente ser funções com valores $K(t, \theta(s), 0 \leq s \leq t)$. Para uma função arbitrária $K \in \mathfrak{K}$, ao estabelecer $\tilde{x}(t)=x(t)-\hat{x}_{K}(t)$, é sabido que $\tilde{x}_{K}(t)$ satisfaz a equação:

$$
d \tilde{x}_{K}(t)=\hat{A}(t) \tilde{x}_{K}(t) d t+\hat{B}(t) d \nu(t)
$$

sendo as matrizes $\hat{A}(t), \hat{B}(t)$ e o vetor $d \nu(t)$ definidos como $\hat{A}(t)=A_{\theta(t)}-$ $K_{t} C_{\theta(t)}, \hat{B}(t)=\left[\begin{array}{ll}B_{\theta(t)} & -K_{t} D_{\theta(t)}\end{array}\right]$, e $d \nu(t)=\left[\begin{array}{ll}d w(t) & d v(t)\end{array}\right]^{\prime}$ respectivamente. Definimos $Q_{K}(t)=\mathcal{E}\left\{\tilde{x}_{K}(t) \tilde{x}_{K}(t)^{\prime} \mid \mathcal{G}_{t}\right\}$, sendo $\mathcal{G}_{t}=\sigma\{\theta(s): 0 \leq s \leq t\}$ a $\sigma$-álgebra gerada pela cadeia de Markov até o instante $t$. Usando ferramentas usuais da teoria de filtragem para sistemas lineares variantes no tempo, podemos derivar a seguinte equação diferencial linear em termos de $Q_{K}$ :

$$
\begin{aligned}
\dot{Q}_{K}(t)=( & \left.A_{\theta(t)}-K_{t} C_{\theta(t)}\right) Q_{K}(t)+Q_{K}(t)\left(A_{\theta(t)}-K_{t} C_{\theta(t)}\right)^{\prime}+B_{\theta(t)} B_{\theta(t)}^{\prime} \\
& +K_{t} D_{\theta(t)} D_{\theta(t)}^{\prime} K_{t}^{\prime}
\end{aligned}
$$

Proposição 11. Suponha que $(A, B, \Lambda)$ é AM. Então, dada qualquer $K \in \mathfrak{K}$ temos que $\mathcal{E}\left\{Q_{K}(t)\right\}>0$ para todo $t>0$, ou, equivalentemente, não existe operador projeção não trivial $\mathfrak{P}$ tal que $\mathfrak{P}\left(\tilde{x}_{K}(t)\right)=0$ (q.c.).

Demonstração. Procedemos por contradição supondo que existe $t>0$ e $v \neq 0$ tais que $v \mathcal{E}\left\{Q_{K}(t)\right\} v=0$, isto é, $v^{\prime} Q_{K}(t) v=0$ (q.c.). Considere $\Gamma(\cdot, \cdot)$ a 
matriz de transição de estados do sistema $\dot{z}(t)=\left(A_{\theta(t)}-K_{t} C_{\theta(t)}\right) z(t)$. É possível verificar que $\frac{d}{d \tau}\left(\Gamma(t, \tau) Q_{K}(\tau) \Gamma(t, \tau)^{\prime}\right) \geq 0$ (q.c.) para todo $\tau \in[0, t]$ (veja (Anderson, 1971, Lema 3.1)). Assim, $\frac{d}{d \tau}\left(v^{\prime} \Gamma(t, \tau) Q_{K}(\tau) \Gamma(t, \tau)^{\prime} v\right) \geq 0$, $\tau \in[0, t]$, o qual implica que a função $v^{\prime} \Gamma(t, \tau) Q_{K}(\tau) \Gamma(t, \tau)^{\prime} v$ seja crescente em $\tau \in[0, t]$ (q.c.); ao combinar isto $\operatorname{com} v^{\prime} Q_{K}(t) v=0$ (q.c.), temos:

$$
v^{\prime} \Gamma(t, \tau) Q_{K}(\tau) \Gamma(t, \tau)^{\prime} v=0 \text {, (q.c.), para todo } \tau \in[0, t] .
$$

Isto também implica que $\frac{d}{d \tau}\left(v^{\prime} \Gamma(t, \tau) Q_{K}(\tau) \Gamma(t, \tau)^{\prime} v\right)=0$ (q.c.) para todo $\tau \in[0, t]$ o qual produz:

$$
B_{\theta(\tau)}^{\prime} \Gamma(t, \tau)^{\prime} v=0 \quad \text { e } \quad K_{\tau}^{\prime} \Gamma(t, \tau)^{\prime} v=0 \text {, (q.c.) para } 0 \leq \tau \leq t
$$

Notando que

$$
\frac{d \Gamma(t, \tau)}{d \tau}=-\Gamma(t, \tau)\left(A_{\theta(\tau)}-K_{\tau} C_{\theta(\tau)}\right)
$$

e usando (5.18) obtemos:

$$
v^{\prime} \frac{d \Gamma(t, \tau)}{d \tau}=-v^{\prime} \Gamma(t, \tau) A_{\theta(\tau)}, \text { (q.c.) para } 0 \leq \tau \leq t . .
$$

Consideremos agora a matriz de transição de estado $\Phi$ aparecendo na solução de (2.8). É simples verificar para cada realização da cadeia que:

$$
v^{\prime} \frac{d \Phi(t, \tau)}{d \tau}=-v^{\prime} \Phi(t, \tau) A_{\theta(\tau)}, \text { para } 0 \leq \tau \leq t
$$

De (5.19) e (5.20), e o fato de que $\Gamma(t, t)=\Phi(t, t)=I$

$$
\Gamma(t, \tau)^{\prime} v=\Phi(t, \tau)^{\prime} v, \text { (q.c.) para } 0 \leq \tau \leq t
$$

Na sequência mostraremos que $(A, B, \Lambda)$ é não $\mathrm{AM}$, considerando uma versão do sistema (2.8) com estatísticas diferentes da condição inicial; com o fim de evitar qualquer confusão com (2.8), vamos introduzir o sistema

$$
d \zeta(t)=A_{\theta(t)} \zeta(t) d t+B_{\theta(t)} d w(t)
$$

cuja condição inicial satisfaz $\mathcal{E}\{\zeta(0)\}=0$ e $\mathcal{E}\left\{\zeta(0) \zeta(0)^{\prime}\right\}=Q_{K}(0)$. Assumindo a hipótese de $\mathrm{AM}$ da trinca $(A, B, \Lambda)$, notamos que (5.22) é AM porque a noção de AM é indiferente das estatísticas da condição inicial. Também introduzimos 
o processo $\mathcal{X}(t)=\mathcal{E}\left\{\zeta(t) \zeta(t)^{\prime} \mid \mathcal{G}_{t}\right\}$, o qual satisfaz:

$$
\mathcal{X}(s)=\Phi(s, 0) Q_{K}(0) \Phi(s, 0)^{\prime}+\int_{0}^{s} \Phi(s, \tau) B_{\theta(\tau)} B_{\theta(\tau)}^{\prime} \Phi(s, \tau)^{\prime} d \tau
$$

para todo $s>0$ e cada realização da cadeia de Markov. Então, (5.17) com $\tau=0$, a primeira igualdade em (5.18) e (5.21) produzem $v^{\prime} \mathcal{X}(t) v=0$ (q.c.). Portanto temos $v^{\prime} \mathcal{E}\{\mathcal{X}(t)\} v=v^{\prime} \mathcal{E}\left\{\zeta(t) \zeta(t)^{\prime}\right\} v=0$ o qual é uma contradição com a AM do sistema (5.22) (veja Corolário 5, (iii)). Assim, mostramos que $\mathcal{E}\left\{Q_{K}(t)\right\}>0$ para todo $t>0$. Só resta mostrar que para qualquer operador projeção não trivial $\mathfrak{P}$ atuando sobre subespaços de $\mathbb{R}^{n}$ obtemos $\mathfrak{P} \tilde{x}_{K}(t) \neq 0$ para todo $t>0$. De fato, vamos supor que existe $\mathfrak{P}$ tal que $\mathfrak{P} \tilde{x}_{K}(t)=0$ (q.c.) para algum $t>0$. Isto é, existe $v \in \mathbb{R}^{n}, v \neq 0$, tal que $\left(v^{\prime} \tilde{x}_{K}(t)\right) v=v v^{\prime} \tilde{x}_{K}(t)=$ 0 (q.c.) para algum $t>0$ e $v \neq 0$, o qual é equivalente a $v^{\prime} \tilde{x}_{K}(t)=0$ (q.c.). Isto é equivalente a $v^{\prime} \mathcal{E}\left\{Q_{K}(t)\right\} v=0$ para algum $t>0$ e $v \neq 0$, o qual é um absurdo em vista da positividade de $\mathcal{E}\left\{Q_{K}(t)\right\}$ para todo $t>0$.

\section{Estabilidade de estimadores lineares markovianos}

Consideraremos nesta seção estimadores lineares cujos ganhos pertencem a um conjunto $\mathfrak{K}_{M} \subset \mathfrak{K}$ de processos markovianos de valor matricial, isto é, todo processo $\left\{K_{t}\right\}_{t \geq 0} \in \mathfrak{K}_{M}$ é tal que $K_{t}=K_{\theta(t)}(t)$ para cada $t \geq 0$. Chamamos estes estimadores de lineares markovianos e são dados como:

$$
\begin{aligned}
d \hat{x}(t) & =A_{\theta(t)}(t) \hat{x}(t) d t+K_{\theta(t)}(t)\left(d y(t)-C_{\theta(t)}(t) \hat{x}(t) d t\right), \\
\hat{x}(0) & =\hat{x}
\end{aligned}
$$

com $\hat{x} \in \mathbb{R}^{n}$. Estabelecendo $\tilde{x}(t)=x(t)-\hat{x}(t)$, pode ser mostrado que:

$$
d \tilde{x}(t)=\left(A_{\theta(t)}-K_{\theta(t)}(t) C_{\theta(t)}\right) \tilde{x}(t) d t+B_{\theta(t)} d w(t)-K_{\theta(t)}(t) D_{\theta(t)} d v(t),
$$

sendo $K(t)=\left(K_{1}(t), \ldots, K_{N}(t)\right)$ a coleção de ganhos de dimensões apropriadas. Como antes, definimos $Q(t)=\mathcal{E}\left\{\tilde{x}(t) \tilde{x}(t)^{\prime} \mid \mathcal{G}_{t}\right\}$, da qual podemos obter a seguinte equação diferencial:

$$
\begin{gathered}
\dot{Q}(t)=\left(A_{\theta(t)}-K_{\theta(t)}(t) C_{\theta(t)}\right) Q(t)+Q(t)\left(A_{\theta(t)}-K_{\theta(t)}(t) C_{\theta(t)}\right)^{\prime}+B_{\theta(t)} B_{\theta(t)}^{\prime} \\
+K_{\theta(t)}(t) D_{\theta(t)} D_{\theta(t)}^{\prime} K_{\theta(t)}^{\prime}(t), \quad t \geq 0 .
\end{gathered}
$$


Também introduzimos a matriz em média

$$
Q_{j}(t)=\mathcal{E}\left\{Q(t) 1_{\{\theta(t)=j\}}\right\}, \quad j \in \mathcal{S} .
$$

Derivando a matriz $Q_{j}(t)$ obtemos:

$$
\begin{aligned}
d Q_{j}(t)= & \mathcal{E}\left\{\left[\left(A_{\theta(t)}-K_{\theta(t)}(t) C_{\theta(t)}\right) Q(t)+Q(t)\left(A_{\theta(t)}-K_{\theta(t)}(t) C_{\theta(t)}\right)^{\prime}\right.\right. \\
& \left.\left.+B_{\theta(t)} B_{\theta(t)}^{\prime}+K_{\theta(t)}(t) D_{\theta(t)} D_{\theta(t)}^{\prime} K_{\theta(t)}(t)^{\prime}\right] d t 1_{\{\theta(t)=j\}}+Q(t) d\left(1_{\{\theta(t)=j\}}\right)\right\} .
\end{aligned}
$$

Assim, calculando a esperança em (5.27) e usando (5.26), obtemos:

$$
\begin{aligned}
d Q_{j}(t)= & {\left[\left(A_{j}-K_{j}(t) C_{j}\right) Q_{j}(t)+Q_{j}(t)\left(A_{j}-K_{j}(t) C_{j}\right)^{\prime}+\left(B_{j} B_{j}^{\prime}\right.\right.} \\
& \left.\left.+K_{j} D_{j} D_{j}^{\prime} K_{j}^{\prime}\right) p_{j}(t)\right] d t+\mathcal{E}\left\{Q(t) 1_{\{\theta(t+d t)=j\}}\right\}-Q_{j}(t) .
\end{aligned}
$$

Agora, usando algumas propriedades da esperança junto com a as taxas de probabilidades transição e algumas manipulações algébricas, calculamos:

$$
\begin{aligned}
\mathcal{E}\left\{Q(t) 1_{\{\theta(t+d t)=j\}}\right\} & =\sum_{i=1}^{N} \mathcal{E}\left\{Q(t) 1_{\{\theta(t+d t)=j\}} 1_{\{\theta(t)=i\}}\right\} \\
& =\sum_{i=1}^{N} \mathcal{E}\left\{\mathcal{E}\left\{Q(t) 1_{\{\theta(t+d t)=j\}} 1_{\{\theta(t)=i\}} \mid \mathcal{G}_{t}\right\}\right\} \\
& =\sum_{i=1}^{N} Q_{i}(t) \mathcal{P}(\theta(t+d t)=j \mid \theta(t)=i) \\
& =\sum_{\substack{i=1 \\
i \neq j}}^{N}\left(\lambda_{i j} d t+o(d t)\right) Q_{i}(t)+\left(1+\lambda_{j j} d t+o(d t)\right) Q_{j}(t) .
\end{aligned}
$$

Portanto, de (5.28) e (5.29) obtemos:

$$
\begin{aligned}
\dot{Q}_{j}(t)=( & \left.A_{j}-K_{j}(t) C_{j}\right) Q_{j}(t)+Q_{j}(t)\left(A_{j}-K_{j}(t) C_{j}\right)^{\prime}+\sum_{i=1}^{N} \lambda_{i j} Q_{i}(t) \\
& +\left(B_{j} B_{j}^{\prime}+K_{j}(t) D_{j} D_{j}^{\prime} K_{j}^{\prime}(t)\right) p_{j}(t) .
\end{aligned}
$$

Na continuação apresentamos um resultado de positividade da covariância do erro $Q_{j}(t), j \in \mathcal{S}$, análogo ao da Proposição 11. Para tal fim é importante considerar a validade da seguinte.

Hipótese 1. $\{\theta(t)\}_{t \geq 0}$ é ergódica e $p_{j}(t)$ é positiva para todo $j \in \mathcal{S}$ e $t \geq 0$. 
Proposição 12. Se $(A, B, \Lambda)$ é $A M$ então $Q_{j}(t)>0$ para todo $t>0$ e $j \in$ $\mathcal{S}$, ou, equivalentemente, não existe operador projeção não trivial $\mathfrak{P}$ tal que $\mathfrak{P}(\tilde{x}(t))=0$ (q.c.).

Demonstração. Em esta prova, por brevidade escrevemos $E$ (q.c. $t, j$ ) para qualquer evento $E$ tal que $\mathcal{P}(E \mid \theta(t)=j)=1$. Procedemos por contradição supondo que existe $t>0$ e $v \neq 0$ tais que $v Q_{j}(t) v=0$, para algum $j \in \mathcal{S}$. Lembrando que assumimos $p_{j}(t)>0$, o anterior é equivalente a $v^{\prime} \mathcal{E}\{Q(t) \mid \theta(t)=j\} v=0$, produzindo $\mathcal{P}\left(v^{\prime} Q(t) v=0 \mid \theta(t)=j\right)=1$, isto é, $v^{\prime} Q(t) v=0$ (q.c. $t, j$ ). Se $\tilde{\Gamma}(\cdot, \cdot)$ é a matriz de transição de estados de $z=$ $\left(A_{\theta(s)}-K_{\theta(s)}(s) C_{\theta(s)}\right) z$ temos que $\frac{d}{d \tau}\left(\tilde{\Gamma}(t, \tau) Q(\tau) \Gamma(t, \tau)^{\prime}\right) \geq 0$ (q.c.) para todo $\tau \in[0, t]$ como mostrado na prova da Proposição 11. De fato, procedendo em diante como na prova da Proposição 11 considerando $Q(t)$ e $\tilde{\Gamma}(\cdot, \cdot)$ ao invés de $Q_{K}(t)$ e $\Gamma(\cdot, \cdot)$ respectivamente, chegamos em $v^{\prime} \mathcal{X}(t) v=0$ (q.c. $\left.t, j\right)$. Considerando o processo $\zeta(t)$ em $(5.22) \operatorname{com} \mathcal{E}\{\zeta(0)\}=0$ e $\mathcal{E}\left\{\zeta(0) \zeta(0)^{\prime}\right\}=Q(0)$, temos $0=v^{\prime} \mathcal{E}\{\mathcal{X}(t) \mid \theta(t)=j\} v=v^{\prime} \mathcal{E}\left\{\zeta(t) \zeta(t)^{\prime} \mid \theta(t)=j\right\} v=v^{\prime} \mathcal{E}\left\{\zeta(t) \zeta(t)^{\prime} 1_{\{\theta(t)=j\}}\right\} v$ o qual é uma contradição com a AM do sistema (5.22) (veja Corolário 5, (iv)). Assim, mostramos que

$$
Q_{j}(t)>0 \text { para todo } t>0, j \in \mathcal{S} \text {. }
$$

$\mathfrak{P} \tilde{x}(t) \neq 0$ para todo $t>0$ segue de maneira similar a como foi feito na prova da Proposição 11.

Estudaremos agora propriedades tais como a estabilidade do filtro em (5.23) sujeita a "erros" nas estatísticas das condições iniciais do sistema. No caso por exemplo do ELQMM, Fragoso e Costa (2010) mostra que $Q_{j}(t)$ converge quando $t$ tende a infinito sempre que o sistema seja detetável na média quadrática, indiferente da $\mathrm{AM}$ de $(A, B, \Lambda)$. No restante desta seção assumimos limitação de $Q_{j}, j \in \mathcal{S}$.

Hipótese 2. Existe uma matriz $Q^{*} \in \mathfrak{R}^{n 0}$ tal que $Q_{j}(t) \leq Q^{*}$ para todo $t \geq 0$ $e j \in \mathcal{S}$.

Esta é uma hipótese de trabalho para maior simplicidade dos resultados mas têm condições suficientes para tal limitação na literatura, do tipo Sdetetabilidade/MS-detetabilidade, que podem ser verificadas facilmente. Expandindo sobre a limitação de $Q$, estudamos a invariância desta propriedade sob perturbações nas estatísticas iniciais do modelo. Este assunto foi abordado na literatura de filtragem, com algumas variações, sob a denominação 
geral de estabilidade do filtro e limitação do erro do filtro ou sensibilidade aos erros para o caso do filtro de Kalman-Bucy, veja por exemplo (Jazwinski, 1970; Nishimura, 1967), para sistemas sem saltos de Markov. Para SLSM a tempo discreto alguns estudos preliminares foram apresentados em (Gomes e Costa, 2010). Aqui, introduzimos perturbações na condição inicial de (5.25) e estudamos sua nova solução, denotada por $P(t)$, a qual satisfaz:

$$
\begin{aligned}
\dot{P}(t, \Sigma)= & \left(A_{\theta(t)}-K_{\theta(t)}(t) C_{\theta(t)}\right) P(t, \Sigma)+P(t, \Sigma)\left(A_{\theta(t)}-K_{\theta(t)}(t) C_{\theta(t)}\right)^{\prime} \\
& +B_{\theta(t)} B_{\theta(t)}^{\prime}+K_{\theta(t)}(t) D_{\theta(t)} D_{\theta(t)}^{\prime} K_{\theta(t)}(t)^{\prime} \\
P(0, \Sigma)=\Sigma &
\end{aligned}
$$

sendo $\Sigma \in \mathfrak{R}^{n 0}$. Se $P$ é limitada em média para qualquer $\Sigma \in \mathfrak{R}^{n 0}$, dizemos que o filtro é estável. A estabilidade é relevante quando implementamos o filtro já que a condição inicial modelada $Q(0)$ poderia conter erros, e mesmo erros infinitesimais poderiam causar divergência rápida de $P$, veja Exemplo 7 . $Q(t)$ pode ser interpretada como a "covariância do erro modelada" ligada com o sistema $(A, B, \Lambda)$, sendo que corresponde ao erro de estimação em relação a um sistema modelo, enquanto que $P(t)$ é interpretada como a "covariância do erro real" das estimativas obtidas com esta função ganho.

Definição 11. Dizemos que o estimador linear markoviano em (5.23) é estável se, para cada $\Sigma \in \mathfrak{R}^{n 0}$, existe $P^{*} \geq 0$ tal que $\mathcal{E}\{P(t)\} \leq P^{*}$ para todo $t \geq 0$, sendo $P(t)$ dado por (5.32).

Teorema 4. Se a Hipótese 2 é satisfeita e $Q_{j}(t)>0$ para todo $t>0$ e $j \in \mathcal{S}$, então o filtro linear markoviano em (5.23) é estável.

Demonstração. Dado que $Q_{j}(t)>0$ para todo $t>0$ e $j \in \mathcal{S}$, temos que para um $t^{*}>0$ fixo temos $Q_{j}\left(t^{*}\right) \geq \mu_{j} I$, para algum $\mu_{j}>0, j \in \mathcal{S}$. Derivando $\mathcal{E}\left\{P(t) 1_{\{\theta(t)=j\}}\right\}$ e denotando $P_{j}(t)=\frac{d}{d t}\left(\mathcal{E}\left\{P(t) 1_{\{\theta(t)=j\}}\right\}\right), j \in \mathcal{S}$, obtemos:

$$
\begin{aligned}
\dot{P}_{j}(t, \Sigma)=( & \left.A_{j}-K_{j}(t) C_{j}\right) P_{j}(t, \Sigma)+P_{j}(t, \Sigma)\left(A_{j}-K_{j}(t) C_{j}\right)^{\prime}+\sum_{i=1}^{N} \lambda_{i j} P_{i}(t, \Sigma) \\
& +\left(B_{j} B_{j}^{\prime}+K_{j}(t) D_{j} D_{j}^{\prime} K_{j}(t)^{\prime}\right) p_{j}(t) .
\end{aligned}
$$

Para cada $\Sigma$ existe um $\epsilon_{j}>0$ suficientemente pequeno tal que as seguintes desigualdades são válidas: $\epsilon_{j} \mu_{j}<1$ e $\epsilon_{j} P_{j}\left(t^{*}, \Sigma\right) \leq I$; a última desigualdade 
nos permite escrever:

$$
\epsilon_{j} \mu_{j} P_{j}\left(t^{*}, \Sigma\right) \leq \mu_{j} I \leq Q_{j}\left(t^{*}\right)
$$

Agora, consideramos as funções $Z_{j}(t)=Q_{j}(t)-\epsilon_{j} \mu_{j} P_{j}(t, \Sigma)$ para cada $j \in \mathcal{S}$ e $t \geq t^{*}$ satisfazendo:

$$
\dot{Z}_{j}(t)=\mathcal{T}_{A-K(t) C, j}(Z(t))+\left(B_{j} B_{j}^{\prime}+K_{j}(t) D_{j} D_{j}^{\prime} K_{j}(t)^{\prime}\right)\left(1-\epsilon_{j} \mu_{j}\right) p_{j}(t)
$$

De (5.34) e $1-\epsilon_{j} \mu_{j}>0$, podemos verificar que a solução de (5.35) é definida positiva para $t \geq t^{*}$ e cada $j \in \mathcal{S}$. Este fato e a Hipótese 2 nos leva em $P_{j}(t) \leq P_{j}^{*}$, para $t \geq t^{*}$ e cada $j \in \mathcal{S}$, sendo $P_{j}^{*}=\left(\epsilon_{j} \mu_{j}\right)^{-1} Q^{*}$. Finalmente, temos $\mathcal{E}\{P(t)\}=\sum_{i \in \mathcal{S}} P_{i}(t) \leq \sum_{i \in \mathcal{S}} P_{i}^{*}=P^{*}$, concluindo a prova.

O resultado acima e a Proposição 11 levam no seguinte.

Corolário 6. Se a Hipótese 2 é satisfeita e $(A, B, \Lambda)$ is $A M$, então o estimador linear markoviano em (5.23) é estável.

Demonstração. A Proposição 12 mostra a positividade de $Q_{j}(t)$ para todo $t>0$ e $j \in \mathcal{S}$ sempre que $(A, B, \Lambda)$ seja AM. O resultado segue do Teorema 4.

Observação 5. É mostrado em (Costa et al., 2013) para um sistema detetável em média quadrática e estabilizável em média quadrática que $Q(t)$ converge e os ganhos associados dados por $K_{i}^{*}=\lim _{t \rightarrow \infty} K_{i}(t), i \in \mathcal{S}$, produzem uma dinâmica limite $A_{i}-K_{i}^{*} C_{i}$ estável em média quadrática, o qual pode ser usado para mostrar que o ELQMM é estável. Isto significa que a detetabilidade em média quadrática e a estabilizabilidade em média quadrática trabalham juntas como uma condição suficiente para estabilidade do filtro, em este sentido competindo com AM; de fato, existem sistemas que são AM e não estabilizável na média quadrática, veja Exemplo 6. Note que a prova do Teorema 4 é indiferente da dinâmica limite do sistema, o qual pode permitir futuras extensões. Como exemplo simples, podemos substituir as matrizes $C_{i}$ por matrizes periódicas $C_{i}(t+\delta)=C_{i}(t)$ (com período $\delta>1$ ), evitando assim a convergência de $Q(t)$, e ainda mantém o Teorema 4 inalterado.

Os exemplos a seguir consideram o ELQMM para ilustrar resultados obtidos nesta seção. Como já dissemos, este estimador está atraindo consideravelmente a atenção para aplicações na medida que ele é muito mais simples de implementar que o filtro de Kalman. 


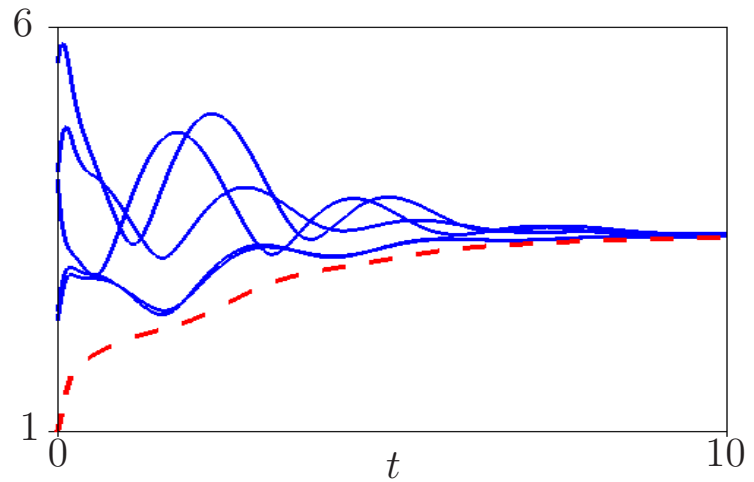

Figura 5.4: Norma das matrizes $\mathcal{E}\{P(t)\}$ para diferentes valores de $\Sigma$ (linhas sólidas) e $\mathcal{E}\{Q(t)\}$ (linha pontilhada) sobre o intervalo $[0,10]$ para o ELQMM do Exemplo 6.

Exemplo 6. Sejam $A$ e B como no Exemplo 5(a) e

$$
\begin{gathered}
C_{1}=\left(\begin{array}{ll}
1 & 1
\end{array}\right), \quad C_{2}=\left(\begin{array}{ll}
0 & -0.5
\end{array}\right), \quad C_{3}=\left(\begin{array}{ll}
1 & 0
\end{array}\right), \quad D_{1}=D_{2}=D_{3}=1, \\
\Lambda=\left(\begin{array}{ccc}
-1 & 0.5 & 0.5 \\
0.5 & -2 & 1.5 \\
0.01 & 0.99 & -1
\end{array}\right), \pi=1 / 3\left(\begin{array}{lll}
1 & 1 & 1
\end{array}\right), \bar{x}_{0}=\left(\begin{array}{c}
1.1 \\
-1.5
\end{array}\right) \quad e \Psi=I \in \mathfrak{R}^{2} .
\end{gathered}
$$

Primeiro consideramos $\Psi-\bar{x}_{0} \bar{x}_{0}^{\prime}$ como a matriz de covariância inicial para o projeto do ELQMM ("cenário nominal") e, em um segundo passo, usamos este filtro para estimar o estado de um sistema com covariância inicial $\Sigma$ ("cenário real"). Calculamos $Q_{i}(t)$ via (5.30) e verificamos a Hipótese 2 por inspeção direta do erro "nominal" $Q(t)=\sum_{i=1}^{N} Q_{i}(t)$. AM de $(A, B, \Lambda)$ foi verificada no Exemple 5, de tal maneira que o Teorema 4 garante que o filtro é estável. A Figura 5.4 ilustra a norma do erro "real" $P(t)$ para cinco diferentes condições iniciais $P(0)=\Sigma$, confirmando que $P(t)$ é limitada. Mencionamos em conexão com a Observação 5 que, ao aplicar o teste de inequações matriciais lineares que aparece em (Costa et al., 2013), o sistema é não estabilizável em média quadrática.

Exemplo 7. Considere o sistema $(A, B, C, D, \Lambda)$ com

$$
A_{1}=\left(\begin{array}{cc}
0 & 0 \\
0 & -1
\end{array}\right), \quad A_{2}=0, \quad A_{3}=I, \quad C_{1}=C_{3}=0, \quad C_{2}=\left(\begin{array}{cc}
0 & 0 \\
-10 & 0
\end{array}\right)
$$

$B_{j}=0 \in \mathfrak{R}^{2,3}, D_{j}=I \in \mathfrak{R}^{2}, j \in \mathcal{S}$, e $\Lambda, \pi$ e $\bar{x}_{0}$ são como no Exemplo 6 . 


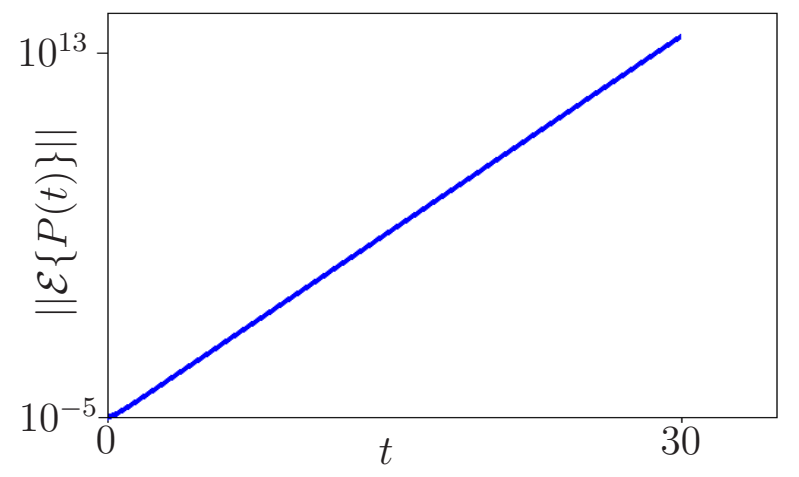

Figura 5.5: Norma da matriz $\mathcal{E}\{P(t)\}$ sobre o intervalo $[0,30]$ para o ELQMM do Exemplo $7($ a).

Aplicando o teste do posto no Teorema 1 verificamos que o sistema é não AM. $\mathrm{Na}$ sequência apresentamos os seguintes dois casos.

(a) $Q(0)=0 \in \mathfrak{R}^{2}$. O filtro possui erro nominal limitado $Q(t)=0$, enquanto o erro real $P(t)$ diverge exponencialmente como ilustrado na Figura 5.5, sendo que consideramos $\Sigma=10^{-6} I$.

(b) $Q(0)=I$. Notamos das simulações com $\Sigma=1.7$ I que tanto o erro nominal $Q(t)$ quanto o erro real $P(t)$ são limitados, veja Figura 5.6.

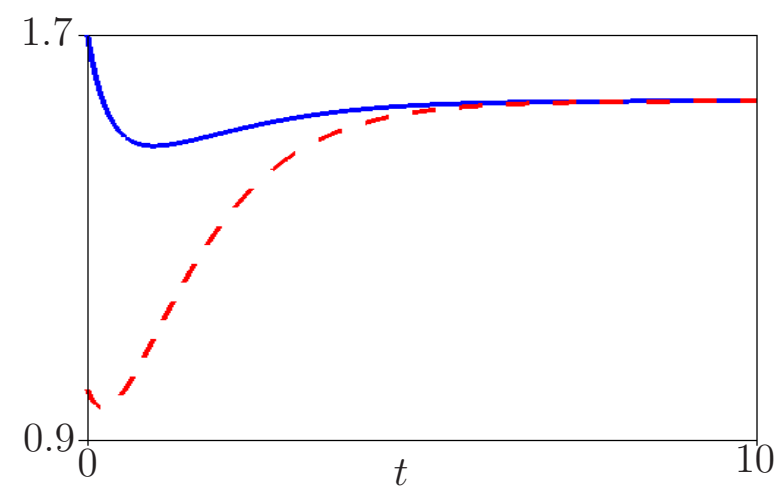

Figura 5.6: Norma da matriz $\mathcal{E}\{P(t)\}$ (linha sólida) e $\mathcal{E}\{Q(t)\}$ (linha pontilhada) sobre o intervalo $[0,10]$ para o ELQMM do Exemplo 7(b). 


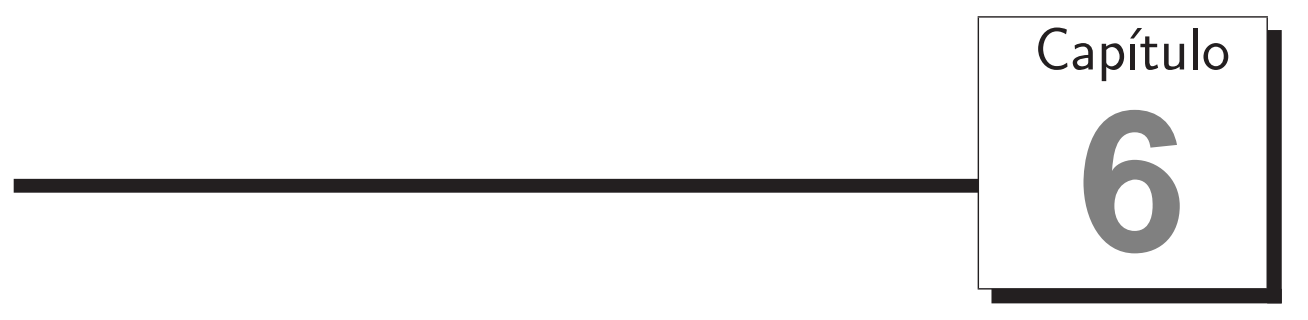

\section{Controlabilidade média e propriedades}

Neste capítulo estudamos a noção de controlabilidade média para SLSM. Embora seja possível estudar noções de $\pi$-CM , П-CM e CM análogas às de alcançabilidade, neste capítulo nos aprofundamos apenas na noção de CM, por brevidade. Esta noção requer que o valor esperado do gramiano correspondente, condicionado a cada estado inicial da cadeia, seja uma matriz definida positiva, independente da distribuição inicial $\pi$ (explicando por que aparece $\mathcal{S}$ ao invés de $S_{\pi}$ na definição abaixo); isto é, assumimos $\theta(0) \sim \pi$, com $\pi$ sendo uma distribuição inicial qualquer. Introduzimos um conjunto de matrizes e, baseado em certas propriedades de invariância, obtemos um teste que verifica o posto de tais matrizes, determinando se o sistema é CM ou não. Ilustramos esta condição através de exemplos numéricos.

Definição 12 (Controlabilidade média). Dizemos que $(A, B, \Lambda)$ é controlável em média se existe $t>0$ tal que $\mathcal{E}\left\{\Upsilon_{\mathrm{cnt}}(0, t) \mid \theta(0)=i\right\}>0$ para qualquer $i \in \mathcal{S}$.

Apresentamos um teste de posto para CM. Definimos $\mathbf{C} \in \mathcal{M}^{n, n \bar{N}}$ como:

$$
\mathbf{C}_{i}(A, B, \Lambda)=\left[\begin{array}{llll}
\mathcal{C}_{i}(0) & \mathcal{C}_{i}(1) & \ldots & \mathcal{C}_{i}(\bar{N}-1)
\end{array}\right]
$$

sendo $\mathcal{C}(k) \in \mathcal{M}^{n 0}$ como em (3.22). Dizemos que $\mathbf{C}$ é a coleção de matrizes de controlabilidade. 
Teorema 5. $(A, B, \Lambda)$ é $C M$ se e somente se $\operatorname{posto}\left(\mathbf{C}_{i}(A, B, \Lambda)\right)=n$, para todo $i \in \mathcal{S}$.

Demonstração. Dado $t>0$ qualquer, suponha que $\mathcal{E}\left\{\Upsilon_{\mathrm{cnt}}(0, t) \mid \theta(0)=i\right\}$ não é uma matriz definida positiva para algum $i \in \mathcal{S}$, de forma que, para um certo $v \neq 0$ temos $v^{\prime} \mathcal{E}\left\{\Upsilon_{\text {cnt }}(0, t) \mid \theta(0)=i\right\} v=0$. Pelo Lema 6 , isto é equivalente a $v^{\prime} \mathcal{C}_{i}(k) v=0, k \in \mathbb{Z}^{0}$, que por sua vez é equivalente a $\mathcal{C}_{i}(k) v=0, k \in \mathbb{Z}^{0} \mathrm{de}$ acordo com Corolário 4 , o que finalmente é equivalente a posto $\left(\mathbf{C}_{i}(A, B, \Lambda)\right)<$ $n$.

No seguinte exemplo mostraremos que a CM não é equivalente à AM apresentada no Capítulo 4. Com efeito, analogamente ao que ocorre em sistemas lineares a tempo discreto com saltos, controlabilidade não implica alcançabilidade, constituindo-se este fato aparentemente numa característica própria de sistemas com alguma variável discreta (aqui, o espaço de estados da cadeia é discreto).

Exemplo 8. Considere o sistema $(A, B, \Lambda)$ com

$$
\begin{gathered}
A_{1}=\left(\begin{array}{cc}
0 & -1 \\
1 & 0
\end{array}\right), A_{2}=0, A_{3}=\left(\begin{array}{ll}
1 & 1 \\
0 & 0
\end{array}\right), B_{1}=\left(\begin{array}{l}
1 \\
0
\end{array}\right), B_{2}=\left(\begin{array}{l}
0 \\
0
\end{array}\right) \\
B_{3}=\left(\begin{array}{ll}
0 & -1
\end{array}\right), \text { e } \Lambda=\left(\begin{array}{ccc}
-1 & 0 & 1 \\
0.5 & -1 & 0.5 \\
1 & 0 & -1
\end{array}\right) .
\end{gathered}
$$

Temos que, considerando qualquer distribuição inicial $\pi$, o sistema é CM pois aplicando o Teorema 5 temos que posto $\left(\mathbf{C}_{i}\right)=2$, para $i \in \mathcal{S}=\{1,2,3\}$. Note que a cadeia é tal que todos os estados tem probabilidade positiva de serem visitados, com número de blocos recorrentes $\bar{m}=1$. Assim, calculando $\mathbf{R}_{2}$ vemos que esta é a matriz nula com o que, usando o Teorema 2, o sistema é não $A M$.

Nossa experiência e os diversos exemplos com os quais lidamos sugerem que AM implica em CM. É interessante comentar que no cenário em que se considera uma distribuição inicial fixa, a noção de alcançabilidade não implica na controlabilidade como ocorre de forma geral em sistemas lineares. De fato, podemos definir analogamente à $\pi$-AM o conceito de $\pi$-CM. Isto é, dada uma distribuição inicial $\pi$, dizemos que o sistema $(A, B, \Lambda)$ é $\pi$-CM (controlável em média em relação a $\pi$ ) se existe $t>0$ tal que $\mathcal{E}\left\{\Upsilon_{\text {rch }}(0, t) \mid \theta(0)=i\right\}>0$, para todo $i \in \mathcal{S}_{\pi}$. O exemplo a seguir ilustra que $\pi$-AM não implica em $\pi$-CM. 
Exemplo 9. Seja $\pi=\left[\begin{array}{ll}1 / 2 & 1 / 2\end{array}\right]$ a distribuição inicial de uma cadeia com espaço de estados $\mathcal{S}=\{1,2\}$. Note de imediato que $\mathcal{S}_{\pi}=\mathcal{S}$. Considere agora o sistema $(A, B, \Lambda)$ dado por $A_{1}=1, A_{2}=0, B_{1}=1, B_{2}=0$, e a matriz de taxas dado por $\Lambda=\left(\begin{array}{cc}-1 & 1 \\ 0 & 0\end{array}\right)$. Aplicando o teste de $\pi$-AM (vide Teorema 1) temos que o sistema é $\pi$-AM. Por outro lado, considerando a definição proposta para $\pi$-CM vemos que $\mathcal{E}\left\{\Upsilon_{\mathrm{cnt}}(0, t) \mid \theta(0)=2\right\}=0$, para qualquer $t>0$, sendo o sistema não $\pi$-CM. Assim, $\pi$-AM não implica em $\pi$-CM.

No exemplo seguinte ilustramos que a CM é diferente de noções de controlabilidade para SLSM que aparecem na literatura, por exemplo a controlabilidade forte exposta em (Mariton, 1987).

Exemplo 10. Considere o sistema $(A, B, \Lambda)$ com

$$
A_{1}=A_{2}=I_{2}, \quad B_{1}=0, \quad B_{2}=I_{2}, \quad e \quad \Lambda=\left[\lambda_{i j}\right], i, j \in \mathcal{S}=\{1,2\} .
$$

(a). Suponha $\lambda_{11}=-\lambda_{12}=-\lambda_{21}=\lambda_{22}=-1$. É fácil verificar que $\left(A_{1}, B_{1}\right)$ é não controlável, porém, aplicando o teste no Teorema 5 podemos ver que as matrizes de controlabilidade $\mathbf{C}_{i}, i=1,2$, são de posto completo. Portanto $(A, B, \Lambda)$ é $C M$ a pesar da não controlabilidade dos sistemas em cada modo.

(b) Suponha $\lambda_{11}=\lambda_{12}=\lambda_{21}=\lambda_{22}=0$. Novamente usando Teorema 5 vemos que $\mathbf{C}_{1}$ é a matriz nula e portanto o sistema $(A, B, \Lambda)$ é não $C M$. Por outro lado, temos:

$$
\text { posto }\left[\begin{array}{llll}
B_{1} & A_{1} B_{1} & B_{2} & A_{2} B_{2}
\end{array}\right]=\text { posto }\left[\begin{array}{llllllll}
0 & 0 & 0 & 0 & 1 & 0 & 1 & 0 \\
0 & 0 & 0 & 0 & 0 & 1 & 0 & 1
\end{array}\right]=2 \text {. }
$$

Isto mostra que CM não implica no posto completo do empilhamento das matrizes de controlabilidade de cada modo.

\subsection{Factibilidade no problema de controlabilidade}

Vamos considerar a versão do SLSM dada por:

$$
\dot{x}(t)=A_{\theta(t)} x(t)+B_{\theta(t)} u(t)
$$

sendo $u(t) \in \mathbb{R}^{m}$ a entrada de controle. Daqui em diante, e no que resta deste capítulo, usaremos a notação $(A, B, \Lambda)$ para referir-nos ao sistema em (6.2). 
Mostraremos que CM é condição suficiente e necessária para a factibilidade do problema que definimos a seguir:

Problema de controlabilidade. Encontrar um processo de controle $u=$ $\{u(t)\}, \mathcal{G}_{t}$-adaptado, tal que $\mathcal{P}(x(t, x(0), u)=0)>0$.

Ou seja, mostramos que CM implica na existência de um controle $u$ que transfere qualquer $x(0) \in \mathbb{R}^{n}$ para $x(t)=0$, para algum $t \geq 0$, com probabilidade positiva; porém, não avançamos no projeto de tal controle.

Lema 10. Suponha que $(A, B, \Lambda)$ é $C M$. Então, o problema de controlabilidade é factivel

Demonstração. Suponha que para qualquer processo de entrada de controle $u$, $\mathcal{G}_{t}$-adaptado, e $t>0$, temos $\mathcal{P}\left(x\left(t, x_{0}, u\right)=0\right)=0$, para algum $(x(0), \theta(0))$. Então,

$$
x(t, x(0), u)=\Phi(t, 0) x(0)+\int_{0}^{t} \Phi(t, \tau) B_{\theta(\tau)} u(\tau) d \tau \neq 0, \quad \text { (q.c.) }
$$

ou, equivalentemente

$$
x(0) \neq-\int_{0}^{t} \Phi(0, \tau) B_{\theta(\tau)} u(\tau) d \tau, \quad \text { (q.c.). }
$$

Tomemos $u(t)=-B_{\theta(\tau)}^{\prime} \Phi(0, \tau)^{\prime} v$, para $v \in \mathbb{R}^{n}$ arbitrário; assim,

$$
\left.x(0) \neq\left(\int_{0}^{t} \Phi(0, \tau) B_{\theta(\tau)} B_{\theta(\tau)}^{\prime} \Phi(0, \tau)^{\prime} d \tau\right) v, \quad \text { (a.s. }\right) .
$$

Afirmamos que a dimensão do espaço nulo de $\Upsilon_{\text {cnt }}(0, t)$ é não nula (q.c.), portanto existe $\bar{v} \in \mathbb{R}^{n}, \bar{v} \neq 0$, tal que $\Upsilon_{\text {cnt }}(0, t) \bar{v}=0$, e pela sua vez temos $\bar{v}^{\prime} \mathcal{E}\left\{\Upsilon_{\mathrm{cnt}}(0, t) \mid \theta(0)=i\right\} \bar{v}=0$. O anterior nos permite dizer que $(A, B, \Lambda)$ é não CM.

Lema 11. Suponha que o problema de controlabilidade é factível. Então, $(A, B, \Lambda)$ é $C M$.

Demonstração. Se $(v, i)$ são tais que $v^{\prime} \mathcal{E}\left\{\Upsilon_{\text {cnt }}(0, t) \mid \theta(0)=i\right\} v=0$, então temos que $v^{\prime} \Upsilon_{\text {cnt }}(0, t) v=0$ (q.c.). Isto produz que o espaço nulo de $\Upsilon_{\text {cnt }}(0, t)$ seja não trivial (q.c.), e portanto o posto do gramiano de controlabilidade não inclua $\mathbb{R}^{n}$. Isto significa que existem condições iniciais $x_{0}$ tais que

$$
\int_{t_{0}}^{t_{1}} \Phi(0, \tau) B_{\theta(\tau)} u(\tau) d \tau \neq x_{0} \quad \text { (q.c.), }
$$


para qualquer processo de entrada de controle $\mathcal{G}_{t}$-adaptado; isto quer dizer que a trajetória contínua associada não pode ser direcionada para zero no intervalo finito $[0, t]$ (q.c.).

Exemplo 11. Considere sistema $(A, B, \Lambda)$ com

$$
A_{1}=\left[\begin{array}{ll}
1 & 1 \\
0 & 1
\end{array}\right], \quad B_{1}=\left[\begin{array}{ll}
1 & 0 \\
0 & 0
\end{array}\right], \quad A_{2}=0, \quad B_{2}=I_{2}, \quad \Lambda=\left[\begin{array}{cc}
0 & 0 \\
1 & -1
\end{array}\right]
$$

Consideremos a condição inicial $x_{0}=\left[\begin{array}{ll}0 & 1\end{array}\right]^{\prime}$ e $\theta(0)=1$. (3.22) produz

$$
\mathcal{C}_{1}(k)=\left[\begin{array}{cc}
c_{k} & 0 \\
0 & 0
\end{array}\right]
$$

$k=0,1, \ldots, 7$, sendo $c_{0}=1, c_{1}=3, c_{2}=7, c_{3}=15, c_{4}=31, c_{5}=63$, $c_{6}=127$, e $c_{7}=255$. Isto implica que posto $\left(\mathbf{C}_{1}\right)<2$ e conclui-se do teste do posto que o sistema é não $C M$. Note que $x_{0} \in \mathcal{N}\left(\mathcal{C}_{1}(k)\right)$, para $k=0,1, \ldots, 7$. É simples verificar que a segunda componente de $x(t)$ é dada por $x_{2}(t)=e^{t}$ (q.c.) para qualquer controle $u(t)$, confirmando que $x(0)$ não pode ser levado próximo de zero em um intervalo $[0, t]$ (veja Lema 11).

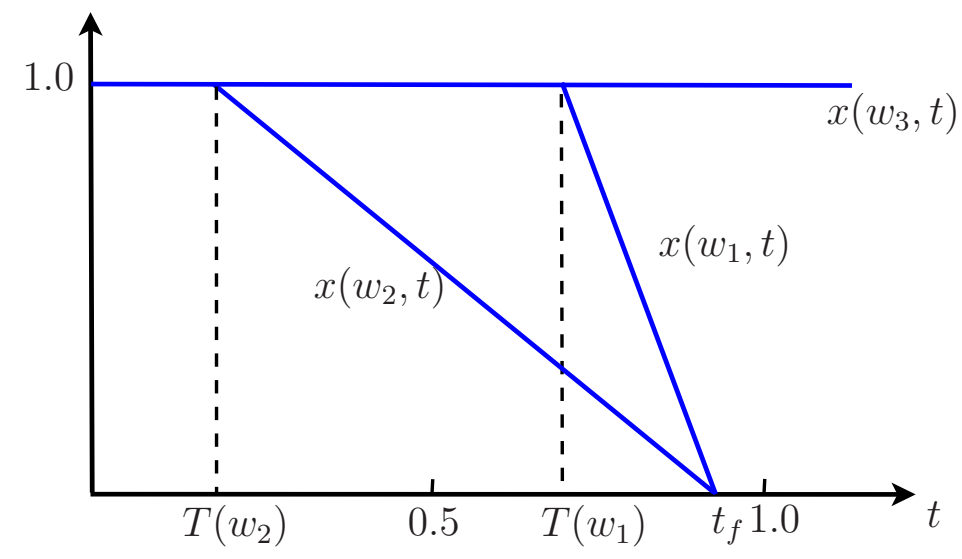

Figura 6.1: Três realizações diferentes de $x(t)$ para o sistema no Exemplo 12.

Exemplo 12. Considere o sistema $(A, B, \Lambda)$ com

$$
A_{1}=\left[\begin{array}{ll}
1 & 1 \\
0 & 0
\end{array}\right], \quad B_{1}=I_{2}, \quad A_{2}=B_{2}=0
$$


$x_{0}=\left[\begin{array}{ll}1 & 0\end{array}\right]^{\prime}, \theta(0)=2$ e $\Lambda$ como no Exemplo 11. Para este sistema verificamos que as matrizes de controlabilidade são de posto completo e portanto o sistema é CM. O controle u $(t)$ foi projetado como segue. Denotemos $T$ o instante de salto quando a cadeia de Markov chega em $\theta(t)=1$, e seja $u(t)=0$, para $t<T$, e, para $t \geq T$ seja $u(t)$ tal que, de alguma forma, $x(t)$ é enviado para zero no instante $t_{f}=0.9$ de forma linear. Ilustramos na Figura 6.1 três trajetórias $x\left(t, w_{\ell}\right), \ell=1,2,3$, correspondentes a três realizações diferentes $w_{1}, w_{2}$ e $w_{3}$ da cadeia de Markov. Podemos ver da figura que, $x\left(t, w_{3}\right)$ não alcança zero no intervalo $\left[0, t_{f}\right]$ nem mesmo para $T\left(w_{3}\right)>t_{f}$. 


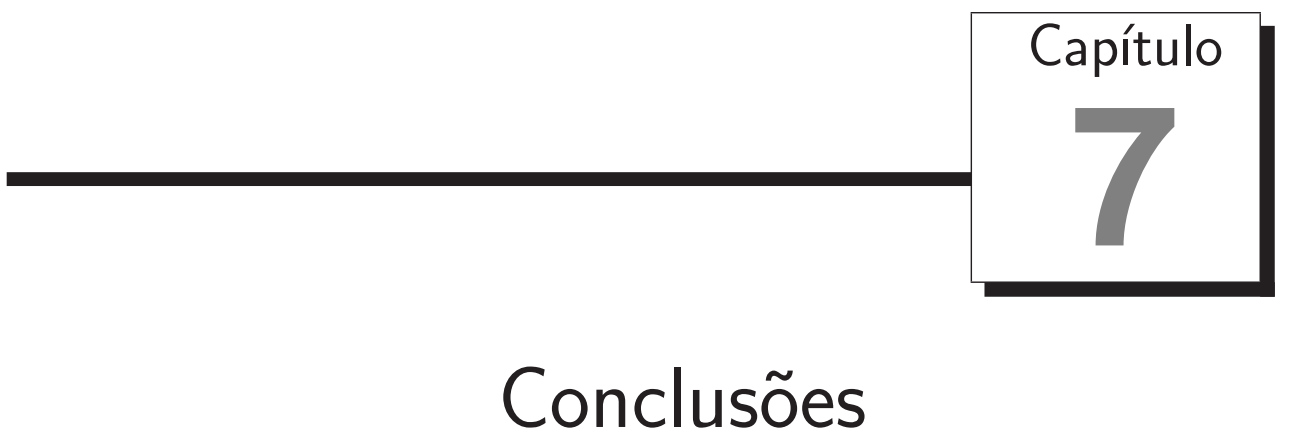

Neste trabalho estudamos oções estruturais de SLSM ligadas à esperança dos gramianos de alcançabilidade e controlabilidade para SLSM com ênfase na propriedade de que estes sejam definidos positivos. Os sistemas com a característica de que seus gramianos de alcançabilidade e controlabilidade sejam positivos em média foram denominados alcançáveis em média (AM) e controláveis em média $(\mathrm{CM})$, respectivamente. Introduzimos três noções de AM que dependem da distribuição de probabilidade inicial da cadeia de Markov: começando com a mais fraca, $\pi-\mathrm{AM}$, para situações em que $\pi$ é conhecido; logo segue a $\Pi-A M$, quando $\pi$ é tal que somente estados de Markov recorrentes tem probabilidade positiva de serem visitados; finalmente a mais forte, AM, quando não há informação disponível sobre $\pi$. Uma condição necessária e suficiente, testável computacionalmente, para a $\pi$-AM é obtida no Teorema 1, e sobre esta se baseia a derivação do teste computacional para AM, veja o Teorema 2.

Exploramos o uso da noção de AM na estrutura do sistema excitado aditivamente por um processo de Wiener visando a positividade do segundo momento do estado $E\left\{x(t) x(t)^{\prime}\right\}$ e do segundo momento condicional $X_{j}(t)$ para estados de Marvos recorrentes $j$. Uma dificuldade é que $X_{j}(t)$ é descrita pela equação diferencial não homogenia (5.3), envolvendo a distribuição $\pi(t)$ sem nenhum tipo de restrições sobre a cadeia de Markov, o que nos obriga a utilizar as matrizes $S_{j}(t)$, as quais, de certa forma, podem ser interpretadas como um segundo momento "modulado" (veja por favor a Observação 3), cumprindo o 
papel chave de ligar a definição de AM com o teste do posto do Teorema 1 e com a positividade de $X$ como estabelecido no Teorema 3. A positividade do segundo momento do estado de um SLSM não significa que o estado $x(t)$ atinja qualquer subconjunto do espaço $\mathbb{R}^{n}$ como ocorre nos sistemas lineares determinísticos (Davis, 1977); para uma ilustração, veja o Exemplo 3 o qual apresenta o comportamento da trajetória $x(t)$ de um sistema AM. Outro uso da AM é estudado no contexto de filtragem linear. Mostramos que a matriz de covariância do erro de estimação associado a filtros lineares, e em particular a lineares markovianos, é definida positiva com a interpretação de que a estimativa é "nunca precisa" no sentido das Proposições 11 e 12 respectivamente. No caso particular dos filtros lineares markovianos mostramos que AM também garante estabilidade deste tipo de filtro como apresentado no Corolário 6, com o significado de que a matriz de covariância do erro permanece limitada sob perturbações no valor inicial $\Psi$.

Quanto à CM obtivemos também um teste computacional que se baseia na verificação do posto das matrizes de controlabilidade. O conceito de CM independe da distribuição de probabilidade inicial e o teste é construído de maneira análoga ao teste da AM com ajuda das propriedades de invariância associadas ao gramiano de controlabilidade apresentadas na Seção 3. Os exemplos numéricos em relação a este conceito mostraram primeiramente que a CM não é equivalente a noções de controlabilidade presentes na literatura (vide Exemplo 10) e nem mesmo equivalente à $\mathrm{AM}$ como consta no Exemplo 8. Também apresentamos exemplos que ilustraram o fato de que CM é condição suficiente e necessária para factibilidade no problema de controlabilidade; isto é, vimos que, para sistemas $\mathrm{CM}$, uma entrada de controle $u(t)$ pode ser selecionada tal que $x\left(t_{f}\right)=0$ com probabilidade positiva; por outro lado, para sistemas que não são CM existem condições iniciais que não podem ser levadas para a origem (via qualquer entrada de controle $u$ ) sobre um intervalo finito $\left[0, t_{f}\right]$, quase certamente. Isto confirma a validade dos Lemas 10 e 11 respectivamente. 


\section{Apêndice A \\ Observabilidade e construtibilidade para sistemas lineares}

Em aplicações, o estado do sistema muitas vezes é necessitado mas não é acessível. Considere por exemplo o sistema invariante no tempo

$$
\dot{x}(t)=A x(t)+B u(t), \quad y(t)=C x(t)+D u(t) .
$$

Quando existe a inversa de $C$ podemos reconstruir instantaneamente o estado $x$ a partir da saída $y$ e a entrada $u$ achando a solução para $x$ na equação de saída $x(t)=C^{-1}(y(t)-D u(t))$. Porém, resulta obvio que isto só seria possível se o número de saídas fosse igual ao número de estados, ou seja, se $C$ for $n \times n$. Surge então a questão de se é possível em geral determinar o estado a traves da observação da resposta de um sistema a alguma entrada em um período de tempo. Uma resposta afirmativa a esta questão é fornecida se o sistema é observável. Observabilidade (Construtibilidade) tem a ver com a capacidade de determinar o estado atual $x\left(t_{0}\right)$ mediante o uso das saídas, $y(t)$, e entradas, $u(t), t \geq t_{0}\left(t \leq t_{0}\right)$, do sistema. Na continuação as seguintes definições.

Definição 13 (Observabilidade). O sistema (2.1) é observável em to, ou o par $(A(t), C(t))$ é observável em $t_{0}$, se o conjunto de estados $x$ tal que

$$
C(t) \Phi\left(t, t_{0}\right) x=0 \quad \text { para todo } t \geq t_{0},
$$


é igual ao conjunto $\{0\}$. Os estados $x$ satisfazendo (A.1) se chamam não observáveis.

Estados não observáveis não podem ser diferenciados do estado zero. Se na equação de saída em (2.1), usando (2.2), fizermos $\hat{y}(t)=y(t)-C(t) \int_{t_{0}}^{t} \Phi(t, \tau) B(\tau) u(\tau) d \tau+$ $D(t) u(t)$, obtemos $\hat{y}(t)=C(t) \Phi\left(t, t_{0}\right) x_{0}$ e portanto observamos que os estados não observáveis e o estado zero produzem a mesma saída.

Definição 14 (Construtibilidade). O sistema (2.1) é construível em $t_{1}$, ou o par $(A(t), C(t))$ é construível em $t_{1}$, se o conjunto de estados $x$ tal que

$$
C(t) \Phi\left(t, t_{1}\right) x=0 \quad \text { para todo } t \leq t_{1},
$$

é igual ao conjunto $\{0\}$. Os estados $x$ satisfazendo (A.2) se chamam não construíveis.

As seguintes matrizes são importantes nos conceitos de observabilidade e construtibilidade.

Definição 15. Definimos

(i) o gramiano observabilidade do sistema (2.1) como sendo a matriz $n \times n$

$$
\Gamma_{O}\left(t_{0}, t_{1}\right)=\int_{t_{0}}^{t_{1}} \Phi\left(\tau, t_{0}\right)^{\prime} C(\tau)^{\prime} C(\tau) \Phi\left(\tau, t_{0}\right) d \tau
$$

(ii) o gramiano de construtibilidade do sistema (2.1) como sendo a matriz $n \times n$

$$
\Gamma_{C}\left(t_{0}, t_{1}\right)=\int_{t_{0}}^{t_{1}} \Phi\left(\tau, t_{1}\right)^{\prime} C(\tau)^{\prime} C(\tau) \Phi\left(\tau, t_{1}\right) d \tau
$$

Proposição 13. O sistema (2.1) é observável em $t_{0}$ se e somente se existe $t_{1}>t_{0}$ tal que posto $\Gamma_{O}\left(t_{0}, t_{1}\right)=n$. Mais ainda, se o sistema é observável, o estado $x_{0}$ em $t_{0}$ é determinado por

$$
x_{0}=\left[\Gamma_{O}\left(t_{0}, t_{1}\right)\right]^{-1} \int_{t_{0}}^{t_{1}} \Phi\left(\tau, t_{0}\right)^{\prime} C(\tau)^{\prime} \hat{y}(\tau) d \tau .
$$

De maneira similar ao caso entre controlabilidade e alcançabilidade, existe também um resultado importante que relaciona a observabilidade e construtibilidade: Se o sistema (2.1) é observável em $t_{0}$, então é construível em algum $t_{1} \geq t_{0}$. Se $(2.1)$ é construível em $t_{1}$, então é observável em algum $t_{0} \leq t_{1}$. 
Mais uma vez, no caso de sistemas invariantes no tempo, considera-se $t_{0}=0$ e $t_{1}=T>0$ e, em analogia com o caso de alcançabilidade, é possível ver que o conjunto imagem de $\Gamma_{O}(0, T)$ é independente de $T$, para todo $T>0$ e além disso é igual ao conjunto imagem da matriz de observabilidade definida como

$$
\mathbb{O}(A, C)=\left[\begin{array}{llll}
C^{\prime} & A^{\prime} C^{\prime} & \cdots & \left(A^{n-1}\right)^{\prime} C^{\prime}
\end{array}\right]^{\prime} .
$$

Afirmamos que o sistema $\dot{x}(t)=A x(t)+B u(t), \quad y(t)=C x(t)+D u(t)$, ou o par $(A, C)$ é observável/construível se e somente se,

$$
\operatorname{posto} \mathcal{O}(A, C)=n
$$

Assim como no caso de controlabilidade, desde que a afirmação anterior é apenas uma propriedade das matrizes $A$ e $C$, ela pode ser considerada como definição para observabilidade. Para finalizar, temos um resultado de dualidade para sistemas lineares invariantes no tempo. Dizemos que, o sistema $\dot{x}(t)=A x(t)+B u(t), \quad y(t)=C x(t)+D u(t)$, ou o par $(A, B)$, é controlável se e somente se o sistema $\dot{x}(t)=A^{\prime} x(t)+C^{\prime} u(t), \quad y(t)=B^{\prime} x(t)+D^{\prime} u(t)$, ou o par $\left(A^{\prime}, B^{\prime}\right)$, é observável. Da mesma forma $(A, C)$ é observável se e somente se $\left(A^{\prime}, C^{\prime}\right)$ é controlável. 


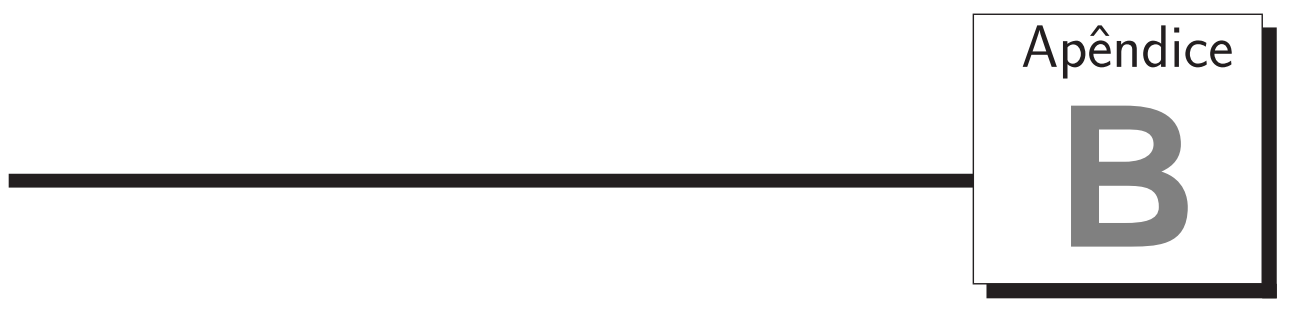

\section{Comparação de desempenho do ELQMM e o FKB para SLSM}

Vamos pôr em perspectiva o funcionamento do ELQMM comparando-o com o FKB a través do desenvolvimento de estudos de casos que ilustram as diferenças em suas concepções e desempenhos (um estudo mais detalhado pode ser encontrado em (Narváez e Costa, 2014) e em Narváez e Costa (2012) para uma versão a tempo discreto). Veremos que, quando a distribuição de probabilidade tende a ser "balanceada" (perto de ser equiprovável), e portanto toda realização da cadeia de Markov é menos predizível, o desempenho do FKB é melhor produzindo custos e erros de estimação menores que aqueles do ELQMM. Agora, a diferença de desempenho é menos marcante quando a distribuição de probabilidade em relação a um estado $i, \mathcal{P}(\theta(t)=i)$, permanece próxima da unidade por um longo período de tempo. De fato, os desempenhos dos filtros são similares quando a distribuição tende a ser "concentrada" em algum estado.

Consideremos a seguinte versão do SLSM em (2.8):

$$
\begin{aligned}
& d x(t)=(A(t, \theta(t)) x(t)+\bar{B}(t, \theta(t)) u(t)) d t+B(t, \theta(t)) d w(t) \\
& d y(t)=C(t, \theta(t)) x(t) d t+D(t, \theta(t)) d v(t),
\end{aligned}
$$

sendo que agora é introduzido o processo entrada $u(t) \in \mathbb{R}^{m}$. Considere o 
custo quadrático,

$$
\begin{aligned}
J(x(0), \theta(0), u)= & \mathcal{E}\left\{\int_{0}^{T}\left[x(t)^{\prime} F_{\theta(t)}(t) x(t)+u(t)^{\prime} G_{\theta(t)}(t) u(t)\right] d t\right. \\
& \left.+x(T)^{\prime} F_{\theta(T)}(T) x(T)\right\},
\end{aligned}
$$

sendo $T$ um número real positivo $(T>0)$ e $F_{\theta(t)}(t) \geq 0, G_{\theta(t)}(t)>0$, para cada $t \geq 0$. O problema de controle ótimo quadrático com horizonte finito para SLSM a tempo contínuo consiste em encontrar uma política de controle pertencendo à família de funções factíveis $u(t)=\mu(t, y(s), \theta(s), 0 \leq s \leq t)$, $0 \leq t \leq T$, mensuráveis sobre $\left(\mathcal{F}_{t}, \mathcal{G}_{t}\right)$, que minimiza (B.2) sujeito to (B.1); o valor mínimo de (B.2) é denotado aqui por $J^{*}$. Devido à indisponibilidade do estado $x(t)$, a solução deste problema é abordada construindo um estimador linear (ou observador, veja (Kwakernaak e Sivan, 1972)) dado como:

$$
\begin{aligned}
d \hat{x}(t) & =\left(A_{\theta(t)}(t) \hat{x}(t)+\bar{B}_{\theta(t)}(t) u(t)\right) d t+K(t)\left(d y(t)-C_{\theta(t)}(t) \hat{x}(t) d t\right), \\
\hat{x}(0) & =\hat{x}_{0}
\end{aligned}
$$

o qual se torna ótimo entre todos os estimadores lineares ao encontrar valores $\hat{x}_{0}$ e $K(t)$ apropriados tais que o erro de estimação associado é minimizado para cada $t \geq 0$. O estimador linear ótimo é interconectado com uma lei ótima de controle dada em forma linear como:

$$
u(t)=H(t) \hat{x}(t),
$$

e, então, ao substituir (B.4) em (B.3), obtemos o controlador de malha fechada:

$$
\begin{aligned}
d \hat{x}(t) & =\left(A_{\theta(t)}(t)+\bar{B}_{\theta(t)}(t) H(t)-K(t) C_{\theta(t)}(t)\right) \hat{x}(t) d t+K(t) d y(t), \\
u(t) & =H(t) \hat{x}(t) .
\end{aligned}
$$

O filtro para estimar $x$ e a lei de controle para minimizar o custo como se $x$ for observada completamente, são construídos separadamente e logo combinadas substituindo $x$ na lei de controle pela estimativa. Este procedimento leva na solução ótima em situações onde o Princípio de Separação (SP) é válido, mas também é comumente usado independentemente de verificar o cumprimento do PS e bons resultados são reportados frequentemente. As diferenças entre o ELQMM e o FKB não são muito discutidas e exploradas, por exemplo não é muito disseminado que a única distinção entre eles é que o ELQMM 
foi projetado atendendo uma "restrição markoviana". Enquanto que a função matricial $K(t)$ dada em (B.3) não só depende do parâmetro tempo $t$ mas também de valores da cadeia de Markov $\theta(s)$, para $0 \leq s \leq t$, o qual evita que a propriedade markoviana seja válida para o FKB. Em contraste, a matriz de ganho do ELQMM é definida como uma função determinística com valores $K(t, \theta(t)):=K_{\theta(t)}(t)$. Quanto ao ganho ótimo de controle, ele é dado por uma matriz $H(t, \theta(t)):=H_{\theta(t)}(t)$, sendo este o ganho ótimo do controle para o problema com informação completa do estado e portanto indiferente de como será estimado $x$. O ganho ótimo de controle pode ser calculado offline de forma reversa no tempo a partir de $t=T$ até $t=0$, para cada $i \in \mathfrak{S}$, veja por exemplo (Ji e Chizeck, 1992; Costa et al., 2013); quando este controle é implementado no problema com informação parcial do estado, usamos o PS e substituímos $x(t)$ no controle linear de retroalimentação do estado por uma estimativa $\hat{x}(t)$, isto é, fazemos $u(t)=H_{\theta(t)}(t) \hat{x}(t)$. As estimativas $\hat{x}(t)$ do estado são obtidas resolvendo problemas de otimização que envolvem a minimização de $\|e(t)\|$, sendo $e(t)=x(t)-\hat{x}(t)$ o erro de estimação associado à estimativa $\hat{x}(t)$. O problema de otimização pode incorporar restrições que tornam mais fácil a busca da solução, ou reduzem a complexidade do filtro, eventualmente deteriorando a qualidade da estimativa. Na continuação veremos que o FKB requer linearidade enquanto que o ELQMM requer tanto linearidade quanto "markovianidade".

\section{Filtro de Kalman-Bucy}

Desde que há observação completa de $\theta$, a cada instante $t>0$ os valores $\theta(s), 0 \leq s \leq t$ são conhecidos e (B.1) se torna um sistema linear variante no tempo. Neste caso o FKB, denotado por $\hat{x}^{*}$, é o estimador linear ótimo para o SLSM dado por:

$$
d \hat{x}^{*}(t)=\left(A_{\theta(t)}(t) \hat{x}^{*}(t)+\bar{B}_{\theta(t)}(t) u(t)\right) d t+K^{*}(t)\left(d y(t)-C_{\theta(t)}(t) \hat{x}^{*}(t) d t\right),
$$

com condição inicial $\hat{x}^{*}(0)=\bar{x}_{0}$ e matriz ganho $K^{*}(t)$ dada por $K^{*}(t)=$ $Q^{*}(t) C_{\theta(t)}(t)^{\prime}\left(D_{\theta(t)}(t) D_{\theta(t)}(t)\right)^{-1}$, sendo que para cada realização dada do pro- 
cesso de salto, $Q^{*}(t)$ satisfaz a equação matricial diferencial:

$$
\begin{aligned}
\dot{Q}^{*}(t) & =A_{\theta(t)}(t) Q^{*}(t)+Q^{*}(t) A_{\theta(t)}(t)^{\prime}+B_{\theta(t)}(t) B_{\theta(t)}(t)^{\prime}-Q^{*}(t) C_{\theta(t)}(t)^{\prime} \\
& \times\left(D_{\theta(t)}(t) D_{\theta(t)}(t)^{\prime}\right)^{-1} C_{\theta(t)}(t) Q^{*}(t)
\end{aligned}
$$

com condição inicial $Q^{*}(0)=\Psi-\bar{x}_{0} \bar{x}_{0}^{\prime} \cdot Q^{*}(t)$ determina o segundo momento condicional dado como $\mathcal{E}\left\{e^{*}(t) e^{*}(t)^{\prime} \mid \mathcal{G}_{t}\right\}$, sendo $e^{*}(t)$ o erro de estimação associado ao FKB. Como $K^{*}(t)$ e $Q^{*}(t)$ dependem das realizações do processo $\theta(t)$ (portanto ambos são processos estocásticos), pré-computar os ganhos do FKB é em alguns casos inviáveis. Por último, vale a pena notar que quando $x(0)$ é assumida Gaussiana o FKB coincide com a esperança condicional $\mathcal{E}\left\{x(t) \mid \mathcal{F}_{t}, \mathcal{G}_{t}\right\}$ a qual não é só o melhor estimador linear mas o melhor entre os estimadores que podem ser obtidos.

\section{Esimador Linear Quadrático Médio Mínimo}

O ELQMM, denotado por $\hat{x}$, é um estimador linear caracterizado pela vantagem computacional em relação ao FKB no sentido que suas matrizes de ganho podem ser calculadas offline. O problema de filtragem ótima do qual se obtém este estimador introduz duas restrições importantes, relativamente fortes - o filtro tem de ser linear e markoviano, apresentando uma certa estrutura predeterminada. Obtém-se um conjunto de matrizes de ganho das quais o $i$-ésimo ganho é selecionado no tempo $t$ quando $\theta(t)=i$, enquanto a operação do sistema está em andamento. O ELQMM comporta-se como um sistema linear markoviano dado por:

$$
d \hat{x}(t)=\left(A_{\theta(t)}(t) \hat{x}(t)+\bar{B}_{\theta(t)}(t) u(t)\right) d t+K_{\theta(t)}(t)\left(d y(t)-C_{\theta(t)}(t) \hat{x}(t) d t\right),
$$

com condição inicial $\hat{x}(0)=\bar{x}_{0}$ e matriz de ganho $K_{j}(t)$ definida como:

$$
K_{j}(t)=Q_{j}(t) C_{j}(t)^{\prime}\left(D_{j}(t) D_{j}(t) p_{j}(t)\right)^{-1}
$$

sendo, para cada $j \in \mathcal{S}, Q_{j}(t)$ satisfaz a equação diferencial matricial acoplada:

$$
\begin{aligned}
\dot{Q}_{j}(t)= & A_{j}(t) Q_{j}(t)+Q_{j}(t) A_{j}(t)^{\prime}+\sum_{i=1}^{N} \lambda_{i j} Q_{i}(t)+B_{j}(t) B_{j}(t)^{\prime} p_{j}(t) \\
& \times-Q_{j}(t) C_{j}(t)^{\prime}\left(D_{j}(t) D_{j}(t)^{\prime} p_{j}(t)\right)^{-1} C_{j}(t) Q_{j}(t), \\
Q_{j}(0)= & \left(\Psi-\bar{x}_{0} \bar{x}_{0}{ }^{\prime}\right) p_{j}(0) .
\end{aligned}
$$


A solução de (B.10) é tal que $Q_{j}(t)=\mathcal{E}\left\{e(t) e(t)^{\prime} 1_{\{\theta(t)=j\}}\right\}$, sendo $e(t)$ o erro de estimação associado ao ELQMM. O grau de subotimalidade é fortemente associado com a conjuntura de que o cálculo dos filtros do ELQMM em B.9 e B.10 não usam toda a informação disponível trazida em $\left(\mathcal{F}_{t}, \mathcal{G}_{t}\right)$ mas somente a distribuição "não filtrada" $p_{j}(t)=\mathcal{P}(\theta(t)=j)$, a qual apenas depende de $p(0)$.

Observação 6. Como dissemos antes, a diferença mais significante entre o FKB e o ELQMM é que este último é markoviano. Para obter esta característica, o cálculo dos ganhos $K_{i}(t)$ desconsidera a informação $\theta(s), 0 \leq s \leq t$ que está disponível no tempo t, a qual é a "fonte de subotimalidade" básica em relação ao FKB; note em esta conexão que a distribuição $p(t)$ aparecendo na equação de Riccati em (B.10) é indiferente das observações. O lado positivo disto é que é possível o cálculo offline dos ganhos, uma característica muito valiosa para a implementação do filtro. Em resumo, o ELQMM perde informação para ganhar simplicidade. Assim, $p(t)$ é a chave para entender as principais diferenças no desempenho dos filtros; quanto mais concentrada estiver $p(t)$ em um estado $i$ durante um intervalo de tempo mais tempo permanece a trajetória $\theta(t)$ no estado $i$ no mesmo intervalo, e mais parecidos os desempenhos dos filtros são. No outro extremo, quantos mais balanceada a distribuição $p(t)$ menos predizivel as realizações da cadeia fazendo com que maior seja a diferença no desempenho dos filtros.

\section{Testes numéricos}

Em esta seção apresentamos estudos de caso que envolvem situação nas que a diferença de desempenho entre os filtros é grande/pequena. Os custos incorridos pelos controles e filtros combinados são estimados usando simulação Monte Carlo baseada em 10,000 realizações. Para ilustrar a efetividade de cada filtro empregamos a integral do erro quadrático médio no horizonte $T$.

Exemplo 13. Considere o SLSM em (B.1) com matrizes:

$$
\begin{gathered}
A_{1}=\left[\begin{array}{cc}
-1 & 0.05 \\
10 & -1
\end{array}\right], A_{2}=\left[\begin{array}{cc}
1 & -0.9 \\
1.1 & 0.6
\end{array}\right], A_{3}=\left[\begin{array}{cc}
0 & -1.7 \\
1.4 & -0.5
\end{array}\right], \bar{B}_{1}=\left[\begin{array}{l}
1 \\
0
\end{array}\right], \\
\bar{B}_{2}=\left[\begin{array}{l}
0 \\
0
\end{array}\right], \bar{B}_{3}=\left[\begin{array}{c}
0 \\
-0.5
\end{array}\right], B_{1}=\left[\begin{array}{ccc}
1 & 0.2 & -1.9 \\
-0.1 & 1.4 & -0.3
\end{array}\right], B_{2}=\left[\begin{array}{ccc}
0.4 & 0.3 & 0.8 \\
0.9 & 0 & 0.1
\end{array}\right],
\end{gathered}
$$




$$
\begin{aligned}
& B_{3}=\left[\begin{array}{ccc}
0 & -0.9 & 0 \\
1.2 & -0.2 & 0.1
\end{array}\right], C_{1}=\left[\begin{array}{cc}
-0.3 & 0.3 \\
0.9 & -0.7
\end{array}\right], C_{2}=\left[\begin{array}{ll}
0.9 & 0.3 \\
0.1 & 2.5
\end{array}\right], \\
& C_{3}=\left[\begin{array}{cc}
-1 & 0.5 \\
0 & -1.2
\end{array}\right], D_{i}=I, i=1, \ldots, 3, F_{1}=I, F_{2}=2 F_{1}, F_{3}=0, e,
\end{aligned}
$$

$G_{1}=10, G_{2}=0.5$, e $G_{3}=1$, sendo $I$ a matriz identidade de dimensão apropriada. Seja $x(0)$ com distribuição Gaussiana e assuma também que $p_{j}(0)>0$ para todo $j \in \mathcal{S}$. Finalmente, a matriz de taxas de probabilidades de transição é dada por:

$$
\Lambda=\left[\begin{array}{ccc}
-2 & 1 & 1 \\
1 & -3 & 2 \\
1.5 & 0.5 & -2
\end{array}\right]
$$

Os custos incorridos pelos FKB/controle ótimo e ELQMM/controle ótimo foram estimados por simulação Monte Carlo e são apresentados na seguinte tabela.

\section{Valores de $J^{*}$ usando KBF e ELQMM}

\begin{tabular}{|ccc|}
\hline & $J^{*}-\boldsymbol{F K B}$ & $J^{*}-\boldsymbol{E L Q M M}$ \\
\hline$T=1:$ & $11,941.083$ & 12,132.159 \\
\hline$T=2:$ & $53,754.037$ & $56,458.934$ \\
\hline$T=3:$ & 212,982.22 & 223,101.63 \\
\hline
\end{tabular}

A tabela seguinte confirma que o FKB é o melhor estimador linear do vetor estado $x(t)$ no sentido que este filtro minimiza a função $\ell=\int_{0}^{T} \mathcal{E}\left\{\|e(t)\|^{2}\right\} d t$. Observe que os custos e os valores do erro de estimação atingidos pelos FKB são menores que aqueles do ELQMM, confirmando a subotimalidade descrita antes.

Valores de $\ell$ usando FKB e ELQMM

\begin{tabular}{|ccc|}
\hline & $\ell-\boldsymbol{K B F}$ & $\ell-\boldsymbol{F} \boldsymbol{C F}$ \\
\hline$T=1:$ & $2,794.7941$ & $3,320.6918$ \\
\hline$T=2:$ & $5,260.8757$ & $6,483.0149$ \\
\hline$T=3:$ & $8,847.1907$ & $10,756.499$ \\
\hline
\end{tabular}

Exemplo 14. Considere o SLSM do Exemplo 13 com

$$
\Lambda=\left[\begin{array}{ccc}
-10 & 10 & 0 \\
15 & -20 & 5 \\
10^{-2} & 0 & -10^{-2}
\end{array}\right]
$$


sendo que $\theta=3$ é um estado markoviano com transição bastante lenta, o que produz $\lim _{t \rightarrow \infty} p_{3}(t) \approx 1$. Tipicamente, em esta situação e para $t$ suficientemente grande, obtemos que $Q_{j}(t) \approx 0$ para cada $j \neq 3$ (veja (B.10)) e, por outro lado a maioria das realizações da cadeia de Markov permanece em $\theta(t)=3$, para longos períodos de tempo t. Isto faz que $Q^{*}(t)$ (dado por (B.7)) se aproxime de $Q_{3}(t)$ e os desempenhos dos filtros sejam similares após um tempo transiente. De fato, obtivemos $J^{\star}-F K B=16,653.748$ e $J^{\star}-E L Q M M=16,662.768$, $\ell-F K B=4,364.098$ e $\ell-E L Q M M=4,585.139$ considerando horizonte de tempo $T=5$.

Exemplo 15. Consideramos 1,000 SLSM com $n=2$ e $N \in\{1,2\}$. Em cada sistema geramos aleatoriamente (usando o comando "rand" do Scilab (Scilab Enterprises, 2012)) as coleções de matrizes $A, \bar{B}, C, D, B, F, G$. A matriz de taxas é fixa, dada por:

$$
\Lambda=\left[\begin{array}{cc}
-1 & 1 \\
1 & -1
\end{array}\right] .
$$

Para marcar as diferenças nos desempenhos dos filtros, consideramos a quantidade $\mathcal{R}=J^{\star}-F K B / J^{\star}-E L Q M M$ para cada sistema e apresentamos Tabela 15 a média $\overline{\mathcal{R}}$ para diferentes horizontes de tempo, ilustrando o desempenho significativamente melhor do FKB para horizontes grandes de tempo. Em $90 \%$ dos sistemas observamos que o custo atingido usando o FKB é pelo menos $10 \%$ menor quando comparado com o ELQMM. A distribuição ao longo do tempo é equiprovável sendo esta situação a mais desfavorável para o ELQMM como indicado na Observação 6.

Razão média entre os valores $J^{\star}-F K B$ e $J^{\star}$-ELQMM

\begin{tabular}{|cc|}
\hline & $\overline{\mathcal{R}}$ \\
\hline$T=1:$ & 0.9754584 \\
\hline$T=2:$ & 0.7865339 \\
\hline$T=3:$ & 0.5533218 \\
\hline
\end{tabular}

Os anteriores exemplos numéricos ilustraram que os custos incorridos pelo FKB são menores que aqueles do ELQMM, sugerindo pela sua vez que a diferença no desempenho está em proporção direta com o horizonte $T$, porém a diferença é menos marcante quando a distribuição de probabilidade cadeia de Markov tende a ser concentrada para longos períodos de tempo.

Colocando estes filtros em perspectiva fica claro do por quê eles são diferentes, o qual nos ajuda a escolher qual filtro usar em aplicações do mundo real. 
Sistemas caracterizados por estados markovianos que tendem a ser absorventes ou lentos (juntamente com os rápidos) em geral apresentam bom desempenho com o ELQMM, enquanto que o FKB é preferível, dependendo da viabilidade de implementação, para sistemas com distribuições balanceadas. Futuramente projetamos uma proposta de filtro híbrido que combine ambos o FKB e o ELQMM, tendo em conta alguma medida de quão concentrada a distribuição de probabilidade é em cada instante tempo. 


\section{Referências Bibliográficas}

Anderson, B. D. O. Stability properties of kalman-bucy filters. Journal of the Franklin Institute, v. 291, p. 137-144, 1971.

Antsaklis, P. J.; Michel, A. N. Linear systems. Birkhauser, 2006.

Bhattacharya, R. B.; Waymire, E. C. Stochastic processes with aplications. Wiley-Interscience, 1990.

Bujorianu, M. L.; Lygeros, J. New insights on stochastic reachability. In: Proc. CDC'07 46th IEEE Conference on Decision and Control, New Orleans, LA, USA, 2007.

Costa, E. F.; Astolfi, A. Characterization of exponential divergence of the kalman filter for time-varying systems. SIAM Journal on Control and Optimization, v. 48, p. 2917-2944, 2009.

Costa, E. F.; Do VAL, J. B. R. On the detectability and observability of continuous-time Markov jump linear systems. SIAM Journal on Control and Optimization, v. 41, n. 4, p. 1295-1314, 2002a.

Costa, E. F.; Do VAL, J. B. R. Weak detectability and the linear-quadratic control problem of discrete-time Markov jump linear systems. International Journal of Control, Special Issue on Switched and Polytopic Linear Systems, v. 75 , n. 16/17, p. 1282-1292, 2002 b.

Costa, E. F.; Do Val, J. B. R.; Fragoso, M. D. A new approach to detectability of discrete-time infinite Markov jump linear systems. SIAM Journal on Control and Optimization, v. 43, p. 2132-2156, 2005a. 
Costa, E. F.; Manfrim, A. L. P.; do Val, J. B. R. Weak controllability and weak stabilizability concepts for linear systems with Markov jump parameters. In: Proc. ACC'06 American Control Conference, Minneapolis,USA, 2006.

Costa, O. L. V.; do Val, J. B. R.; Geromel, J. C. Continuoustime state-feedback $H_{2}$-control of markovian jump linear systems via convex analysis. Automatica, v. 35, p. 259-268, 1999.

Costa, O. L. V.; Fragoso, M. Discrete-time LQ-optimal control problems for infinite Markov jump parameter systems. IEEE Transactions on Automatic Control, v. AC-40, p. 2076-2088, 1995.

Costa, O. L. V.; Fragoso, M. D.; Marques, R. P. Discrete-time markovian jump linear systems. New York: Springer-Verlag, 2005b.

Costa, O. L. V.; Fragoso, M. D.; Todorov, M. G. Continuous-time markovian jump linear systems. New York: Springer-Verlag, 2013.

Costa, O. L. V.; Marques, R. Robust $H_{2}$-control for discrete-time markovian jump linear systems. International Journal of Control, v. 73, n. 1, p. $11-21,2000$.

DAvis, J. H. Foundations of deterministic and stochastic control. Birkhauser, 2002.

Davis, M. H. A. Linear estimation and stochastic control. Chapman and Hall, 1977.

Do VAL, J.; Costa, E. F. Numerical solution for the linear-quadratic control problem of Markov jump linear systems and a weak detectability concept. Journal of Optimization Theory and Applications, v. 114, n. 1, p. 69-96, 2002.

Do VAl, J. B. R.; Costa, E. F. Stabilizability and positiveness of solutions of the jump linear quadratic problem and the coupled algebraic Riccati equation. IEEE Transactions on Automatic Control, v. 50, n. 5, p. 691-695, 2005.

Dragan, V.; Morozan, T.; Stoica, A. M. Mathematical methods in robust control of linear stochastic systems. 2nd ed. Springer, 2013. 
Fragoso, M.; Baczynski, J. Optimal control for continuous time LQ problems with infinite Markov jump parameters. SIAM Journal on Control and Optimization, v. 40, n. 1, p. 270- 297, 2001.

Fragoso, M. D.; Costa, O. L. V. A unified approach for stochastic and mean square stability of continuous-time linear systems with markovian jumping parameters and additive disturbances. SIAM journal on control and optimization, ISSN 0363-0129, v. 4, p. 1165-1191, 2005.

Fragoso, M. D.; Costa, O. L. V. A separation principle for the continuous-time lq-problem with markovian jump parameters. IEEE Transactions on Automatic Control, v. 55, n. 12, p. 2692-2707, 2010.

Gomes, M. J. F.; Costa, E. F. On the stability of the recursive kalman filter with markov jump parameters. In: Proc. ACC'10 American Control Conference, Baltimore,USA, 2010.

JazWinski, A. H. Stochastic processes and filtering theory. Academic Press, 1970.

Ji, Y.; ChizeCK, H. J. Controlability, stabilizability and continuous time Markovian jump linear quadratic control. IEEE Transactions on Automatic Control, v. 35, n. 7, p. 777-788, 1990.

Ji, Y.; ChIzeCK, H. J. Jump linear quadratic gaussian conrol in continuous time. IEEE Transactions on Automatic Control, v. 37, n. 12, p. 1884-1892, 1992.

KWAKERnAAK, H.; Sivan, R. Linear optimal control systems. WileyInterscience, 1972.

Mariton, M. Stochastic controllability of linear systems with markovian jumps. Automatica, v. 23, n. 6, p. 783-785, 1987.

Morozan, T. Stability and control for linear systems with jump Markov perturbations. Stochastic Analysis and Applications, v. 13, n. 1, p. 91-110, 1995.

Narváez, A. R. R.; Costa, E. F. On the observability of continuos time linear systems with Markov jump parameters. In: Proc. ACC'10 American Control Conference, Baltimore, MD, USA, 2010. 
Narváez, A. R. R.; Costa, E. F. On the controllability of continuoustime markov jump linear systems. In: Proc. IFAC 18th World Congress of the International Federation of Automatic Control, Milano, Italy, 2011.

Narváez, A. R. R.; Costa, E. F. A comparative study between the kalman filter and a linear markovian filter for markov jump linear systems. In: Ponencias del XV Congreso Latinoamericano de Control Automático, Lima, Perú, 2012.

Narváez, A. R. R.; Costa, E. F. A comparative case study between the kalman-bucy filter and a linear markovian filter for continuous-time markov jump linear systems. In: Memorias del XVI Congreso Latinoamericano de Control Automático, Cancún, Quintana Roo, México, 2014.

Nishimura, T. Error bounds of continuous kalman filters and the application to orbit determination problems. IEEE Transactions on Automatic Control, v. AC-12, p. 268-275, 1967.

NorRIs, J. R. Markov chains. Cambridge University Press, 1998.

Sangsuk-IAM, S.; Bullock, T. E. Analysis of discrete-time Kalman filtering under incorrect noise covariances. IEEE Transactions on Automatic Control, v. 35, n. 12, p. 1304-1309, 1990.

ScIlab ENTERPRISES Scilab: Free and open source software for numerical computation. Scilab Enterprises, Orsay, France, 2012.

Disponível em http://www.scilab.org

Sun, Z.; Ge, S. S. Switched linear systems control and design. Springer, 2005.

ZABCZYK, J. Controllability of stochastic linear systems. Systems \& Control Letters, v. 1, p. 25-31, 1981.

Zakai, M.; Snyders, J. Stationary probability measures for linear differential equations driven by white noise. Journal of differential equations, v. 8, p. 27-33, 1970. 